\title{
On the Highly Unsteady Dynamics of Multiple Thermal Buoyant Jets in Cross Flows
}

\author{
Marcello Lappa ${ }^{1}$ \\ ${ }^{1}$ Department of Mechanical and Aerospace Engineering, University of Strathclyde, James Weir \\ Building, 75 Montrose Street, Glasgow, G1 1XJ, UK - email: marcello.lappa@strath.ac.uk, \\ marlappa@unina.it
}

\begin{abstract}
Thermal plumes of small scale generated by spatially separated heat sources can form, like atoms in a chemical compound, complex structures of different kind and with distinct behaviors. The situation becomes even more complex if plumes can interact with imposed vertical shear (a horizontal wind). In this analysis a 'minimal framework' based on the application of a filtering process to the governing balance equations for mass, momentum and energy (falling under the general heading of 'Large Eddy Simulation' approach), is used together with Direct Numerical Simulation to inquiry about the relative importance of buoyancy and vertical shear effects in determining the patterning scenario when highly unsteady dynamics are established (turbulent flow). Emerging patterns range from the flow dominated by a static rising jet produced by the aggregation of plumes, which are pushed by horizontal leftwards and rightwards winds towards the center of the physical domain, to convective systems with disconnected thermal pillars of smaller scale, which travel in the same direction of the prevailing wind. The classical sheltering effect, which for flows that are steady 'in mean' simply consists of an increased deflection of the leading buoyant jet with respect to the trailing ones, is taken over by a variety of new phenomena, including (but not limited to) fast plume removal-rebirth mechanisms (with local increase in the velocity frequency and shrinkage in the related amplitude), 'bubble' formation-rupture and local departure of the frequency spectrum from the Kolmogorov similarity law.
\end{abstract}

Key words: Plume Dynamics, Cross flow, Jet coalescence, Chaos

\section{Introduction}

Sets of multiple buoyant plumes interacting with a cross flow are widespread in the natural and artificial environment and can be categorized under different perspectives. Common examples in nature are volcanic gas eruptions (Valentine and Wohletz ${ }^{1}$; Kaminski et al., ${ }^{2}$ ) and hydrothermal vent flows in the deep ocean. They are also a typical feature of modern society's fluid waste disposal methods as witnessed by the various visible gaseous emissions into the atmosphere from domestic and industrial smokestacks (McGrattan et al., ${ }^{3}$ ) and from cooling towers or mobile exhausts. Other 'non-visible' examples are represented by the releases of liquid into coastal water, rivers and lakes from a variety of (industrial, municipal and agricultural) sources, mining and oil extraction operations (Jirka ${ }^{4}$; Tomàs et al., ${ }^{5}$ ), chemical reactors and various plants for waste treatment and desalination facilities (Oliver et al., ${ }^{6}$ ). Many other variants can be found in the specific field of 
energy production, where configurations with multiple sources of buoyancy are a characteristic feature of thermal discharges from nuclear and fossil-fueled electricity generation plants (Martineau et al., ${ }^{7}$; Lee and Asce ${ }^{8}$; Fregni et al., ${ }^{9}$ ). They can also exist at a smaller scale in typical problems relating to the cooling of computer mother boards and related CPUs and memories (Sun and Jaluria $^{10}$; Biswas et al., ${ }^{11}$ ) or in combustion chambers as a result of the presence of holes or orifices for fuel injection and dilution (Issac and Jakubowski ${ }^{12}$; Baltasar et al., ${ }^{13}$ ). Similar concepts also apply to the manufacturing industry, where gas furnaces are commonly used for the heat treatment of metals (Viskanta ${ }^{14}$ ). In the built (civil engineering) environment, such phenomena are widespread in emergency ventilation and air conditioning systems in buildings (Venkatasubbaiah and Jaluria ${ }^{15}$; Subudhi et al., ${ }^{16}$; Morsli et al., ${ }^{17,18}$; Harish ${ }^{19}$ ) and can be found, in general, in every technological situation in which a heat exchanger is required.

Given the diversity and rich spectrum of circumstances in which multiple buoyant plumes interacting with a cross flow can be encountered in nature and the myriad technological applications briefly reviewed above, generalizations are rather difficult. Many situations are possible in principle depending on the specific case considered, namely, the distribution of buoyancy sources and the properties of the 'external' flow.

In the present work we expressly concentrate on circumstances for which such sources have essentially a thermal nature (thermal buoyancy). Even with this limitation, however, the subject still displays a plethora of variants. The 'receiving' ambient can be characterized by its own temperature distribution, velocity profile and boundary conditions, which are not 'unique'. The temperature may be constant, display a stable thermal stratification, or even an unstable distribution (leading to the onset of massive thermal convection). In terms of velocity, similarly, the ambient fluid may be in stagnant conditions or support a laminar (uniform or sheared) flow. In terms of physical 'boundaries', the domain may vary from 'unbounded' conditions to a geometry partially or totally delimited by solid walls (i.e. an enclosure or cavity). Moreover, the plume dynamics may be steady in mean or completely unpredictable.

These simple arguments explain why the subject has so far resisted a deep and exhaustive analysis. Over the years it has been approached for fundamentally different reasons by distinct research groups with very different backgrounds. Trying to filter out differences and concentrate on common aspects, (and leaving aside for a while the experimental approach, for which the interested reader is referred to, e.g., Smith and Mungal ${ }^{20}$ and references therein and Keramaris and Pechlivanidis ${ }^{21}$ ), we start from the simple realization that the methods elaborated so far to tackle these problems can easily be classified or split into four main general categories, namely: 1) dimensional considerations and OMA (order of magnitude analysis); 2) potential (analytic) flow theory; 3) integral methods of various types and 4) approaches based on the discretization and solution of the governing balance equations for mass, momentum and energy (including mathematical extensions to account for turbulent behavior). While dimensional considerations and the ensuing OMA, in general, provide useful information on 'characteristic quantities' and related functional dependences (Wright ${ }^{22}$; Fischer et al., ${ }^{23}$ ), the other strategies can be used to address other (more subtle) aspects of 
the considered problem. Among them, the evolution of the trajectories of the plumes (typically a topic of great interest in the context of all these studies) and the interaction of initially distinct plumes (this being required for accurate prediction of mixing phenomena occurring in the flow, which eventually lead to dilution or plume 'concentration').

Purely analytical approaches have also enjoyed a widespread use especially for configurations in which a limited number of plumes or jets are present in the physical domain (Jirka and Fong ${ }^{24}$ ). In this regard, in particular, two developments were worth of attention. Kaye and Linden ${ }^{25}$ considered two plumes and assumed them to be advected towards each other by the induced entrainment velocity computed by a potential flow theory. Later Lai and $\mathrm{Lee}^{26,27}$ have proposed a general semianalytical model for the dynamic interaction of multiple buoyant jets (in stagnant ambient conditions) based on a distribution of point sinks and doublets along the jet trajectory with the interaction being assumed to be governed by the inviscid external flow produced by the jet group. With such strategy, the trajectories were determined via the iterative solution of an integral buoyant jet model and by tracking the changes in the external entrainment flow and dynamic pressure fields (the velocity and concentration fields of the jet group being finally obtained by applying a momentum or kinetic energy superposition principle valid in the limit of potential flow).

For what concerns the integral methods, a large cross section of fundamental research in this field for non-stagnant ambient conditions (i.e. in the presence of a cross flow) can be found in the book by Lee and $\mathrm{Chu}^{28}$, and in Jirka ${ }^{4}$ and Lee and $\mathrm{Asce}^{8}$ (the reader being also referred to Isaac and Schetz $\left.^{29}\right)$. A number of them have been developed in a Lagrangian framework in which the generic plume or jet fluid element is assumed to be transported with a given average local velocity along the trajectory (Frick ${ }^{30}$; Lee and Cheung ${ }^{31}$ ) and to undergo during this process a series of transformative events.

The intimate essence of this strategy can be illustrated as follows. From a practical point of view, it is based on the paradigm of a series of different stages. First, the governing equations of motion and turbulent transport (Reynolds averaged equations) are 'integrated' in a local coordinate system (this leads to a system of conservation equations for different flux quantities). Second, the progressive expansion of the turbulent zone is described through a 'spreading' equation (Wood et al., ${ }^{32}$; Chu and Lee, ${ }^{33}$; Chu et al., ${ }^{34}$ ) or through an entrainment model (ambient fluid from the surrounding environment is drawn into the jet through a mechanism of turbulent nature; Morton et al., ${ }^{35}$ ).

All the valuable models (and related findings) described above are applicable to cases in which the flow is steady in mean, i.e. when the ratio of turbulent fluctuations to time-averaged quantities is relatively small (in these circumstances, the plume trajectories do not depend on time, i.e. the resulting pattern can be taken to be 'steady' in the time-averaged space).

Though the theory for steady-in-mean flows has emerged as a leading candidate to analyze these subjects, other works of relevance to the problem also include those where attempts were made to approach it by averaging with respect to 'space', i.e. replacing the discrete set of buoyancy sources with a continuous production of heat along the wall where the heat sources are located. With this strategy it becomes possible to get meaningful information on the convective hybrid regime 
produced by thermal buoyancy and the forced (horizontal) convection (due to a uniform cross flow) using the so-called boundary layer approximation (Sparrow and Minkowycz ${ }^{36}$; Hauptmann ${ }^{37}$; Hieber $^{38}$; Hussain ${ }^{39}$ ).

Whereas all these theoretical models or existing approaches can be organized in well-studied universality classes, unfortunately, the interpretation of experimental realizations is relatively difficult. This is due to the inherent complexity of these flows, which often hide a still-not-fullyunderstood competition of diverse physical mechanisms that determine macroscopic dynamics.

Moreover, all the models discussed above, relying implicitly on the possibility to turn the initial problem into an alternate one in which all the quantities of interest are replaced by the corresponding time-averaged or space-averaged ones, are not applicable when the system enters a peculiar state of turbulence in which the plumes display very unsteady dynamics.

In the present analysis we target expressly such a regime for which there seems to be a significant lack of results in the literature. Our work also proposes widening the range of methodologies used to examine these problems (by including concepts, methods and tools, which are typical of the analysis of chaotic systems).

\section{Mathematical Model and Numerical Method}

\subsection{The considered system}

We concentrate on "line thermals" in cross flow, i.e. the motion produced by a line (assumed to be infinitely extended along a direction perpendicular the plane of the cross flow) of buoyant fluid. More precisely we consider conditions for which the dynamics are completely dominated by plume unsteady creation/surface-detachment and ensuing interaction and mixing phenomena, for which the resulting pattern is expected to share many features with that typical of purely turbulent Rayleigh-Bénard (RB) convection, including its prevailing two-dimensional nature (Refs [40-45]). Due to its disorganized appearance, this convective regime has so far resisted a deeper analysis, and its behavior has often been placed in the category fully developed thermal turbulence.

The considered configuration is shown in Fig. 1. It consists of a rectangular physical domain limited from below by a solid wall featuring a distribution of $\mathrm{N}$ equally spaced heated elements (finite-size buoyancy sources). These solid bodies are assumed to have square shape (i.e. they may be regarded as bars with square cross-section) and uniform temperature, while the underlying floor is adiabatic. A linear velocity profile is imposed at the left side of the domain to simulate the presence of a wind interacting with the considered distribution of hot elements. The other (right and top) sides are treated dynamically as 'open boundaries'. This means that the thermal plumes generated by the set of heat sources periodically positioned along the horizontal direction can leave the system through the upper or the right boundary depending on the prevailing effect (be it buoyancy or forced convection induced by the horizontal wind, respectively, we will come back to these important concepts later). We denote by $\mathrm{d}$ and $l$ the generic size of each square obstacle and the distance 
between two consecutive elements of the array (accordingly, a related non-dimensional parameter is defined as $\eta=l / d$ ). By indicating with $\mathrm{H}$ vertical extension of the fluid domain, other characteristic geometrical parameters are introduced as $\chi=\mathrm{d} / \mathrm{H}$ (nondimensional size of the generic finite-size heated item) and $\mathrm{L} / \mathrm{H}$ (nondimensional horizontal extension of the computational domain). As shown in Fig. 1, the overall length $\mathrm{L}$ can be expressed as $\left[l_{0}+\mathrm{Nd}+(\mathrm{N}-1) l+l_{0}\right]$ where $l_{0}$ is the minimum allowed distance between one of the vertical (left or right) boundaries and the nearest square heat source of the succession.

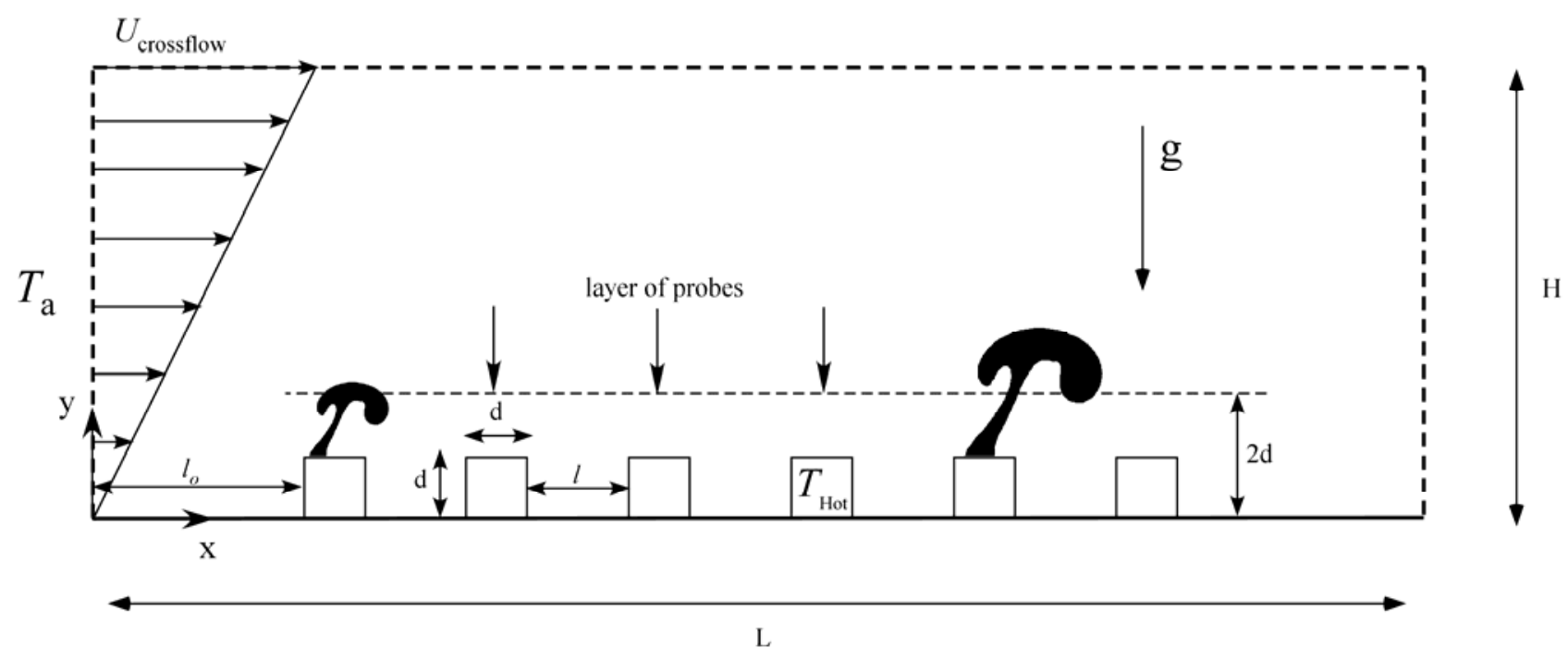

Figure 1: Sketch of the considered problem: an array of evenly distributed heated protuberances with square shape and side $d$ is located at the bottom of a physical domain (solid and dashed lines indicate solid and free boundaries, respectively); the distance $l$ separating a square element from the next one in the downstream direction is constant.

For brevity we confine our attention to a fixed value of the heated body side, namely, $\chi=1 / 12$. Moreover, the number of heat sources $\mathrm{N}$ of the array is set to $\mathrm{N}=10\left(l_{0}=1\right)$. However, important degrees of freedom are introduced in the analysis by changing the spacing parameter $\eta(\eta=1,2$ and 3 ) and allowing the characteristic flow numbers (these being the so-called Reynolds and Rayleigh numbers, for forced and buoyancy convection, respectively), to span relatively wide ranges (the reader being referred to Sect. 2.3 for a proper definition of these characteristic parameters and detailed information about the considered intervals).

As we will show in detail in Sect. 4, the use of surrogate models like that shown in Fig. 1 allows more efficient exploration of the space of parameter and can support a multifaceted spectrum of different research initiatives. As a concluding remark for this section, we wish also to highlight that this configuration complements from a theoretical point of view the equivalent 'internal flow' problem, namely, the rectangular channel with streamwise-periodic ribs mounted on one of the principal walls. This companion subject relating to flows over and around wall-mounted obstacles 
has attracted significant interest over recent years owing to its important technical and industrial applications (related results are useful to improve the designs of the internal surfaces of pipes and a variety of heat exchangers, e.g., gas turbine blade cooling passages and serpentine cooling channels, advanced gas-cooled nuclear reactor fuel elements, solar collectors and cooling systems for electronic devices). There are indeed numerous valuable publications in which 'flow interruption' created in flow passages at periodic intervals (by means of ribs with various possible shapes) was intentionally used to promote turbulence and/or enhance convective heat transfer in ducts and channels (these roughness elements can invariably promote flow reversal in the gaps between them thereby breaking the laminar sub-layer of the internal flow, see, e.g., Refs [46-52]). This is the reason why these elements are also known as "turbulators". Though we will refer afterwards to some of these studies, however, most of them have been limited to isothermal flows or situations in which thermal buoyancy is negligible (temperature considered as a 'passive scalar'), which may be regarded as a distinguishing mark with respect to the specific problem considered in the present work (where the repeated hot items installed on the horizontal wall play the active role of 'plume generators').

\subsection{Large Eddy Simulation: Principles and Model}

When regimes of chaotic hybrid convection are entered, unfortunately, the application of integral methods such as those described in the introduction (implicitly relying on the possibility to consider the plumes as separate entities even though they are bended as a result of the cross flow) is no longer an option. Given the dominant role of plume (and related vortices) coalescence and splitting phenomena with respect to simple localized entrainment effects, this also requires some change or shift in the most relevant turbulence model to be adopted.

Simulation of turbulent fluid flows is generally made very complex by the wide range of scales typically involved in these problems. Approximations of such flows can be achieved numerically by solving directly the unsteady balance equations with an adequate number of grid points, i.e. by direct numerical simulation (DNS). Alternative approaches can yet be obtained by filtering the equations in time and introducing a closure model for the Reynolds stresses (RANS approaches) or filtering the equations in space and introducing an adequate representation for the sub-grid dynamic stresses and heat flux (the so-called LES approaches).

Direct solution (DNS) of the governing partial differential equations (the Navier-Stokes equations complemented by the energy equation for the diffusive and convective transport of heat) is very demanding (often prohibitive) in terms of computational resources and simulation time required ${ }^{53}$. In practice, the time-averaging approach, where correlation terms (i.e. the Reynolds stresses) are modelled, is not always a viable alternative because the physics of the various scales that contribute to the correlation terms is not the same $\left(\right.$ Edison $\left.^{54}\right)$.

While RANS have enjoyed a widespread use in the context of integral methods (Ref [4] and references therein) and even in circumstances for which the equations have been approached in 
terms of CFD (cases for which the pattern can be assumed to be steady in mean, see $\mathrm{Yu}$ and $\mathrm{Li}^{55}$; Ma and $\mathrm{Li}^{56}$; Xiao et al., ${ }^{57}$; Harish and Venkatasubbaiah ${ }^{58,59}$; Tsai et al., ${ }^{60}$; Weihing et al., ${ }^{61}$; Lou et al., ${ }^{62}$ ), for the case of highly unsteady dynamics, LES (Large Eddy Simulation) seems to be a more relevant choice, especially when the phenomena of vortex coalescence and splitting become pervasive throughout the computational domain. The LES approach has been successfully applied to pure thermal convection (Eidson ${ }^{54}$; Wong and Lilly ${ }^{63}$; Kimmel and Domaradzki ${ }^{64}$; Pham et al., ${ }^{65}$; Yan ${ }^{66}$; Devenish et al., ${ }^{67}$; Pant and Bhattachary ${ }^{68}$ ), circumstances involving various kinds of jets in cross flow (Morton et al., ${ }^{69}$; $\mathrm{Li}$ and $\mathrm{Wang}^{70}$; $\mathrm{Li}$ and $\mathrm{Ma}^{71}$ ) and flows (without buoyancy) in ducts with "turbulators" (Ciofalo and Collins ${ }^{72}$; Murataa and Mochizuki ${ }^{73}$; Cui et al., ${ }^{74}$; Lohász et al., ${ }^{75}$; Labbé $\left.^{76}\right)$. Though it is not the definite key to the challenge represented by the need to elaborate a compact and self-consistent model, sufficiently general to capture with a single numerical treatment both the large- and small-scale physics, the large-eddy-simulation technique (LES) can overcome some of the above-mentioned bottlenecks, which explains its progressively increasing use over recent years.

The related physical foundation can be found in the generalized idea that turbulence takes a universal (repetitive) behavior in space (homogeneous isotropic turbulence) under a certain length scale (in the so-called inertial range of space scales). This intuition is due to Kolmogorov ${ }^{77-80}$. In this way, small-scale motion (smaller than the so-called "filter width"), which is known to be independent of the boundary conditions, is implicitly removed from the numerical simulation and modelled as a function of local flow conditions. Since the correlation terms include only a modelled estimate of the average effect of the small scales, the resulting velocity fields (generally referred to as the 'resolved' one) can therefore be regarded as estimates of the true solutions to the NavierStokes equations ${ }^{51}$. The study of thermal turbulence is a classical subject for which the LES philosophy is particularly useful. Relevant information about the possibility to use the Kolmogorov argument for the kinetic energy spectrum in a homogeneous fluid undergoing hybrid (mixed) convection can be found, e.g., in the recent theoretical study by Bhattacharjee ${ }^{81}$. There is some general consensus that for turbulence in non-isothermal fluids (with buoyancy) one should take into account that, in addition to the kinetic energy, another form of 'energy' is present whose density scales with the square of the local temperature fluctuations. By calling this the "thermal energy", by analogy with Kolmogorov arguments $\left(\right.$ Kraichnan $^{82}$ ), one may imagine this energy being injected at large length scales (of the order of the vertical distance over which a temperature difference is maintained) and dissipated at small length scales by the thermal diffusivity. Further pursuing the above-mentioned analogy, one may imagine a flux of this thermal energy (the "thermal flux") through the wavenumber scales or length scales. It was shown by Bolgiano ${ }^{83}$ and independently by Obukhov $^{84}$ that in a stably stratified fluid if the thermal flux dominates the kinetic energy flux, then the kinetic energy spectrum becomes $\mathrm{E}(\mathrm{k}) \propto \mathrm{k}^{-11 / 5}$ (Bolgiano-Obukhov or BO spectrum). For a convectively unstable configuration like that considered in the present study, however, as illustrated by Kumar et al., ${ }^{85}$ and Bhattacharjee ${ }^{81}$, due to the dominance of flux of kinetic energy over that of thermal energy, Kolmogorov arguments for the scaling of the energy spectrum are still applicable 
(i.e. $E(k) \propto k^{-5 / 3}$ ), which may be regarded as the necessary theoretical pre-requisite for the application of the standard LES philosophy to mixed convection.

The required quantitative details, which have been glossed over in this section, are put in a proper mathematical context in the next subsection through the ideas originally developed by Smagorinsky ${ }^{86}$ and Lilly ${ }^{87}$ (see also APPENDIX A).

\section{$\underline{2.3 \text { Nondimensional governing equations }}$}

In this section, the preceding theoretical arguments are cast in a form to yield practical governing equations for the considered problem. Referring velocity and temperature to the scales $U_{\text {crossflow }}$ (namely the velocity of the horizontal wind at a distance $H$ from the floor, as shown in Fig. 1) and $\Delta \mathrm{T}$ (the difference between the temperature of the heated bodies $\mathrm{T}_{\text {hot }}$ and the initial temperature of air $T_{\mathrm{a}}$ ), respectively and scaling all distances on the reference length $\mathrm{H}$ and time on $\mathrm{H} / U_{\text {crossflow }}$, the governing equations in nondimensional form (incompressible form with Boussinesq approximation) can be cast in compact form as:

$\underline{\nabla} \cdot \underline{V}=0$

$\frac{\partial \underline{V}}{\partial}=-\underline{\nabla} p-\underline{\nabla} \cdot[\underline{V} \underline{V}]+\left[\frac{1}{\operatorname{Re}}+\lambda^{2} \sqrt{2 S^{2}-\frac{R a}{\operatorname{Pr}^{2} e^{2}} \frac{\partial T}{\partial y}}\right] \nabla^{2} \underline{V}-\frac{R a}{\operatorname{PrRe}^{2}} T \underline{i}_{g}$

$\frac{\partial T}{\partial t}+\underline{\nabla} \cdot[\underline{V T}]=\left[\frac{1}{\operatorname{Pr} \operatorname{Re}}+\frac{\lambda^{2}}{\operatorname{Pr}_{T}} \sqrt{2 S^{2}-\frac{R a}{\operatorname{Pr}^{2} \operatorname{Re}^{2}} \frac{\partial T}{\partial y}}\right] \nabla^{2} T$

where $\underline{V}, T$ and $\mathrm{p}$ are the nondimensional velocity, temperature and pressure, respectively, $\underline{\mathrm{i}}_{\mathrm{g}}$ is the unit vector along the direction of gravity (the reader is referred to APPENDIX A for additional information concerning the quantities appearing in this set of equations). Moreover, $\mathrm{Re}$ and $\mathrm{Ra}$ are the flow Reynolds and Rayleigh numbers, respectively:

$R a=\operatorname{Pr} G r=\frac{g \beta_{T} \Delta T H^{3}}{v \alpha}$

$\operatorname{Re}=\frac{U_{\text {crossflow }} H}{v}$

where $g$ is the gravity acceleration and $\beta_{\Upsilon}, v$ and $\alpha$ are the thermal expansion coefficient (used to account for the linear relationship between the fluid density and its temperature envisaged by the Boussinesq approximation), the kinematic viscosity and the thermal diffusivity of the fluid, 
respectively. Notably, the Reynolds and Rayleigh numbers can be combined into another typical (meaningful) parameter, i.e. the so-called Richardson number:

$$
R i=\frac{g \beta_{T} \Delta T H}{U_{\text {crossflow }}^{2}}=\frac{R a}{\operatorname{PrRe}^{2}}
$$

This number has largely been used in the past to categorize the dynamics of hybrid thermal-forced convection into different regimes (e.g., the so-called 'near and far field' models, Dunn et al., ${ }^{88}$; Lee and $\mathrm{Chu}^{28}$ ) and does also play a crucial role in determining plume instabilities (Vincent and Yuen ${ }^{89}$; Hier Majumder et al., ${ }^{90}$ ).

In terms of Richardson number, the equations can therefore be reformulated as:

$\underline{\nabla} \cdot \underline{V}=0$

$\frac{\partial \underline{V}}{\partial t}=-\underline{\nabla} p-\underline{\nabla} \cdot[\underline{V} \underline{V}]+\left[\frac{1}{\operatorname{Re}}+\lambda^{2} \sqrt{2 S^{2}-R i \frac{\partial T}{\partial y}}\right] \nabla^{2} \underline{V}-R i T \underline{i}_{g}$

$\frac{\partial T}{\partial}+\underline{\nabla} \cdot[\underline{V} T]=\left[\frac{1}{\operatorname{Pr} \operatorname{Re}}+\frac{\lambda^{2}}{\operatorname{Pr}_{T}} \sqrt{2 S^{2}-R i \frac{\partial T}{\partial y}}\right] \nabla^{2} T$

Here we have considered $\operatorname{Pr}=0.71$ (air) and the following intervals for $\operatorname{Ra}$ and $\operatorname{Re}: 3 \times 10^{3} \leq \operatorname{Re} \leq 3 \times 10^{4}$ for $R a=2.7 \times 10^{8}$ and $3 \times 10^{4} \leq \operatorname{Re} \leq 3 \times 10^{5}$ for $R a=2.7 \times 10^{10}$ which correspond to $0.3 \leq(\operatorname{Pr} R i) \leq 30$. Using the size of the square bars as an alternate reference length for the definition of these numbers, i.e. by introducing $R a_{\text {source }}=R a \chi^{3}$ and $R e_{\text {source }}=R e \chi$, these intervals would read $2.5 \times 10^{2} \leq \operatorname{Re}_{\text {source }} \leq 2.5 \times 10^{3}$ for $R a_{\text {source }}=1.56 \times 10^{5}$ and $2.5 \times 10^{3} \leq \operatorname{Re}_{\text {source }} \leq 2.5 \times 10^{4}$ for $R a_{\text {source }}=1.56 \times 10^{7}$.

The governing equations have been solved in the framework of a projection method and using open boundary conditions for the top and lateral boundaries (the reader being referred to APPENDIX B and $\mathrm{C}$ for additional details).

\subsection{Mesh assessment against the Kolmogorov length scale}

Though some general criteria can be found in the literature to estimate the number of points required by LES simulations (see, e.g., Goergiadis et al., ${ }^{91}$; Choi and Moin ${ }^{92}$ and references therein), the application of such rules or estimates to thermal convection or mixed convection is not as straightforward as one would imagine. In natural or hybrid (forced-buoyancy) convection in which thermal plumes play a significant role, the flow is driven by buoyant forces rather than inertial forces, which leads to the need to reconsider some of the typical grid-spacing restrictions required for purely inertial (high-Re) flows (Farhangnia et al., ${ }^{93}$ ). 
This is the reason for which here we rely directly on theoretical rationale derived from the combination of the fundamental principles on which the LES approach is based (i.e. the universal behavior taken by turbulence and the related similarity hypothesis at the root of the Kolmogorov theory), and some (known) typical properties of thermal convection. There is indeed some consensus in the literature that, in the absence of observational (experimental) information to properly tune or constrain the mesh parameters, a judicious use of the LES approach should be based on the following two 'practical' criteria:

- The LES simulations naturally tends to the results of DNS as the mesh size is reduced (see, e.g., Bastiaans et al., ${ }^{94}$;

- The size of the mesh is located in the inertial range of space scales (the filter width or size of the grid cell must lie inside the inertial range of the turbulent energy spectrum)

While the first requirement does not pose significant difficulties (it reduces to a parametric assessment of the influence of the mesh on the solution as the grid size tends to the characteristic Kolmogorov length scale), the second criterion would require 'a priori' knowledge of the extension and location of the inertial range in the overall interval of space scales relevant to the considered problem.

Some information about one of the two extremes of such a range is readily available as several analyses have been appearing in the literature reporting meaningful (quantitative) information on the Kolmogorov length scale $(\zeta)$ for different circumstances.

For forced (e.g. shear) flows, it is known that:

$\zeta_{\mathrm{Re}} \cong \mathrm{Re}^{-3 / 4}$

This correlation might be used when forced flow is dominant, i.e. for $R i<\mathrm{O}(1)$ (see, e.g., Coceal et al., ${ }^{95}$ for $\left.R a=0\right)$. For dominant thermal convection $(R i>O(1))$ similar relationships are available. As an example, for thermal convection being produced by heated vertical walls (gradient of temperature perpendicular to gravity) a quantitative measure of such a length scale can be found in the works by Paolucci ${ }^{96}$ and Farhangnia et al., ${ }^{93}$ :

$\zeta_{R a \perp}=\pi\left(\frac{16 \operatorname{Pr}}{R a}\right)^{3 / 8}$

Similar information about the other fundamental case in which the prevailing temperature gradient is parallel to gravity (heating from below) has been reported by other authors. As an example, following $\mathrm{Kerr}^{97}$ : 
$\zeta_{R a \|}=1.3 R a^{-0.32}$

As comparison of eqs. (11) and (12) leads to the remarkable realization that the heating-from-theside conditions is less demanding in terms of smallest space scale involved $\left(\zeta_{R a \perp}<\zeta_{R a \|}\right)$, and since for the conditions considered in the present work $\zeta_{R a}<\zeta_{\mathrm{Re}}$, in the following we base our arguments entirely on estimates of $\zeta_{R a}$ and considerations relating to the more restrictive situation in which the temperature gradient is parallel to gravity (heating from below effect). For such condition, relevant data about the inertial wavenumber region located between the energy containing and the dissipative ranges have been presented in the very recent study by De et al., ${ }^{98}$. As discussed in the preceding pages, according to the Kolmogorov's hypothesis, the inertial wavenumber region should be located between the macroscopic energy containing scale $\mathrm{H}$ (where kinetic energy is injected into the system) and the dissipative scale (where energy is dissipated due to viscosity), namely the Kolmogorov length $\zeta$. This is equivalent to stating that in the alternative $\mathrm{k} \zeta$ scale (where $\mathrm{k}$ is the wavenumber) most of the kinetic energy of the flow resides at the lower extreme of the scale $(\mathrm{k} \zeta \cong \zeta)$ with dissipation occurring essentially near its higher extreme (i.e. for $\mathrm{k} \zeta \cong 1$ ) (Lohse and $X i a,{ }^{99}$ ). By defining the fraction of cumulative energy $K$ above a given wavenumber $\mathrm{k}\left(f_{\mathrm{K}}\right)$ and the dissipation below it $\left(f_{\varepsilon}\right)$ as:

$f_{k}=\frac{K_{k-\infty}}{K_{0-\infty}}=\frac{\int_{k}^{\infty} K(k) d k}{\int_{0}^{\infty} K(k) d k} \quad f_{\epsilon}=\frac{\varepsilon_{k-\infty}}{\varepsilon_{0-\infty}}=\frac{\int_{k}^{\infty} \varepsilon(k) d k}{\int_{0}^{\infty} \varepsilon(k) d k}$

respectively, De et al., ${ }^{98}$ could identify the extension of the inertial regime $(\Delta)$ as the size of region along the $\mathrm{k} \zeta$ axis delimited by the two points where the curves $f_{\mathrm{K}}$ and $f_{\varepsilon}$ interest the $f=0.1$ axis (see Figure 13 in their work). The extension (upper boundary) of the inertial regime $\Delta$ was found to scale with $R a$ as:

$\Delta=2.22 R a^{-0.196}$ in the bulk

$\Delta=1.01 R a^{-0.18}$ in proximity to the heated wall

Since in our case the physical domain is not bounded from above, which does not allow us to introduce the Rayleigh number in the classical way using as reference length the vertical (finite) extension of the considered enclosure, in the following we expressly rely on the concept of 'local value' of the Rayleigh number. In particular, since our computations are limited to a domain having a given vertical extension, whereas the dynamics of thermal plumes leaving the domain from the top are no longer 'simulated', we base our estimates on the most restrictive possible conditions 
(conservative assumption), namely on estimates of the Rayleigh number based on such extension (which provides a rationale to our decision to define $\mathrm{Ra}$ using eq. (4)).

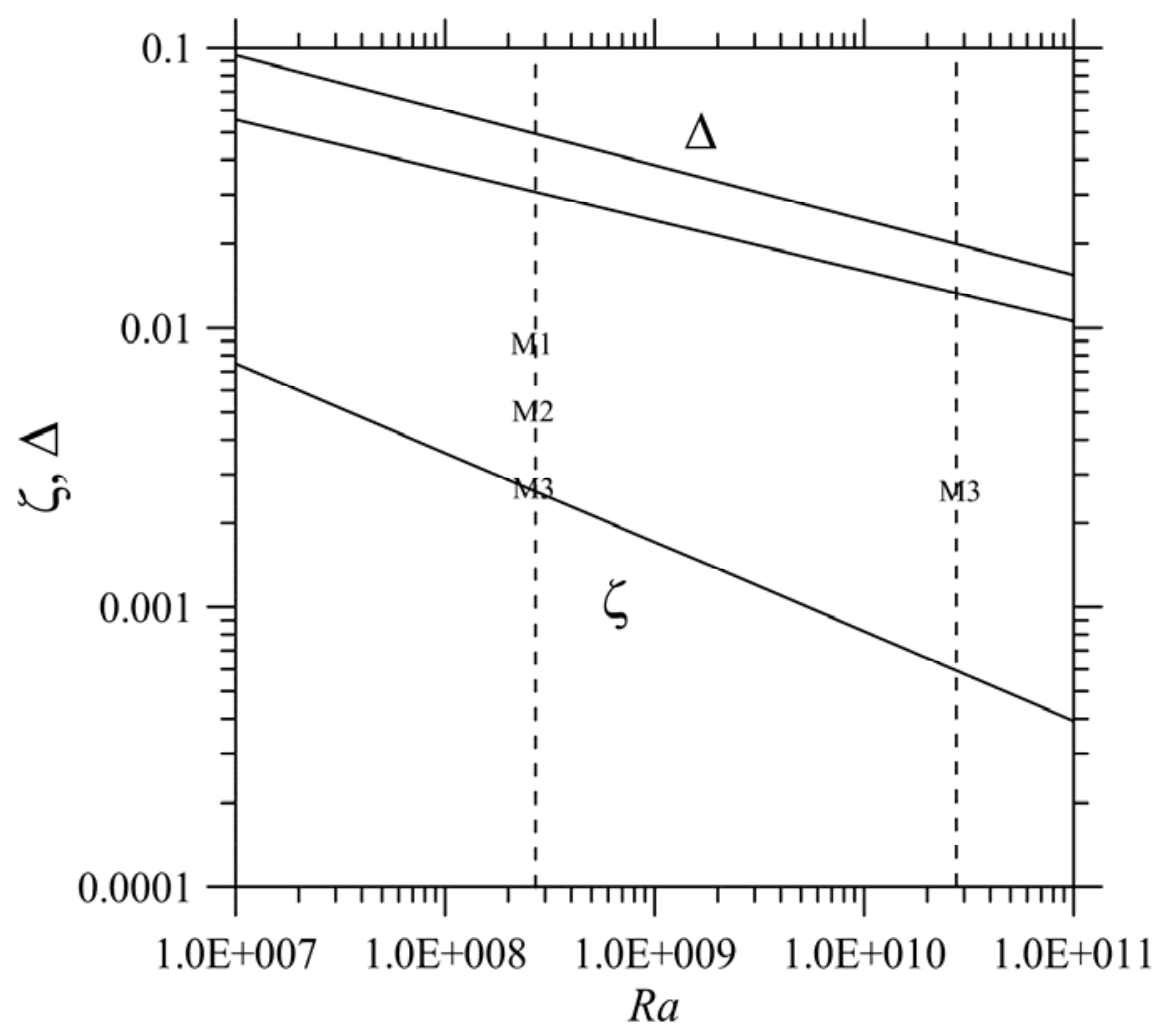

Figure 2: Kolmogorov length scale and extension of the inertial regime $\Delta$ as a function of the Rayleigh number (based on existing correlations).

In such a context, meaningful information is summarized in Fig. 2 where we have reported the size of the uniform mesh used for the present simulations (M1, M2 and M3) together with the available correlations in literature about the Kolmogorov length scale $(\zeta)$ and the amplitude (upper boundary) of the inertial range $(\Delta)$.

Such a figure is useful as it can be used to get 'a priori' some insights into the considered class of problems. Using LES in the region located under the $\zeta$ limit would clearly introduce no benefits in the computations (by contrast, evaluation of the additional terms required by the LES philosophy would increase the overall computational cost); using it in the region overlying the $\Delta$ line would lead to the inclusion into the stress of contributions which have no physical meaning (actually overestimating the extension of the inertial regime). It is the intermediate region that the LES approach enables a number of results to be obtained, which would otherwise be out of our reach. In Figure 2, it can be clearly seen that for $R a=2.7 \times 10^{8}$ the resolution of grids M1 and M2 fall well within the inertial range. A separate discussion, however, is required for the grid M3. While it 
corresponds to the resolution that would be required to capture the smallest scale present in the flow (i.e. the Kolmogorov length scale) for $R a=2.7 \times 10^{8}$, it simply occupies the center of the interval of scales extending from $\zeta$ to $\Delta$ when $R a=2.7 \times 10^{10}$ (a position which was previously taken by the mesh M1 for $R a=2.7 \times 10^{8}$ ). While these arguments make the second of the 'practical' criteria reported before (the size of the mesh has to be located in the inertial range of space scales) satisfied, verification of the first criterion (LES simulations naturally tends to the results of DNS as the mesh size is reduced), requires parametric investigation for different mesh resolution. This is the reason for which we considered 3 different grids for $R a=2.7 \times 10^{8}$. More precisely, we carried out simulations for a representative case $\left(R a=2.7 \times 10^{8}, R e=7.5 \times 10^{3}, \eta=1\right)$ with the grids $M 1$ and $M 2$ in the framework of the LES approach (discussed in APPENDIX A and Sects. 2.3) and on the basis of DNS for the M3 case. We could verify that the solution obtained via DNS with the M3 mesh and that obtained with the LES for M2 were in good agreement (as witnessed by the related frequency spectra reported in Figs. 3). By inspecting this figure (compare Fig. 3a and 3b), the reader will realize that both spectra align with the $\omega^{-5 / 3}$ law predicted by the Kolmogorov similarity hypothesis in an extended range of frequencies (we will come back to this important concept in Sect. 4). The LES simulations performed for M1 (see Fig. 3c), on the other hand, are also important as they demonstrate the ability of LES to effectively capture the dynamics also for scales located halfway the inertial range (comparison between Fig. $3 b$ and $3 c$ provides further evidence for the validity of our entire theoretical architecture).

In the light of these findings and related arguments, all the simulations presented in this work have been performed using the mesh M2 and M3 for $R a=2.7 \times 10^{8}$ and $R a=2.7 \times 10^{10}$, respectively.
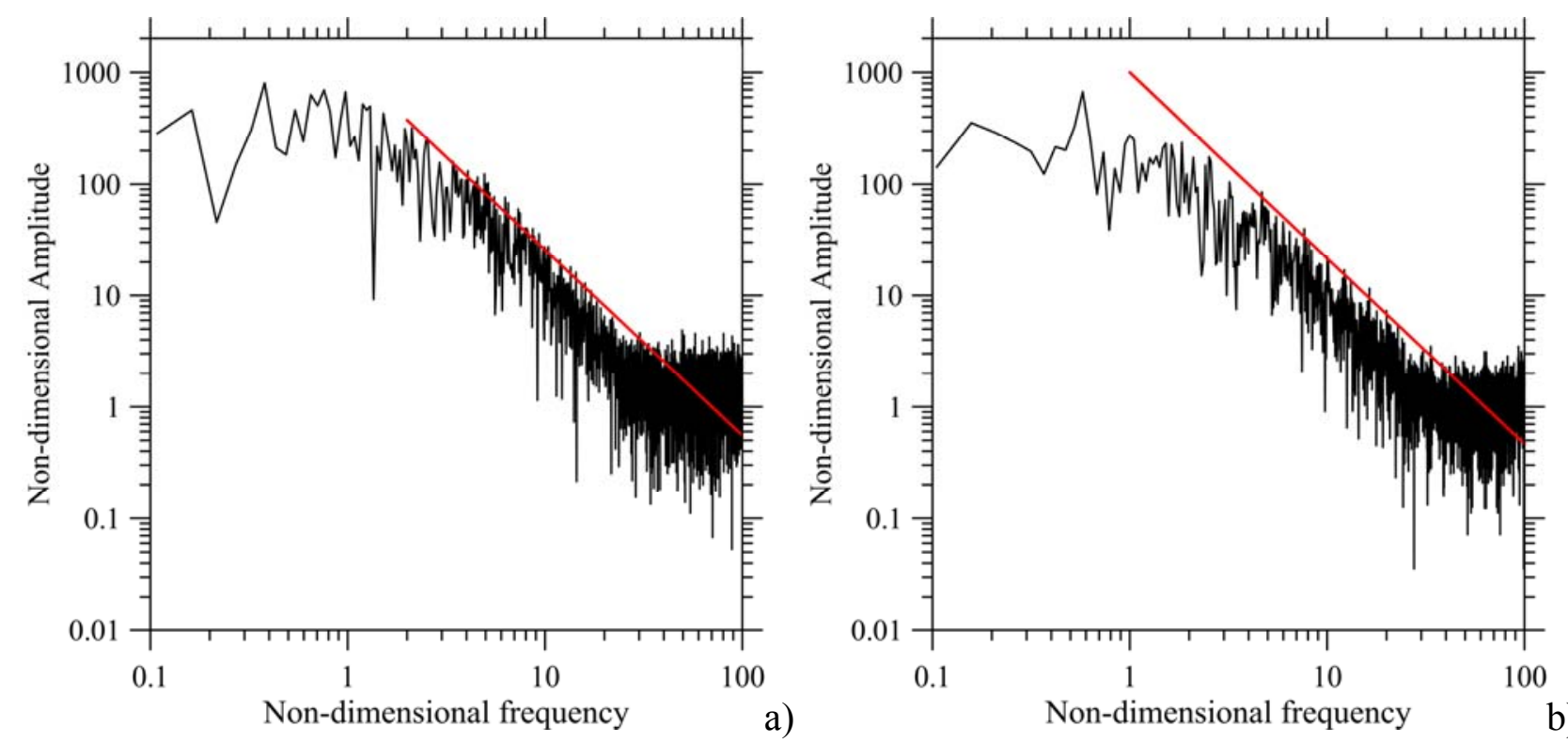

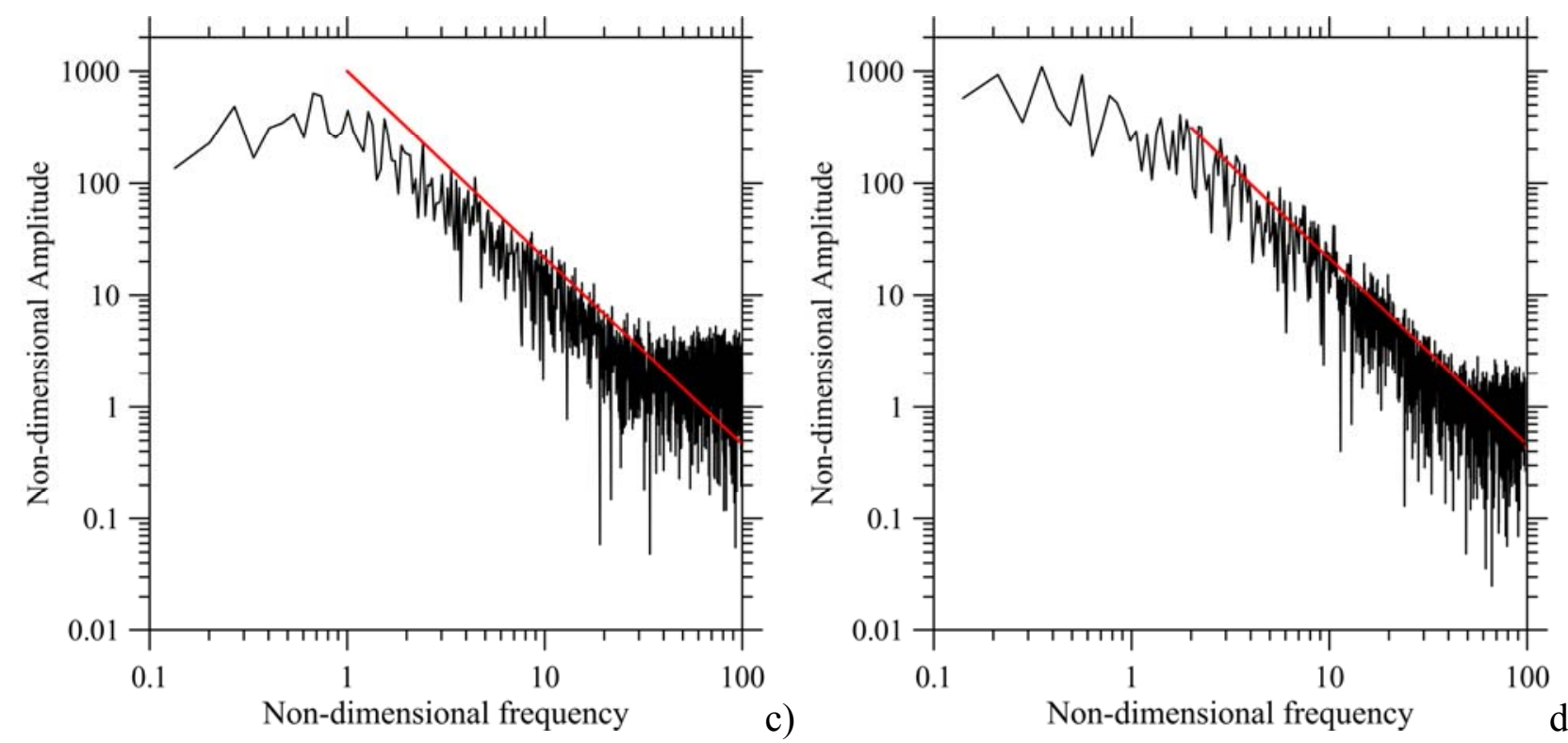

Figure 3: Frequency spectra (probe 5) for $R a_{\text {source }}=1.56 \times 10^{5}, \operatorname{Re}_{\text {source }}=2.5 \times 10^{3}$ and $\eta=1$ : a) DNS with grid M3 $\left(\chi=1 / 12, R a=2.7 \times 10^{8}, R e=7.5 \times 10^{3}\right)$, b) LES with grid M2 $\left(\chi=1 / 12, R a=2.7 \times 10^{8}\right.$, $\left.\mathrm{Re}=7.5 \times 10^{3}\right)$, c) LES with grid M1 $\left(\chi=1 / 12, R a=2.7 \times 10^{8}, \mathrm{Re}=7.5 \times 10^{3}\right)$, d) LES with grid M2 and extension of the domain along the vertical direction increased by $50 \%(\chi=1 / 18)$.

Before starting to deal with the description of the results, we wish to highlight that a final assessment has also been conducted to test the sensitivity of the problem to the vertical extension of the domain (while keeping fixed all the other parameters).

In the light of the arguments elaborated before a scaling relationship accounting for the dependence of the required mesh density on the vertical extension of the domain (keeping fixed its horizontal extension, the size and spacing of the heat sources and the temperature difference between their surface and the fluid) can immediately be provided as follows:

$\Delta y \cong 1.3\left(\frac{g \beta_{T} \Delta T}{v \alpha}\right)^{-0.32} H^{-1}$ for DNS

$1.3\left(\frac{g \beta_{T} \Delta T}{v \alpha}\right)^{-0.32} H^{-1}<\Delta y<2.22\left(\frac{g \beta_{T} \Delta T}{v \alpha}\right)^{-0.196} H^{-0.59}$ for LES

where $\Delta \mathrm{y}$ is the non-dimensional size of the computational cell and $\mathrm{H}$ is the (dimensional) vertical extension of the domain. Put simply this would imply the following: doubling the vertical extension of the domain would require the use of a mesh having non-dimensional $\Delta y$ scaled by a factor $1 / 2$ for DNS (i.e. a number of points doubled) and a $\Delta y$ scaled by a factor in the range between $1 / 2$ and $2 / 3$ for LES. Such a variation, however, should not produce a significant change in the spectrum for fixed size and spacing of sources and fixed value of their relative temperature with respect to the 
fluid. Accordingly, we have used this expectation as a third criterion to judge on the convergence of the overall numerical framework. The outcomes of the simulations conducted by increasing the vertical extension of the domain by $50 \%$ of its initial value while keeping the horizontal length and all the other parameters unchanged (that is by maintaining $R a_{\text {source }}$ and $\mathrm{Re}_{\text {source }}$ constant) are shown in Fig. 3d, where it can be seen (mesh M2) that the variations induced in the spectrum of the signal measured by probes located just above the sources by an increase in $\mathrm{H}$ ( $\chi$ being decreased from $1 / 12$ to $1 / 18$ ) are almost negligible.

With the resolution indicated in Fig. 2, the non-dimensional equations illustrated in Sect. 2.4 were integrated with a non-dimensional time step of the order of $\left(10^{-6}\right)$.

A synthetic description of the salient outcomes of the present study is reported in the remainder of this work (articulated in focused subsections) together with a critical discussion of some accompanying necessary concepts provided to help the reader to interpret the considered dynamics. Results are mainly reported in terms of temperature fields (the related contour legend being shown in Figure 4).

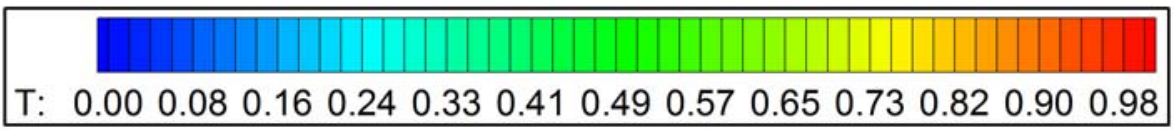

Figure 4: Contour legend (Nondimensional Temperature)

\section{Results}

Though many studies have provided some evidence that for thermal plumes at relatively high values of the Rayleigh number, such as those considered here, three-dimensional structures may appear in the ascending flow (Cortese and Balachandar, ${ }^{100}$; Bastiaans et al., ${ }^{94}$; Plourde et al., ${ }^{101}$ ), as anticipated in Sect. 2.1, we limit to 2D computations. Though this assumption may be regarded as a limitation, in practice, the need for it stems from the two-fold purpose of limiting the (otherwise prohibitive) computational cost required by multi-plume configurations and, at the same time, from our stated intention to elaborate a minimal model allowing us to identify and describe clearly the differences between the dynamics of multiple buoyant jets in cross flows for conditions in which the flow is steady in mean (plume trajectories fixed in time) already considered in past works and the case for which the dynamics of plumes become highly unsteady and unpredictable (present case). The reason of such a choice also resides in the fact that, as anticipated in Sect. 2.1, these systems often display a prevailing two-dimensional nature.

The next step in this hierarchy of models and objectives is represented by the choice of the constants (see APPENDIX A) to be used for the effective application of the LES model.

Still keeping in mind the arguments given above, we rely on values of Cs and $\operatorname{Pr}_{\mathrm{T}}$ which have enjoyed a widespread use in other studies over a wide range of problems and conditions. As an example, though the selection of the proper value to be used for the turbulent Prandtl number in 
order to capture precisely experimental results is a still debated question, in line with the assumptions of Bastiaans et al., ${ }^{94}$ and $\mathrm{Li}$ and $\mathrm{Ma},{ }^{71}$ for the simulation of thermal plumes, and those by Murataa and Mochizuki ${ }^{73}$ for the companion problem relating to turbulent heat transfer in rectangular ducts with transverse rib turbulators we fix $\operatorname{Pr}_{\mathrm{T}}$ to 0.5 . Moreover, following the analyses by Nakayama and co-workers (Nakayama and Nagai ${ }^{102}$; Nakayama et al., ${ }^{103-107}$ ), who performed LESs of turbulent flows in the atmospheric boundary layer with thermal plumes for a variety of conditions $\left(0.12 \leq R i \leq 0.45, \mathrm{Re}=\mathrm{O}\left(10^{5}\right)\right.$ obtaining good agreement with dedicated experiments, in the present paper we assume $\mathrm{Cs}=0.1$ (notably, $\mathrm{Li}$ and $\mathrm{Ma}^{71}$ used the same value of $\mathrm{Cs}$ for pure thermogravitational convection, i.e. $\mathrm{Re}=0$ while Ciofalo and Collins ${ }^{72}$, and Lohász et al., ${ }^{75}$ yet assumed $\mathrm{Cs}=0.1$ for rib-roughened channels in the opposite condition for which there was no thermal buoyancy, i.e. $R a=0$ ).

Prior to expanding on the present results, it is also instructive to briefly provide the reader with some fundamental information about the typical regimes of plume growth as they were categorized by Hier Majumder et al., ${ }^{90}$. For Prandtl numbers ranging from $10^{-2}$ up to $10^{4}$, these authors identified four different regimes of plume growth, which they classified in the Prandtl-Rayleigh number space as $\mathrm{DV}=$ diffusive-viscous regime; IVD=inviscid-diffusive regime; $\mathrm{VND}=$ viscousnondiffusive regime; IVND=inviscid-nondiffusive regime (using the relative importance of viscous and thermal effects as a distinguishing criterion i.e. the strength of gradients of temperature and velocity along the plume). According to these studies, for $\operatorname{Pr} \cong 1$ (the case of gases) the DV regime evolves directly into the IVND inviscid-nondiffusive regime for $R a$ exceeding a value $\cong 10^{6}$. We can therefore expect that the plumes under investigation here $\left(R a>O\left(10^{6}\right)\right)$ will display the typical features of IVND plumes, i.e. thin sharp stems with well-defined caps and lobes that are significantly deformed by vortex structures. The tendency to develop such vortices makes this regime very susceptible to the development of turbulent structures (it is the most turbulent of the four regimes being characterized by fine, turbulent structures that cover a wide range of small scales, Lappa $\left.^{43}\right)$.

\subsection{Plume unsteady dynamics and "pillar" formation}

Following a logical process, with systems of increasing complexity being described as the discussion progresses, we begin our analysis from the less turbulent regime, that is, the flow for $R a=\mathrm{O}\left(10^{8}\right)$. In particular, first we examine a value of the Reynold number for which the dynamics are expected to be relatively similar to those for pure thermogravitational convection, i.e. $\mathrm{Re}=\mathrm{O}\left(10^{3}\right)$ (corresponding to $\mathrm{Ri} \geq \mathrm{O}(10)$ ).

Along these lines, Figs. 5, 6 and 7 show the evolution of the temperature field for $R a=2.7 \times 10^{8}$ and different values of the geometrical parameter $\eta$. As evident in these figures, starting from a situation in which all the fluid has a uniform initial temperature (initial non-dimensional wind temperature $T=0$ as opposed to the temperature of the heat sources set to $\mathrm{T}=1$ ), plumes grow in extension as time increases. They display the typical features of the IVND regime, i.e. a clearly identifiable cap can 
be recognized with shape resembling that of a mushroom (Lappa ${ }^{108}$ ). This specific morphology is due to the interaction of the rising warm fluid with the external environment. Due to viscous friction, the rising fluid tends to be deflected producing a cap that keeps folding with time.

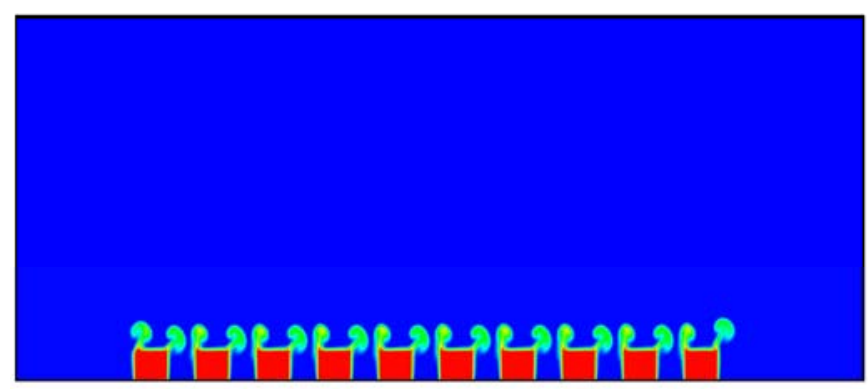

a)

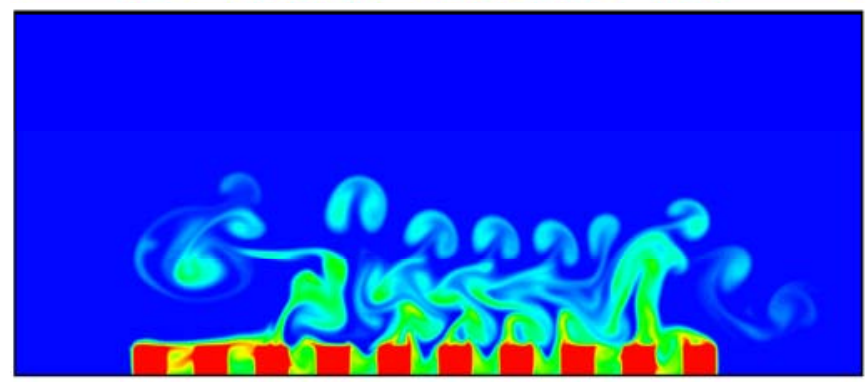

b)

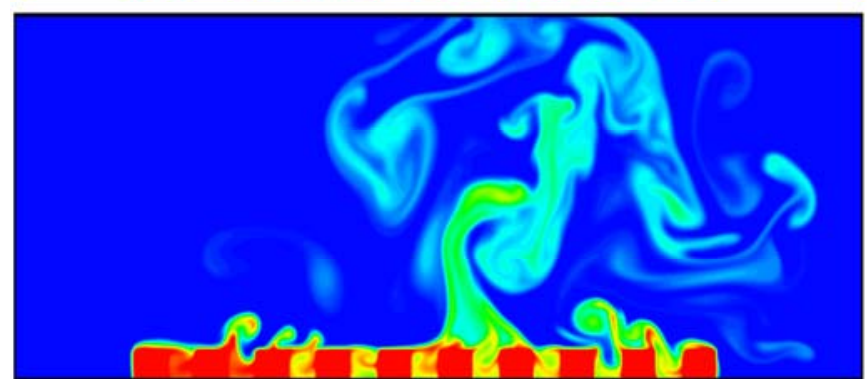

c)

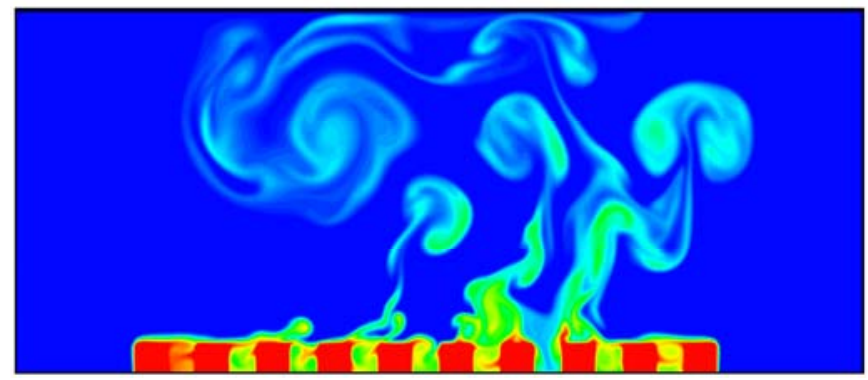

d)

Figure 5: Sequence of snapshots for the case $R a=2.7 \times 10^{8}, \operatorname{Re}=3 \times 10^{3}, \operatorname{Pr} R i=30, \eta=1$ : a) $\mathrm{t} \cong 0.5$, b) $\mathrm{t} \cong 2.5, \mathrm{c}) \mathrm{t} \cong 5, \mathrm{~d}) \mathrm{t} \cong 12.5$.

Remarkably, this means that other than promoting the formation of rising currents of hot fluid, a set of protuberances with square shape periodically arranged in the streamwise direction like those considered in the present work also play the role of vortex (eddy) generators.

For each plume, the cap stays attached to the underlying stalk until at a certain stage, the stalk is broken due to a pinching phenomenon and the plume detaches completely from the solid element (heat source) that produced it. 


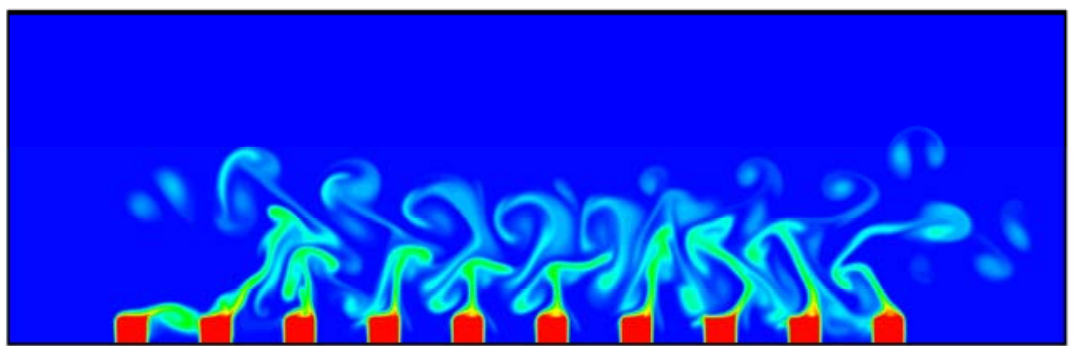

a)

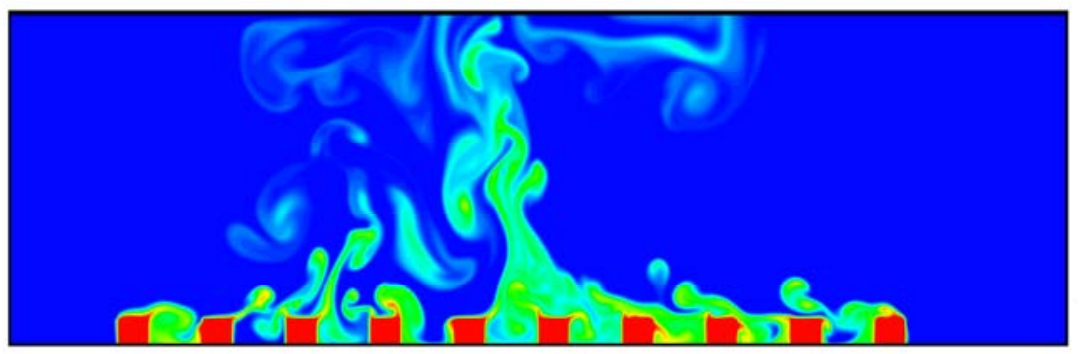

b)

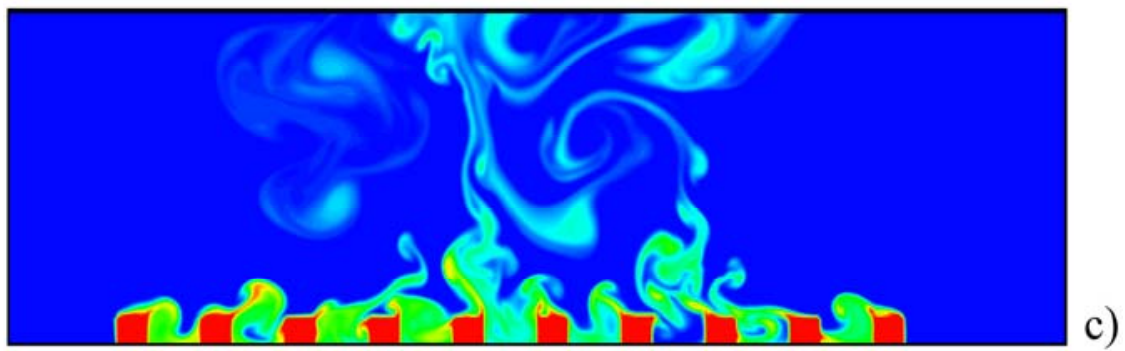

Figure 6: Sequence of snapshots for the case $\left.R a=2.7 \times 10^{8}, \operatorname{Re}=3 \times 10^{3}, \operatorname{Pr} R i=30, \eta=2: a\right) t \cong 2.5, b$ ) $\mathrm{t} \cong 7.5, \mathrm{c}) \mathrm{t} \cong 20$.

Interestingly, given these behaviors, the present plumes might be considered similar to those of solutal nature occurring in other circumstances (see, e.g., Refs [109-112]). When the plume originates from a source having non-infinitesimal vertical size, also the 'shape' of the source (be it a crystal, a droplet or a bar with square cross-section at constant temperature as in the present case) has an effect in determining the dynamics of the plume, especially in the initial stages of evolution. As evident, e.g., in Fig. 4a, the heat released by the square solid elements along their lateral sides causes two small plumes to grow quickly and rise along such sides resulting in two small plumes apparently originating from the upper corners of the element. After some time, however, this memory effect is lost and the plume takes the classical configuration with a single cap that, as explained before, rises and folds in time.

All these descriptions are quantitatively substantiated by the data reported in Figs. 5-7. As time progresses and horizontal shear develops, the plume stems tend to become quite sinuous with vortices being shed from either side of the plume lobes. This behavior is essentially due to a classical shear driven instability (Pera and Gebhart ${ }^{113}$; Hier Majumder et al., ${ }^{90}$; Lappa ${ }^{108}$; Qiao et al., ${ }^{114}$ ). For the so-called natural swaying motion of confined plumes, the reader may also consult Desrayaud and Lauriat ${ }^{115}$. In some cases, as a result of these instabilities, the plume stem becomes so distorted that the local Rayleigh number is supercritical. This leads the original plume to collapse with new plumes originating from the original plume stem (see, e.g., Cortese and Balachandar ${ }^{100}$; Vincent and Yuen ${ }^{116}$; and Shao et al., ${ }^{117}$ ). 


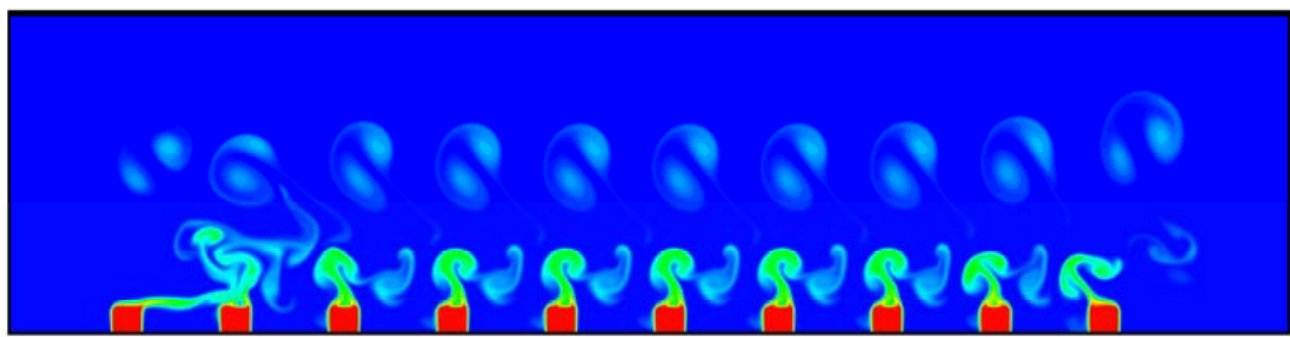

a)
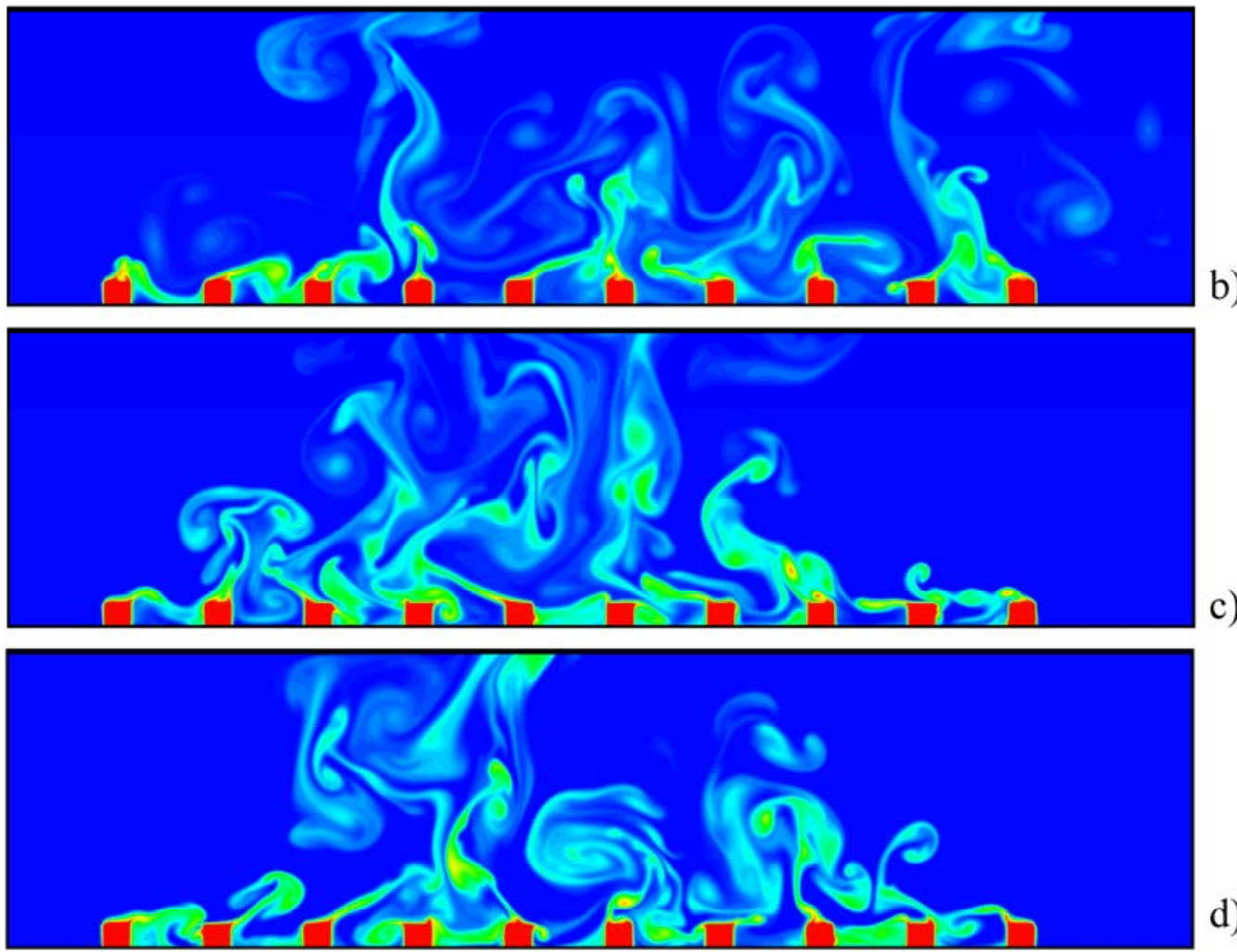

Figure 7: Sequence of snapshots for the case $\left.R a=2.7 \times 10^{8}, \operatorname{Re}=3 \times 10^{3}, \operatorname{Pr} R i=30, \eta=3: a\right) t \cong 2.5, b$ ) $\mathrm{t} \cong 5.0, \mathrm{c}) \mathrm{t} \cong 7.5, \mathrm{~d}) \mathrm{t} \cong 20$.

Moreover, as proven by Hier Majumder et al., ${ }^{90}$, the vortices in the lobes of the IVND plume can form Kelvin-Helmholtz waves, the susceptibility to form such waves being measured by the (local) gradient Richardson number:

$$
R i_{l o c}=\frac{-R i}{\left(\frac{\partial u}{\partial y}\right)^{2}} \frac{\partial T}{\partial y}
$$

where $\partial \mathrm{u} / \partial \mathrm{y}$ is the gradient of horizontal velocity. The instability typically occurs when $R i_{\text {loc }}$ drops below 1/4. Notably, as shown in Fig. 8, the value of the gradient Richardson number can be locally smaller than $1 / 4$ even if $R i>>1$ ( $R i=30 / \operatorname{Pr}$ for these cases). 


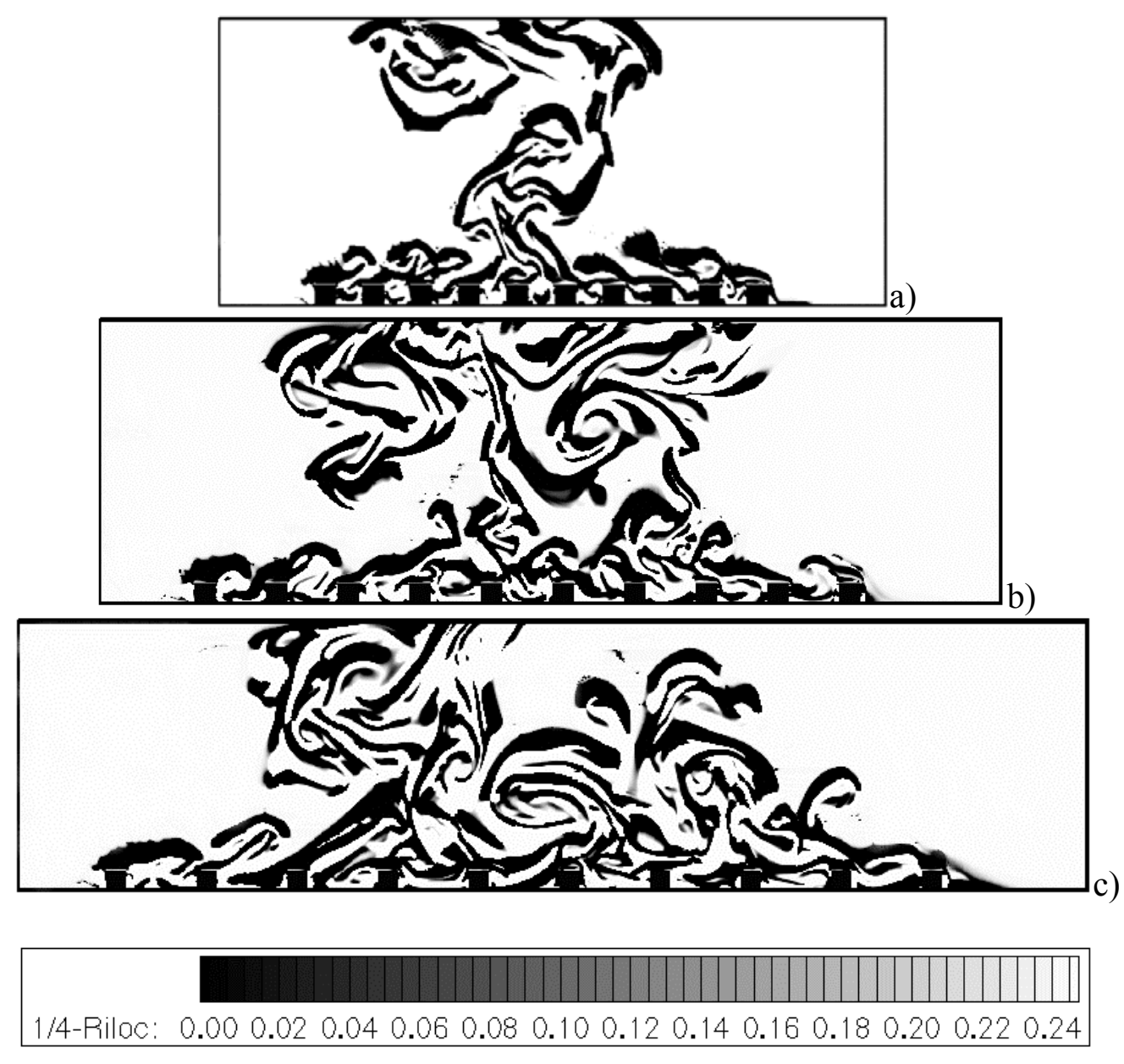

Figure 8: Snapshot of $\Delta R i_{1 \mathrm{loc}}=1 / 4-R i_{\mathrm{loc}}\left(R a=2.7 \times 10^{8}, \operatorname{Re}=3 \times 10^{3}, \operatorname{Pr} R i=30, \mathrm{t} \cong 20\right)$ : a) $\left.\eta=1, \mathrm{~b}\right) \eta=2$, c) $\eta=3$.

In the present study, rather than examining the dynamics of single plumes, we concentrate on the ensemble scenario. Along these lines, Figs. 5-7 are also instrumental in showing that, as a result of the unsteady (but progressive and continuous) formation and detachment of plumes from the heat sources and the tendency of the vortices they carry on to merge, a large scale vertically extended structure is formed approximately at the center of the array of heated bodies (hereafter we will refer to this large-scale convective structure as the central 'pillar').

\section{2. “pillar” migration in cross flow}

The present section continues the investigation into these dynamics by probing the role of the Reynolds number. As Re is increased (the reader being referred to next group of figures, i.e., Figs. 9 , 8 and 9 for $\operatorname{Re}=7.5 \times 10^{3}$ ) the horizontal flow driven by the imposed wind starts to interfere significantly with the spontaneous plume clustering mechanism described in Sect. 4.1.

Notably, for $\operatorname{Re}=7.5 \times 10^{3}$ ( $R i \cong 5 / \mathrm{Pr}$ ), recalling fluid from the right side is no longer possible because of the horizontal rightward flow. This obviously tends to limit the size of the convective structures resulting from the plume spontaneous coalescence process. 
Following up on the previous point, it is also worth pointing out that the emerging structures do not occupy a fixed position. Rather they travel from the left to the right side. Though during this migration process they can still grow by engulfing smaller-scale plumes met along their way, at a certain stage they leave the domain by passing through the right boundary. This process is vaguely periodic as witnessed by the more or less constant number of pillars spreading horizontally in the downstream direction (while a pillar leaves the domain a new pillar is being formed at an upstream location).

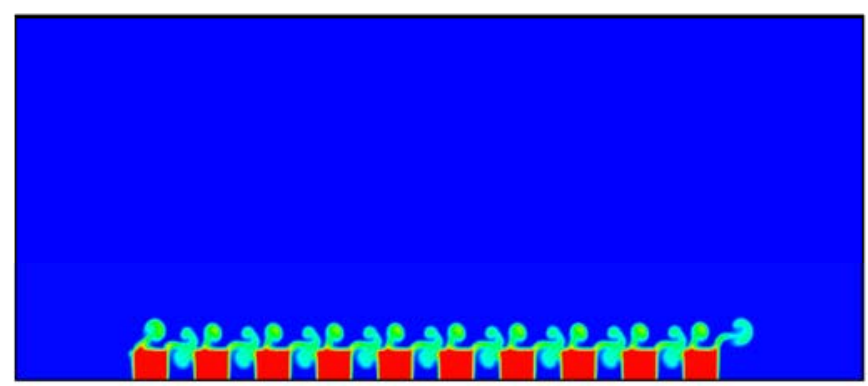

a)

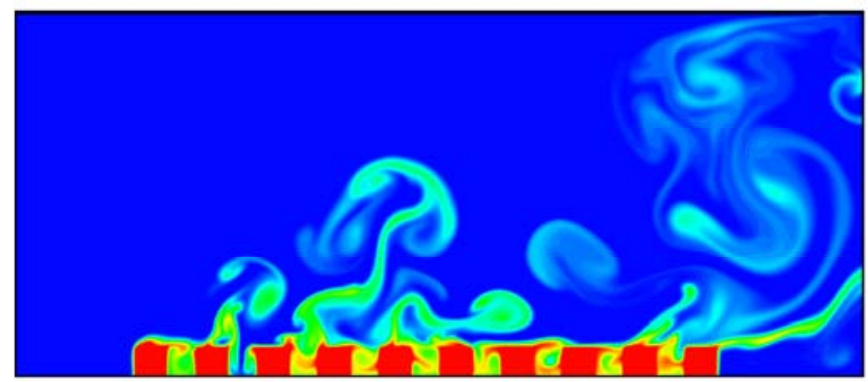

b)

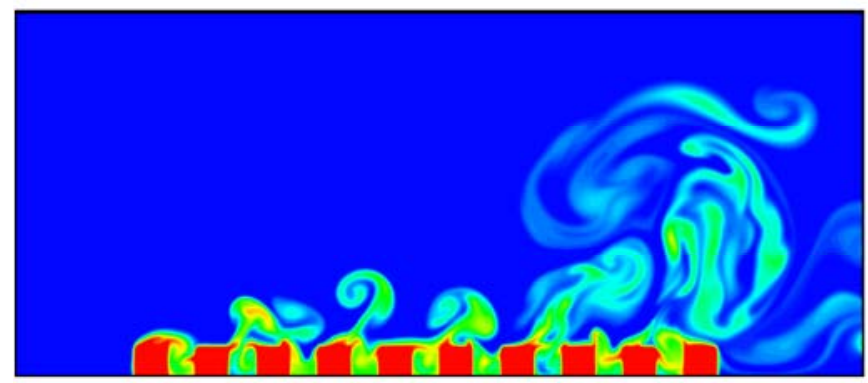

c)

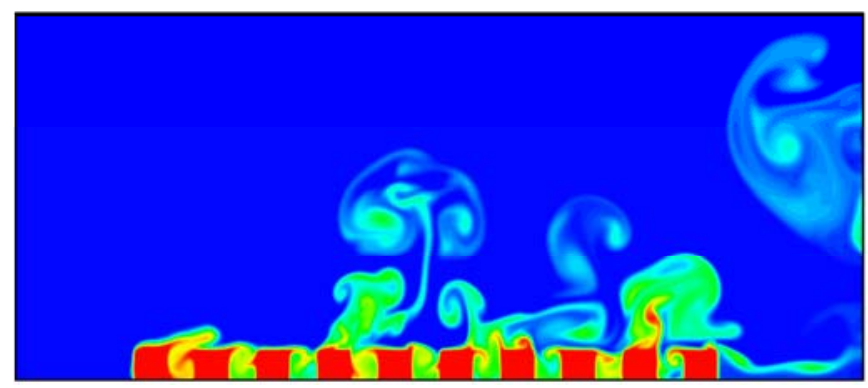

d)

Figure 9: Sequence of snapshots for the case $\left.R a=2.7 \times 10^{8}, \operatorname{Re}=7.5 \times 10^{3}, \operatorname{Pr} R i \cong 5, \eta=1: a\right) t \cong 1.5, b$ ) $\mathrm{t} \cong 15, \mathrm{c}) \mathrm{t} \cong 33.75$, d) $\mathrm{t} \cong 37.5$. 
Interestingly, the number of coexisting pillars (present at the same time above the considered succession of repeated heat sources) changes as a function of the spacing parameter $\eta$. By loosely defining a pillar as a convective structure resulting from the clustering and/or coalescence of plumes and having height at least $50 \%$ of the considered domain vertical extension, on average, a single pillar can be seen in Fig. 9, two pillars in Fig. 10 and 3 pillars in Fig. 11.
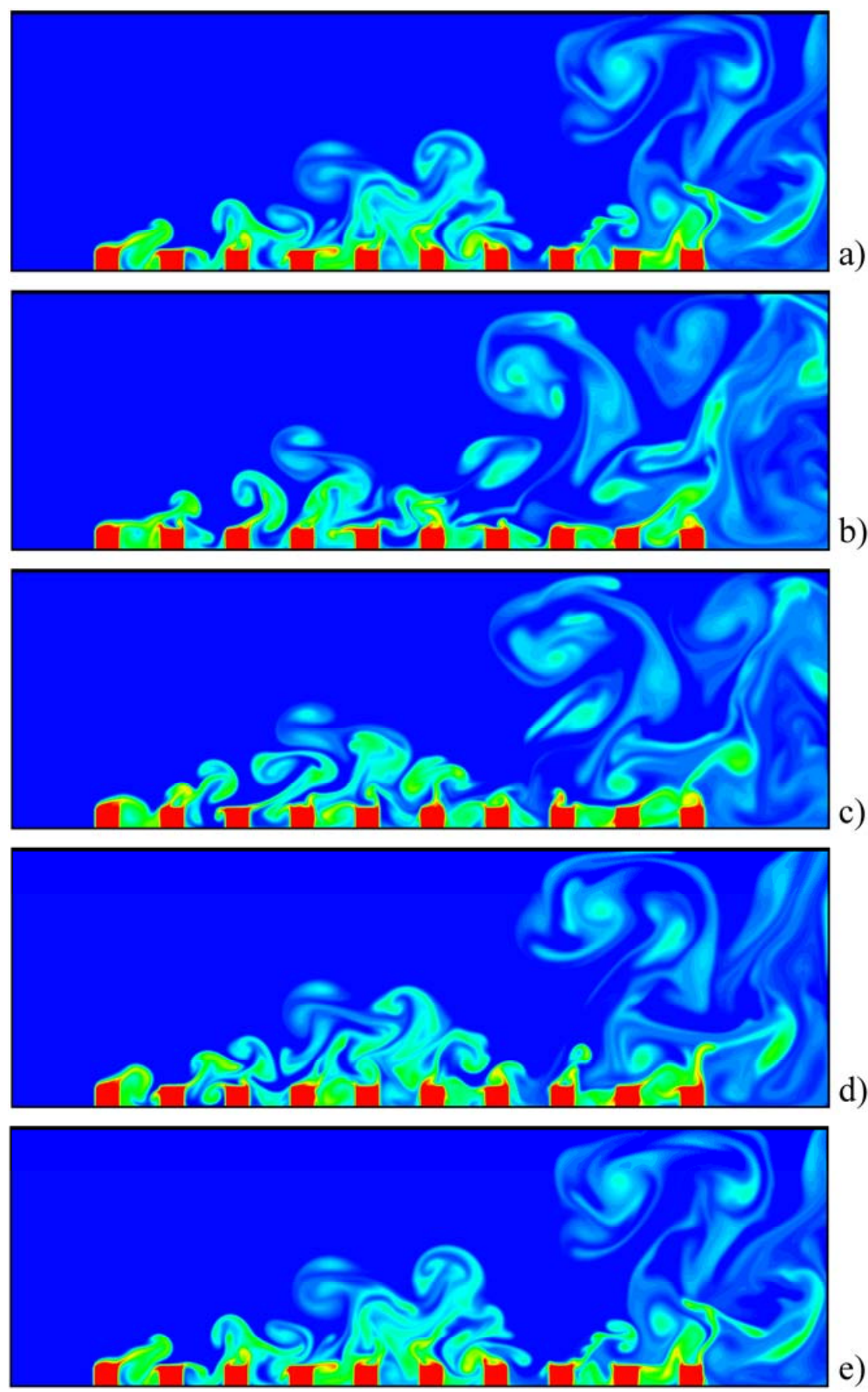

Figure 10: Sequence of snapshots for the case $R a=2.7 \times 10^{8}, \operatorname{Re}=7.5 \times 10^{3}, \operatorname{Pr} R i \cong 5, \eta=2$, initial time $=$ 106.2, $\Delta$ time $=0.75$. 


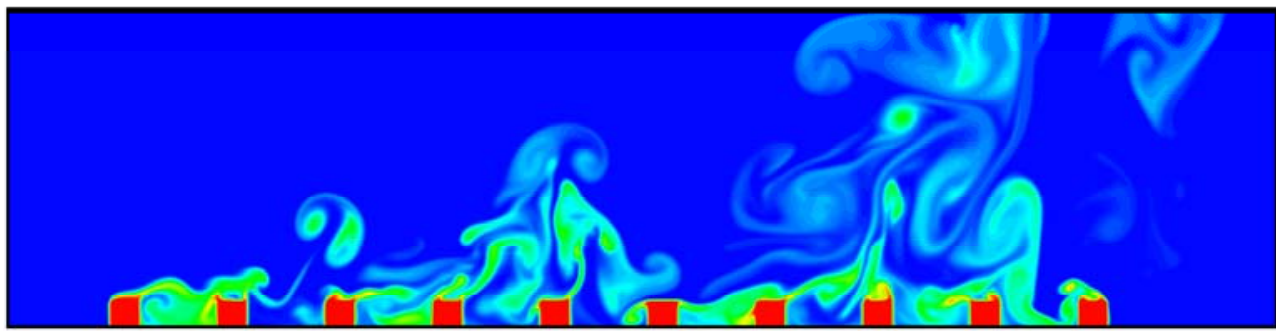

a)

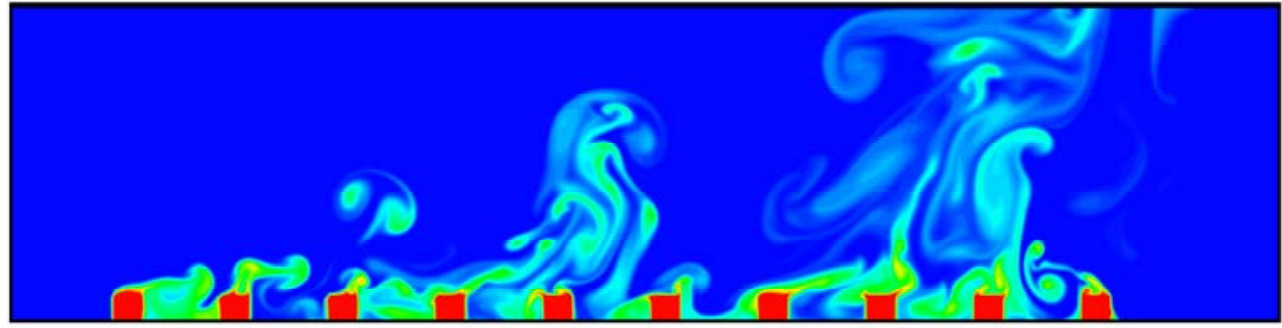

b)

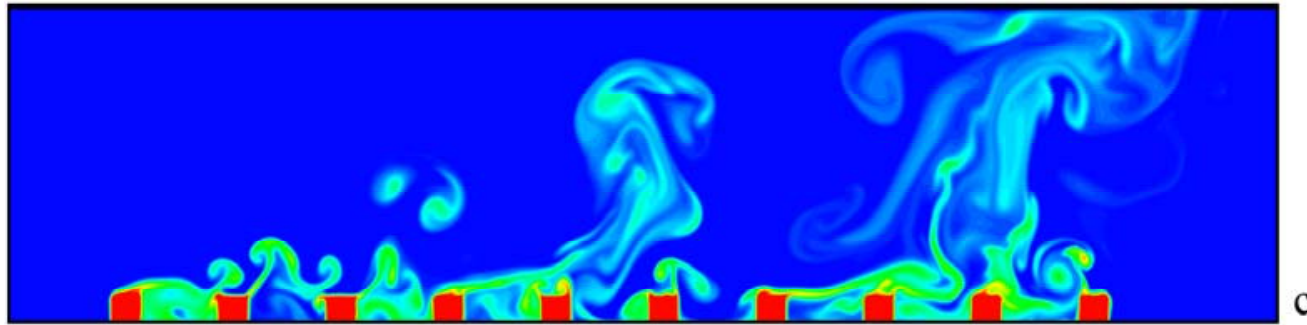

c)

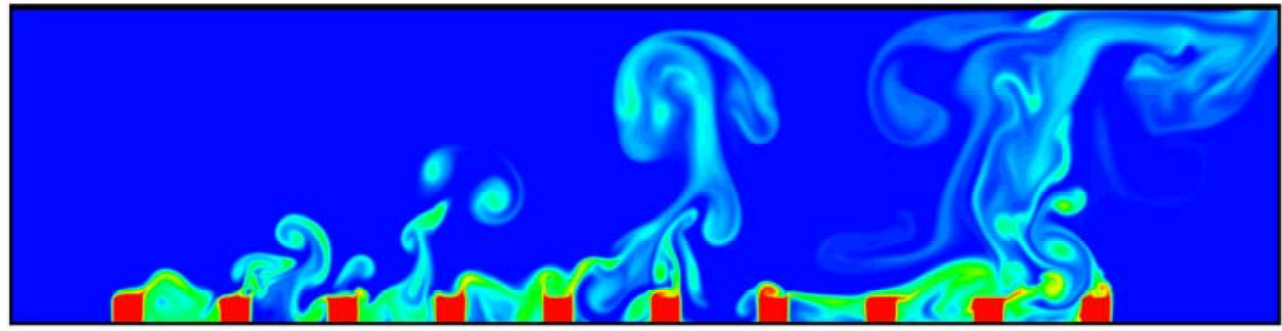

d)

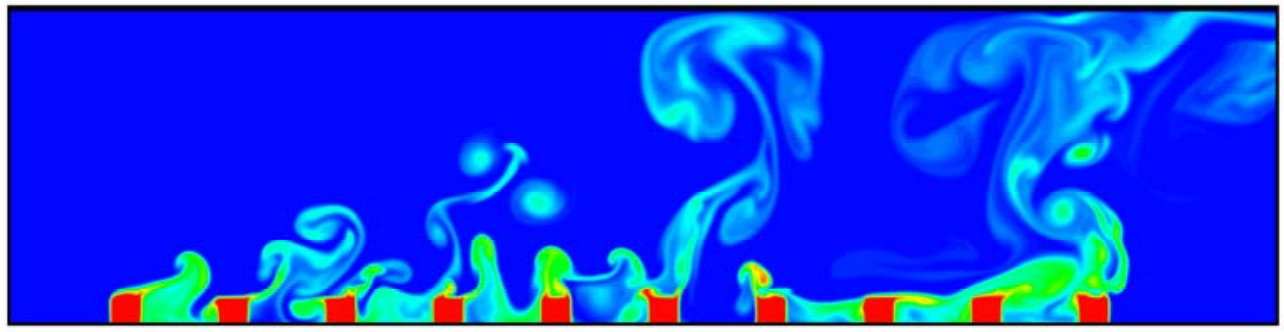

e)

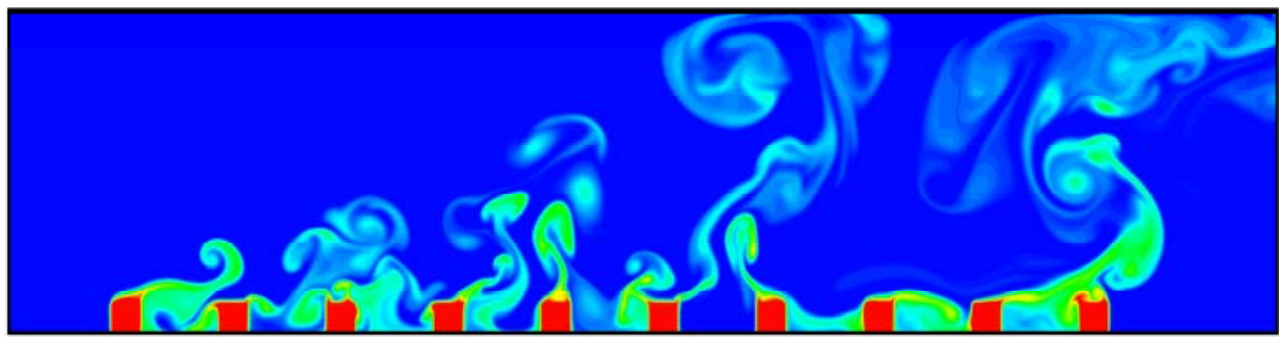

Figure 11: Sequence of snapshots for the case $R a=2.7 \times 10^{8}, \operatorname{Re}=7.5 \times 10^{3}, \operatorname{Pr} R i \cong 5, \eta=3$, initial time $=$ 92.4, $\Delta$ time $=0.75$.

A further increase in $\operatorname{Re}\left(\operatorname{Re}=3 \times 10^{4}, R i=0.3 / \operatorname{Pr}\right)$, however, has a dramatic effect. As witnessed by Fig. 12, in such conditions plumes do not detach and travel to regions where they could eventually interact and coalesce. As soon as they are formed, their shape is asymmetrically distorted by the 
imposed vertical shear. As a result they assume an horizontal elongated configuration which for small spacing of the distribution of the square elements can cause the head of a plume to coalesce with the stem of the plume originating from the next (in the downstream direction) heat source. Though some localized thermal 'peaks' can still be seen along $\mathrm{x}$ as a result of the tendency of warm fluid to rise, horizontally extended regions with more or less uniform temperature are formed.
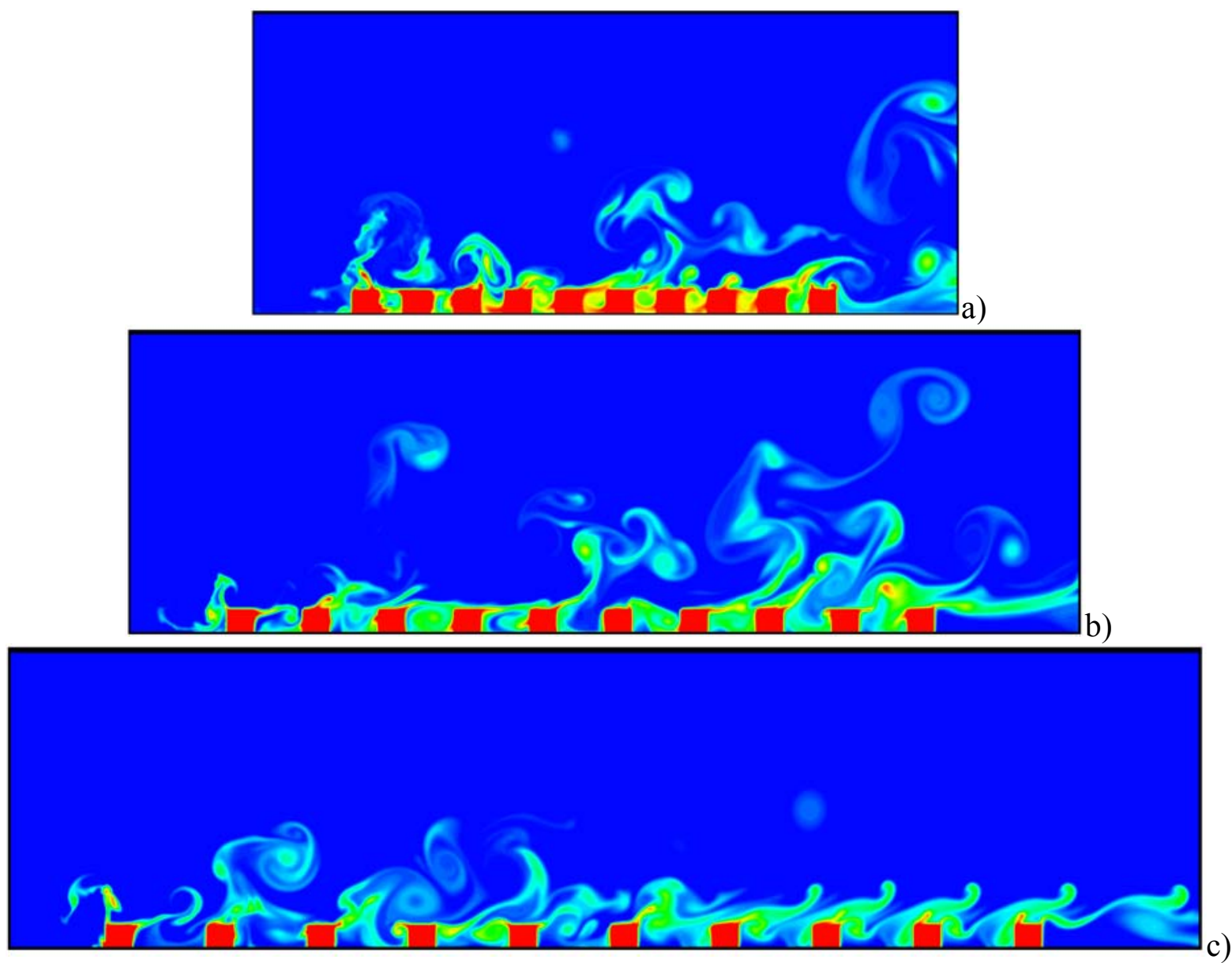

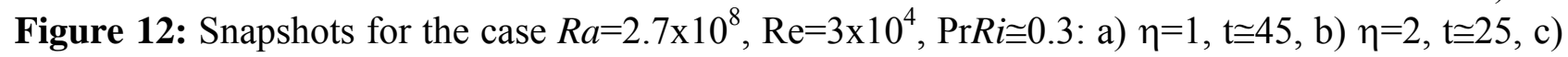
$\eta=3, \mathrm{t} \cong 22.5$.

In practice, for such a small value of the product PrRi, the process is so fast that plumes have no time to travel along the vertical extension of the domain (where they could undergo shear driven instabilities and split). All the heat released in the domain by the heat sources essentially leaves it through the right outflow boundary (Fig. 12). This situation is formally akin to that occurring in the limit condition in which no thermal buoyancy is present, e.g., forced flow in ducts with sparsely distributed ribs used to modulate the fluid current in proximity to the wall. As shown by several investigators (Cui et al., ${ }^{74}$; Fouladi et al., ${ }^{51}$ ), in those conditions the flow continuously separates and reattaches between adjoining protuberances. In the present case, however, in addition to the blockage locally produced by the items mounted in a direction perpendicular to the main flow, there is a significant contribution brought to the separation process by thermal plumes, i.e. by buoyancy effects. 


\subsection{Asynchronous oscillations and emerging anisotropy}

The foregoing discussion has deliberately been limited to illustrating the macroscopic patterning behavior. However, a fruitful alternative for obtaining insights into the considered phenomena is to analyze the velocity signals measured by probes located above each of the items of square crosssection regularly arranged along the bottom wall (at a distance $\mathrm{d}$ from the top wall of each of them, as shown in Fig. 1). As an example, Fig. 13 refers to $\mathrm{Re}=7.5 \times 10^{3}$, i.e. $\operatorname{Pr} R i \cong 5$.
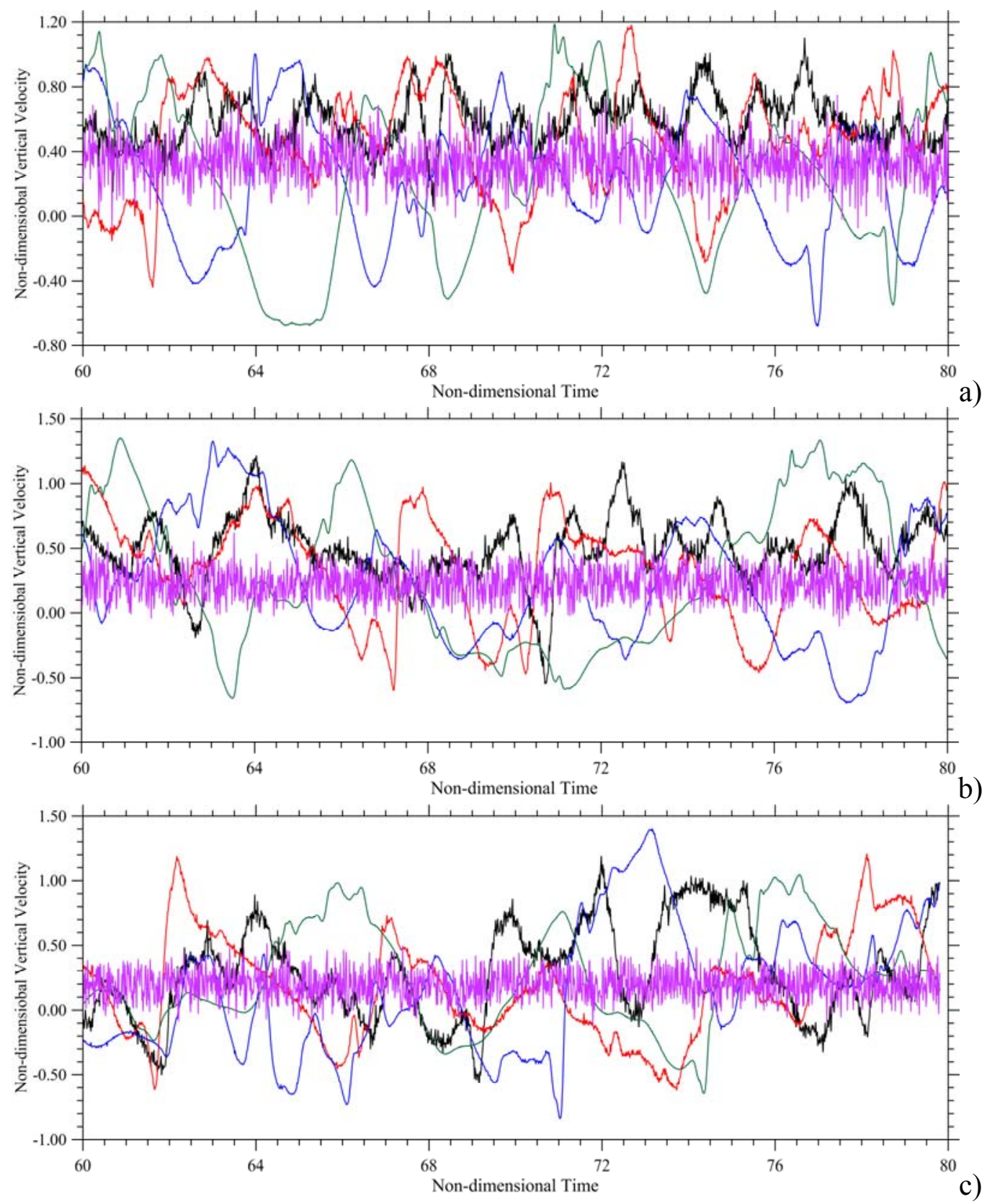

Figure 13: Signals provided by probes located above each heated item (purple, black, red, blue, green lines corresponding to probes $1,3,5,7,9$, respectively) for $R a=2.7 \times 10^{8}, R e=7.5 \times 10^{3}(R i \cong 5 / P r)$ : a) $\eta=1$, b) $\eta=2$, c) $\eta=3$. 
These figures are extremely interesting for the insights they provide into the asynchronous nature of the plume generation and detachment dynamics (the emission of plumes at different stations is not synchronous in time). Moreover, though the distribution of signals is relatively involved (reflecting the turbulent nature of the flow) some important general characteristics can be identified. The reader will indeed realize that, regardless of the considered value of the spacing factor $\eta$, the complexity of the signals tends to be mitigated as the distance from the inflow section increases (in other words, if the signal relating to a station (i) is considered, the signal relating to the next probe $(\mathrm{i}+1)$ in the downstream direction is relatively simpler). This is very evident in Fig. 13; as an example, the high frequency components clearly visible for probes 3 and 5 (black and red lines) are no longer present when probes 7 and 9 are considered (blue and green lines are much 'smoother'). This information can also directly be gathered from Fig. 14a where we have plotted the 'Kurtosis' (or flatness) extracted from the vertical velocity signals reported in Fig. 13 as a function of the probe number. A continuous decrease in the value of this statistical measure of the signal can be seen over the distance that separates probe 2 from probe 8 .

An explanation for this trend can be elaborated in its simplest form on the basis of the argument that the high-frequency fluctuations in the signals relating to probes located closer to the inflow section $(x=0)$ must essentially be ascribed to the interplay which takes place there between the imposed horizontal flow (wind) and the tendency of hot fluid to rise due to buoyancy. Such mutual interference causes continuous production of plumes at a relatively fast rate. As soon as a plume is spread along the horizontal direction by the imposed wind, a new plume is formed at that station. As a result, a high-frequency component enters the spectrum. This component can no longer be seen in the frequency spectrum of probes located further in the downstream direction as in that region the development of plumes is somehow shielded against the incoming horizontal flow by the vortices and resulting involved convective structures located in the upstream direction. Such vortices (induced by the interaction of buoyancy and horizontal wind) essentially cause an upward deflection of the mean wind. This gives plumes located downstream more time to grow before they detach from the heated bodies, which explains why the velocity vertical component displays a less turbulent behavior.

A separate discussion is needed for the signal provided by the first numerical probe. As evident for all cases summarized in Fig. 13, for an intermediate value of $R i(\operatorname{Pr} R i \cong 5)$ this signal presents two clear distinguishing marks. Its frequency is extremely high and, at the same time, its amplitude is relatively limited. In line with the arguments above, the limited amplitude of the signal should be seen as a complete suppression of the possibility for the first finite-size heat source of the succession to develop extended plumes. This behavior yet reflects the extremely counteracting nature established there between the tendency of the heat source to create vertical plumes and that of horizontal wind to remove them (causing their detachment and transport in the downstream direction). Using a more heuristic perspective, it might be argued that since vertical plumes are characterized by strong horizontal shear while horizontal wind favors vertical shear, the two 
mechanisms are incompatible in the sense that their respective transport mechanism exclude each other.
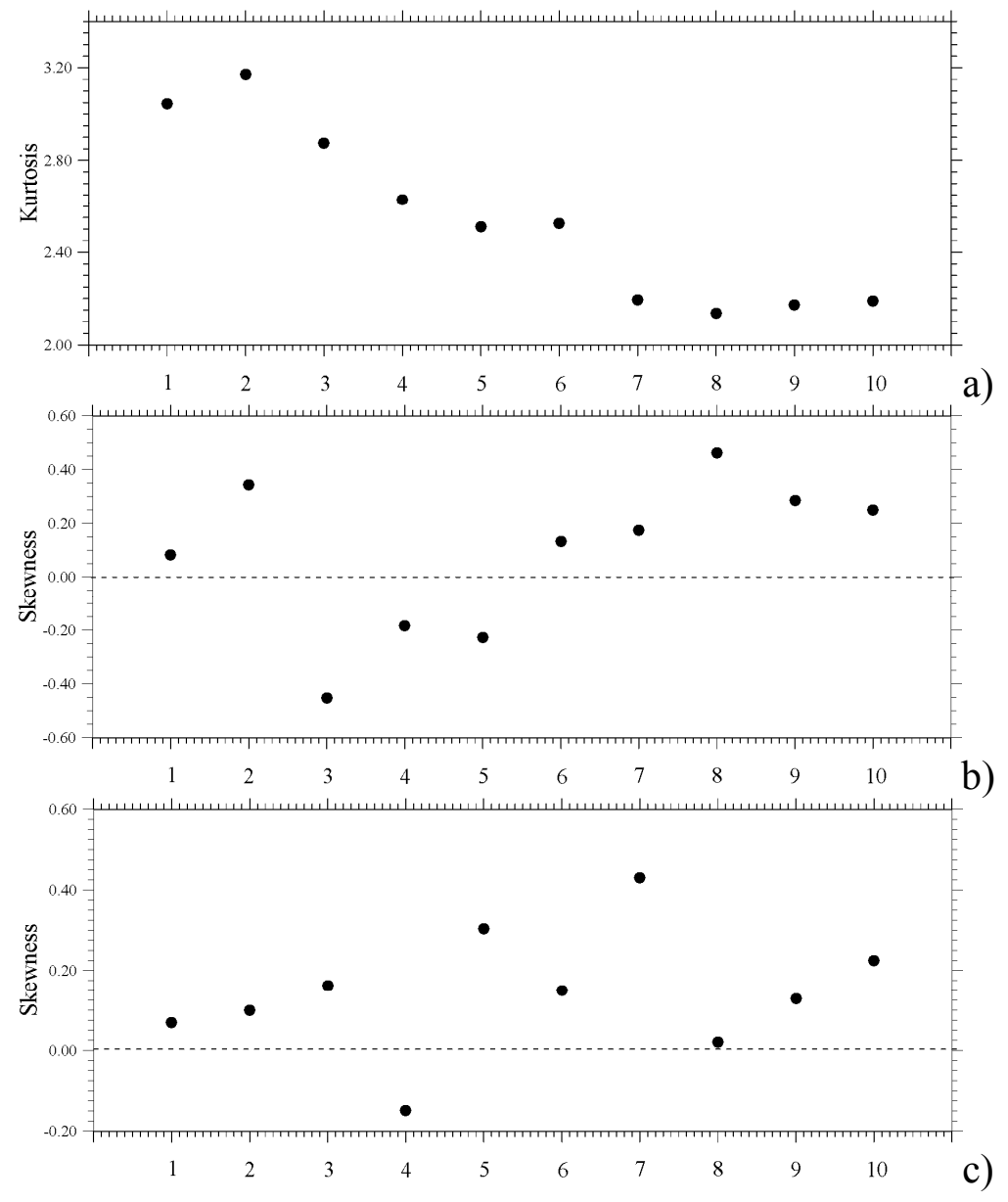

Figure 14: Statistical characterization of plume dynamics $R a=2.7 \times 10^{8}, \operatorname{Re}=7.5 \times 10^{3}$ : a) Kurtosis for $\eta=1, b)$ Skewness for $\eta=2$, c) Skewness for $\eta=3$.

Additional useful insights can be gathered from Fig. 14b and 14c, where, as statistical measure of the highly unsteady state, we have reported (see, e.g., also Zhou and $\mathrm{Xia}^{118}$ and Djenidi et al., ${ }^{119}$ ) the skewness of the signals at different locations for two different values of the heat source spacing (namely, $\eta=2$ and $\eta=3$, respectively). Two trends can clearly be recognized in these plots: 1) the skewness generally increases along the horizontal direction (on average, from probe 3 to probe 8 in Fig. $14 \mathrm{~b}$ and from probe 1 to probe 7 in Figs. 14c), and, 2) negative values tend to disappear on increasing $\eta$.

The former trend can obviously be directly ascribed to the plume coalescence mechanisms, which tend to increase plume vertical extension and strength as the distance from the first heat source become larger. Along the same lines, the latter may be regarded the natural consequence of the reduced ability of plumes and vortices generated by a source to influence (causing a reversal in the vertical velocity) plumes originating from other sources in the downstream direction (due to the larger distance which separates them when $\eta$ is increased). 


\subsection{More turbulent regimes}

Having finished a description of the dynamics for $R a=\mathrm{O}\left(10^{8}\right)$ we now turn to discussing the analogous phenomena, which occur when the Rayleigh number is raised to $2.7 \times 10^{10}$. A first example for this case is shown in Fig. 15 for the same value of the Richardson number already considered in Fig. $5(\operatorname{Pr} R i=30)$.

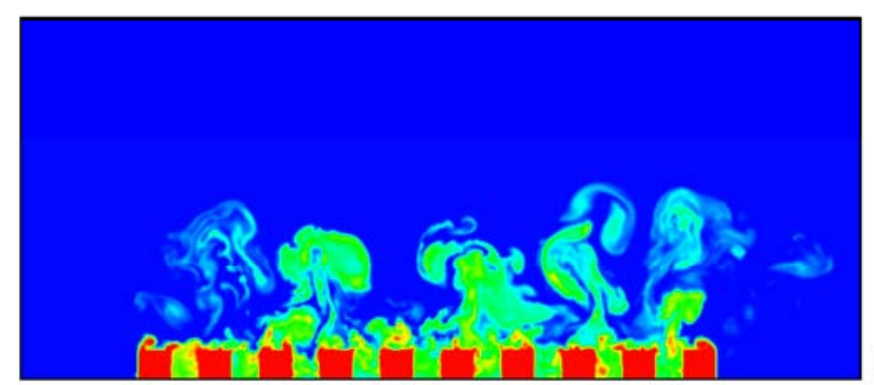

a)

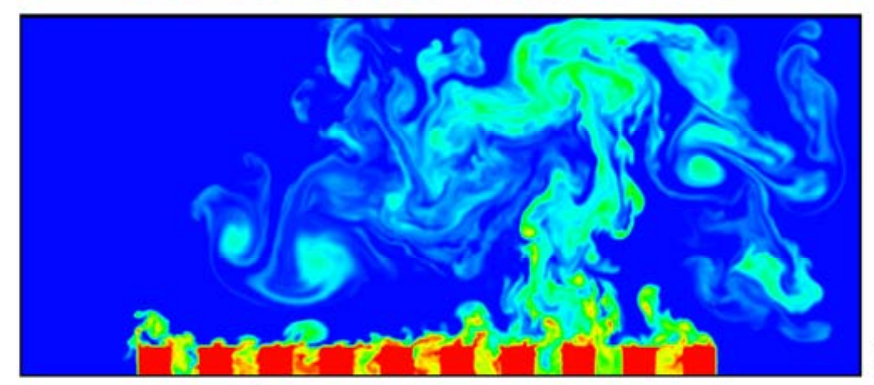

b)

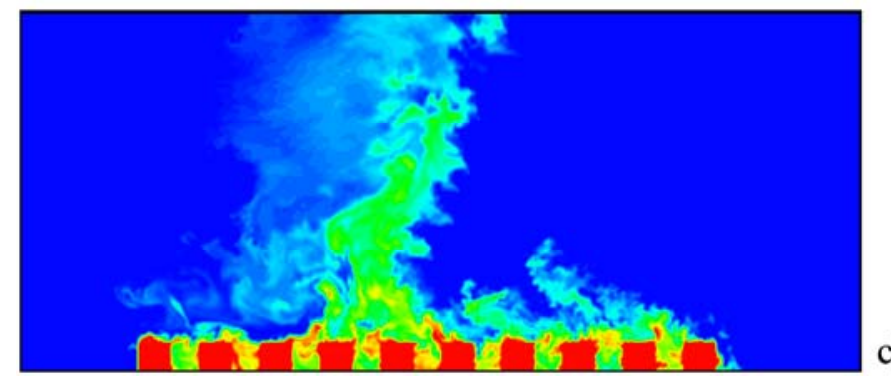

c)

Figure 15: Sequence of snapshots for the case $\left.R a=2.7 \times 10^{10}, \operatorname{Re}=3 \times 10^{4}, \operatorname{Pr} R i=30, \eta=1: a\right) t \cong 2.5, b$ ) $\mathrm{t} \cong 5, \mathrm{c}) \mathrm{t} \cong 10$.

Once again, these figures witness that for a relatively high value of $R i$, the emerging scenario is formally similar to that potentially supported by pure RB convection. Plumes being generated at different stations in different instants (asynchronous process) tend to accumulate at the center of the domain where they merge leading to the formation of a spatially extended rising current (giant plume). For continuity, fluid enters the domain from the right open boundary (Fig. 18). As already discussed in Sect. 4.2, the existence of such a self-produced horizontal 'wind' makes the problem of plumes originating from discrete sources of buoyancy in a cross flow at high $R i$ formally equivalent to that of turbulent RB. However, (as expected) for mixed buoyant-wind flow the large scale pillars generally display a slightly eccentric position with respect to the center of the domain (Figs. 15c and 16c). 


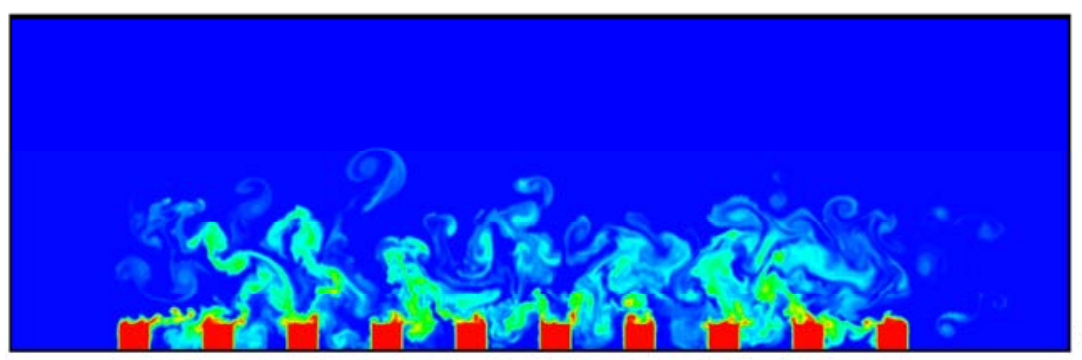

a)

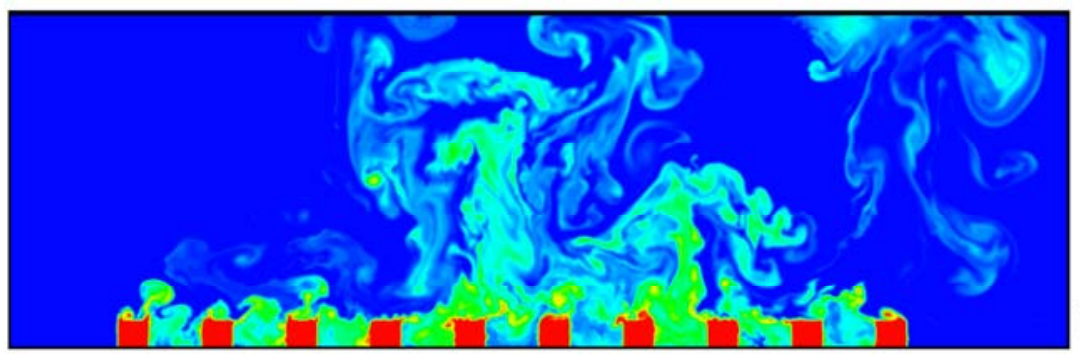

b)

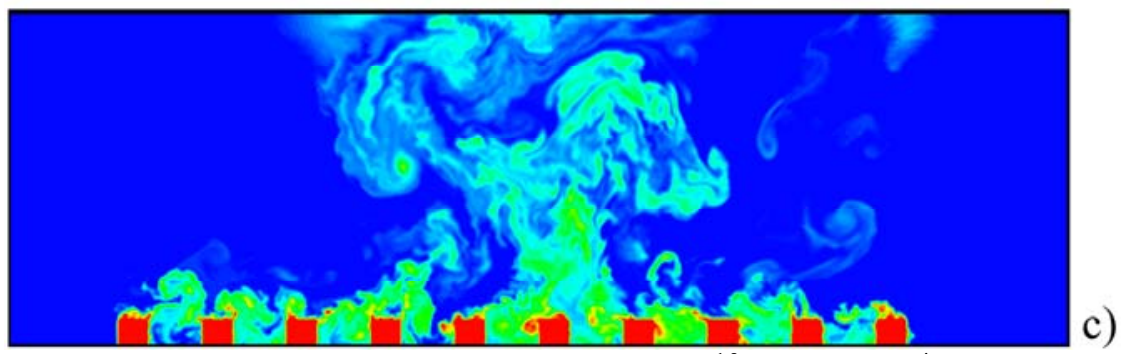

Figure 16: Sequence of snapshots for the case $R a=2.7 \times 10^{10}, \operatorname{Re}=3 \times 10^{4}, \operatorname{Pr} R i=30, \eta=2:$ a) $\mathrm{t} \cong 2.5$, b) $\mathrm{t} \cong 5, \mathrm{c}) \mathrm{t} \cong 7.5$.
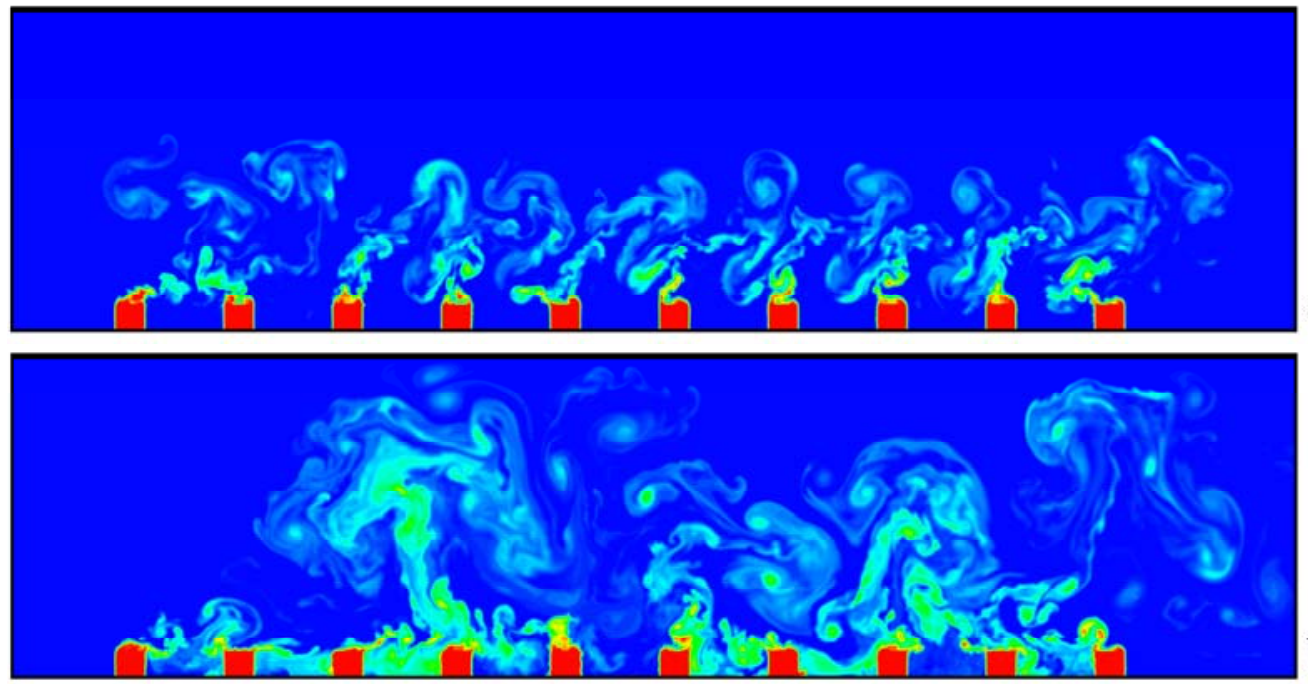

b)

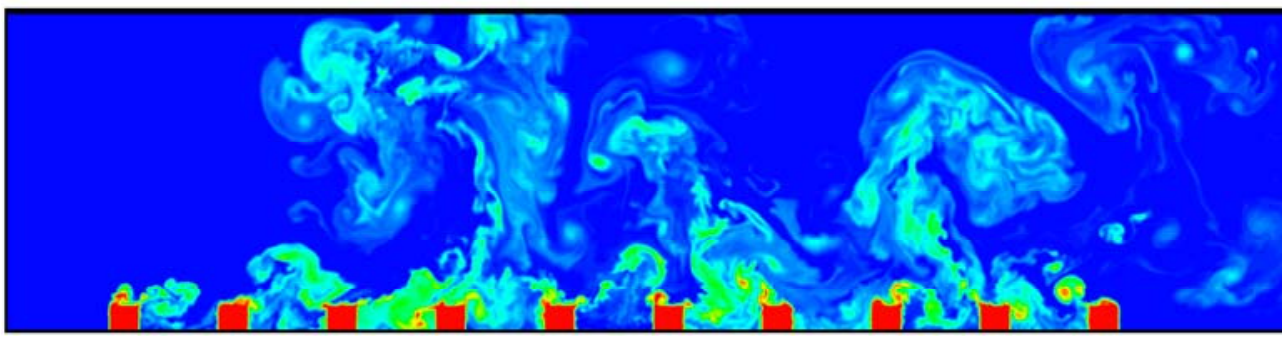

c)

Figure 17: Sequence of snapshots for the case $R a=2.7 \times 10^{10}, \operatorname{Re}=3 \times 10^{4}, \operatorname{Pr} R i=30, \eta=3$ : a) $\mathrm{t} \cong 2.5$, b) $\mathrm{t} \cong 5, \mathrm{c}) \mathrm{t} \cong 7.5$. 


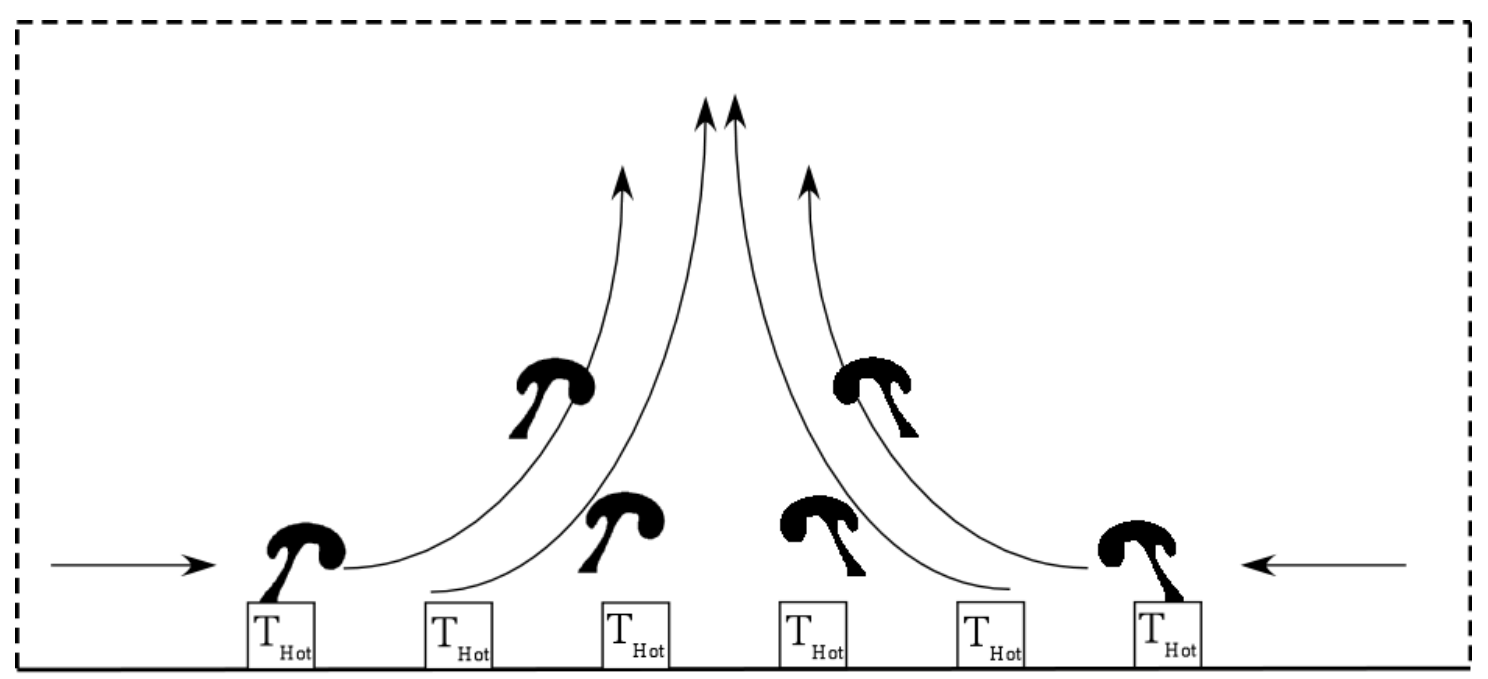

Figure 18: Sketch of large scale flow emerging for pure RB convection or mixed flow with large Ri.

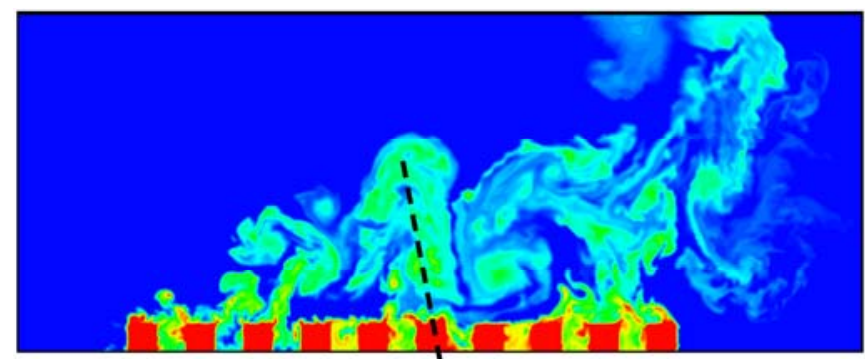

a)

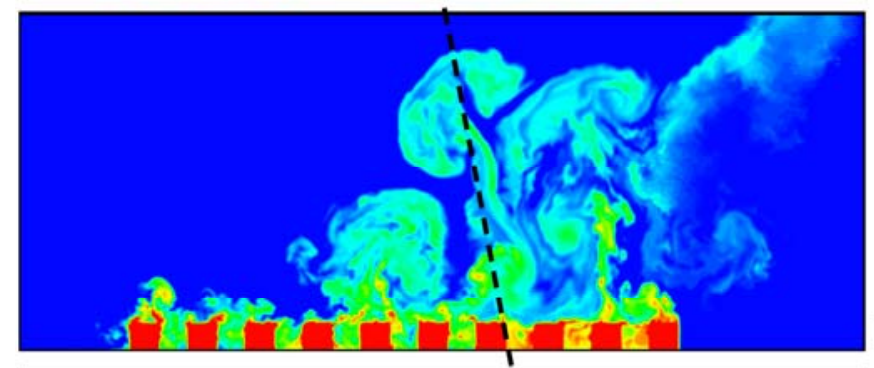

b)

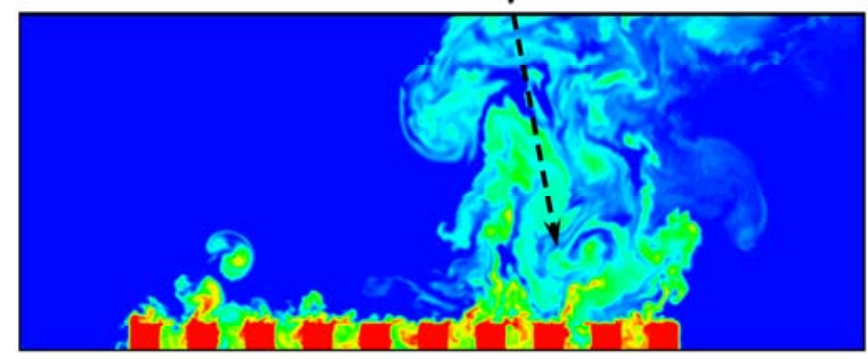

c)

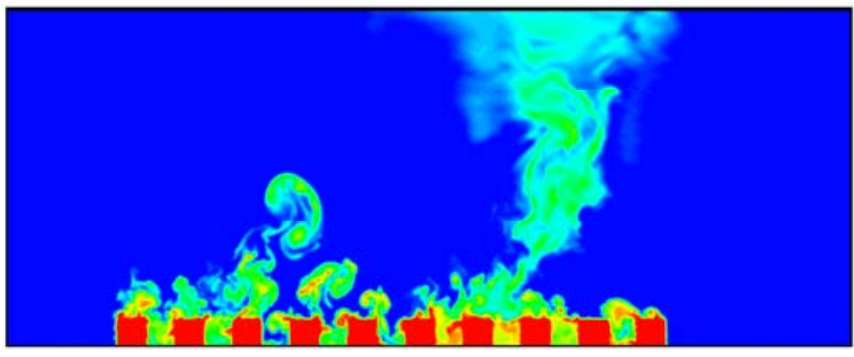

d)

Figure 19: Sequence of snapshots for the case $\left.R a=2.7 \times 10^{10}, \operatorname{Re}=7.5 \times 10^{4}, \operatorname{Pr} R i \cong 5, \eta=1: a\right) t \cong 75.6, b$ ) $\mathrm{t} \cong 75.9$, c) $\mathrm{t} \cong 78.2$. 


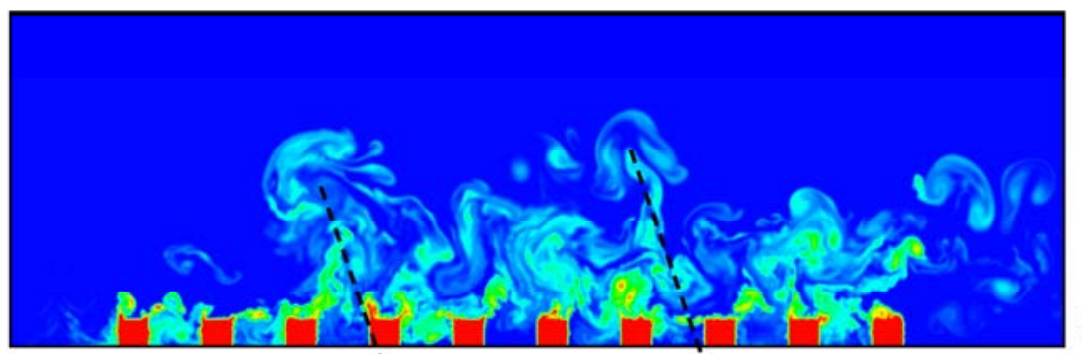

a)

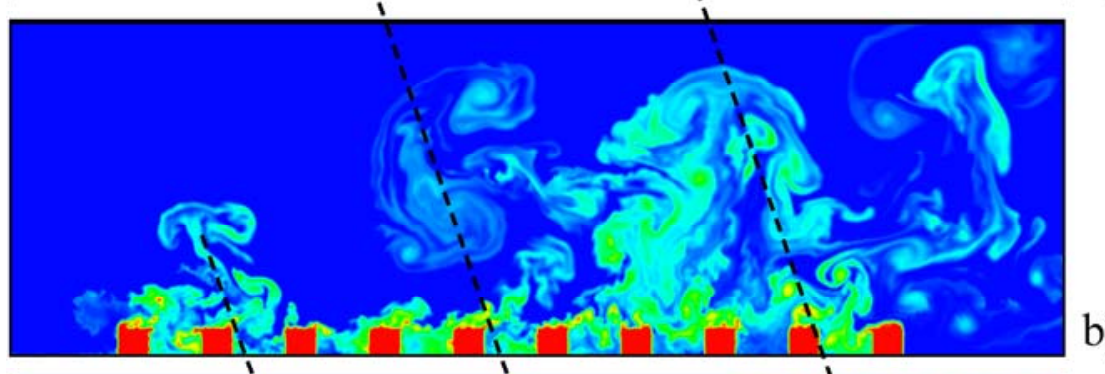

b)

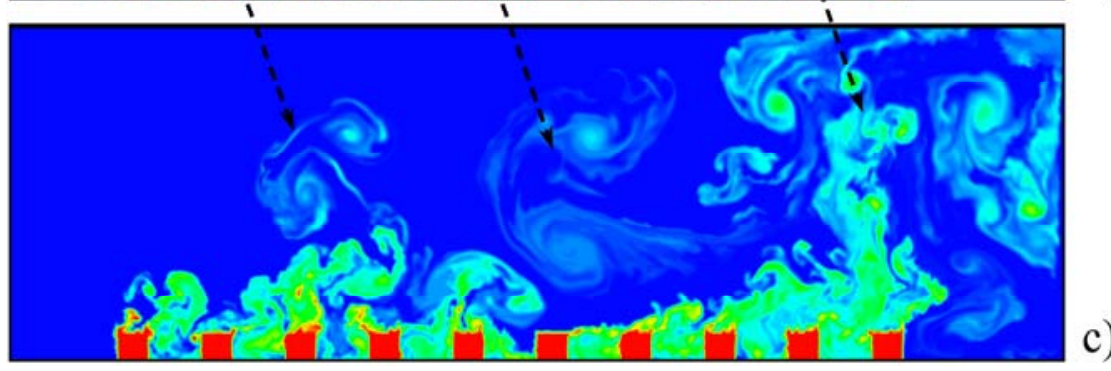

Figure 20: Sequence of snapshots for the case $\left.R a=2.7 \times 10^{10}, \operatorname{Re}=7.5 \times 10^{4}, \operatorname{Pr} R i \cong 5, \eta=2: a\right) t \cong 7.5, b$ ) $\mathrm{t} \cong 11.2, \mathrm{c}) \mathrm{t} \cong 15$.

Moreover, it can be seen that, in general, for this value of the Rayleigh number, smaller and more frequent eddies are thrown into the flow indicating strong interaction between the horizontal wind and the rising currents. While in equivalent internal flow problems where no buoyancy is present (see, e.g., Miyake et al., ${ }^{47-48}$ ), turbulators (ribs) break the laminar sublayer and give rise to localized wall turbulence due to flow separation and reattachment between the ribs, for the conditions under investigation here plumes are the main source of unsteady effects.

On decreasing PrRi to the same intermediate value considered for Figs. 9-11, a situation with unidirectional (rightward) horizontal wind is recovered (Figs. 19-21). The pillars formed by the plume coalescence mechanism are transported by such a wind. When a pillar leaves the domain crossing the right outflow boundary, a new plume aggregate has already started to grow at the left side thereby making the entire process somehow cyclic (endless process which repeats in time) and keeping constant the number $N$ of pillars present on average in the system $(\mathrm{N} \cong \eta$ as for the case with smaller value of the Rayleigh number, which may indicate that for a fixed value of the Richardson number $N$ depends only on the effective discrete distribution of heat sources considered). 


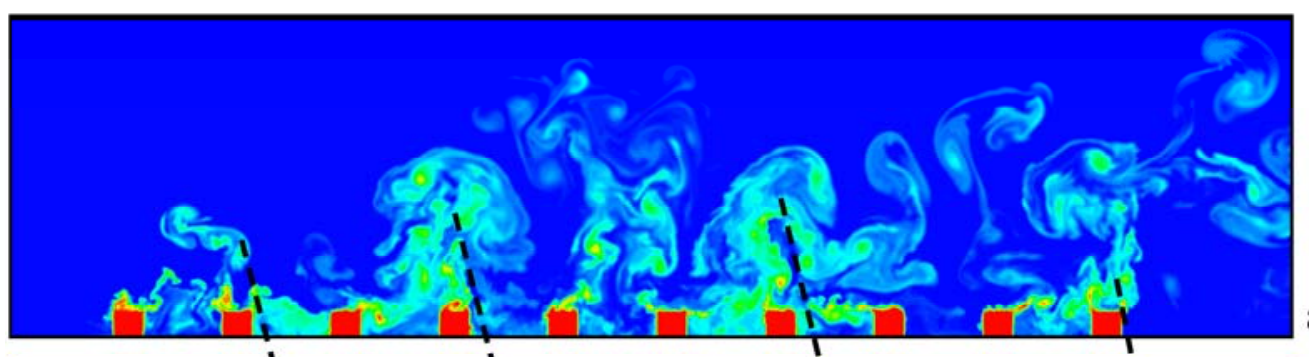

a)

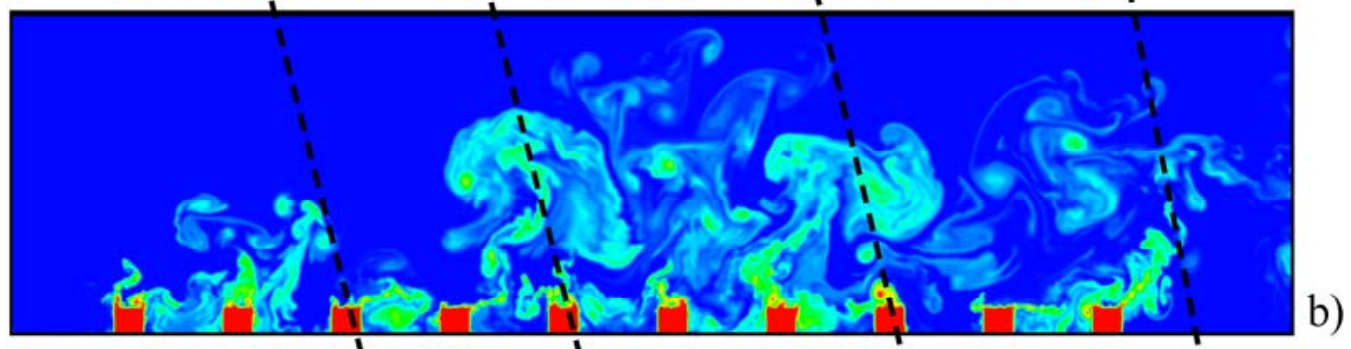

b)

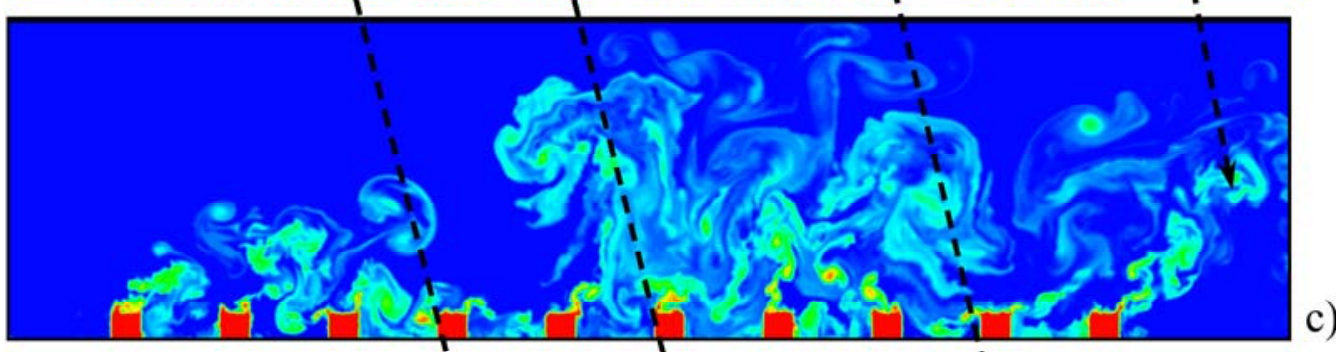

c)

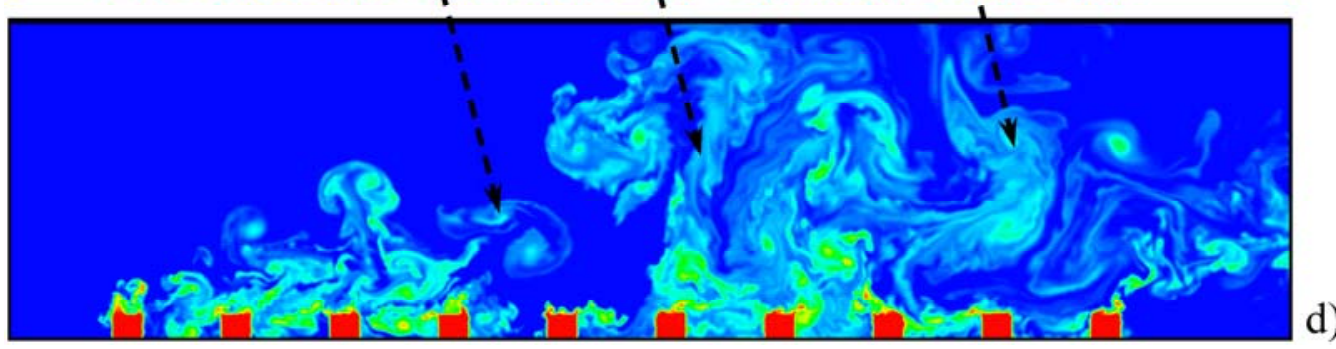

Figure 21: Sequence of snapshots for the case $\left.R a=2.7 \times 10^{10}, \operatorname{Re}=7.5 \times 10^{4}, \operatorname{Pr} R i \cong 5, \eta=3: a\right) t \cong 10.5, b$ ) $\mathrm{t} \cong 11.8, \mathrm{c}) \mathrm{t} \cong 13, \mathrm{~d}) \mathrm{t} \cong 14.3$.

\subsection{Hierarchy of scales and departure from the Kolmogorov law}

In the present section, a further understanding of the observed dynamics is gained by considering a fine-grained micromechanical perspective, which helps us to uncover some differences among the different considered cases (not revealed by the macrophysical approach used in Sect. 4.4). In order to clarify the 'nature' of the examined turbulent states, in general, a slightly different point of view must be taken, that is, leaving aside for a while the strong nonlinear nature of macroscopic evolution, one has to deal with issues of complex behavior at smaller scales, where an interesting hierarchy of phenomena is established. In particular, the next level along such a hierarchy of scales is to take directly a close look at the dynamics of the eddies present in the flow (which, by interacting lead to the emergent macroscopic properties discussed before). 
As a fleeting glimpse into Fig. 22 would confirm, the spatio-temporal evolution of the system for $R a=\mathrm{O}\left(10^{10}\right)$ becomes much more complex (the reader, e.g., may compare directly Fig. 22 and Fig. $13 \mathrm{a}$ for the same value of the Richardson number and $\eta$ ).

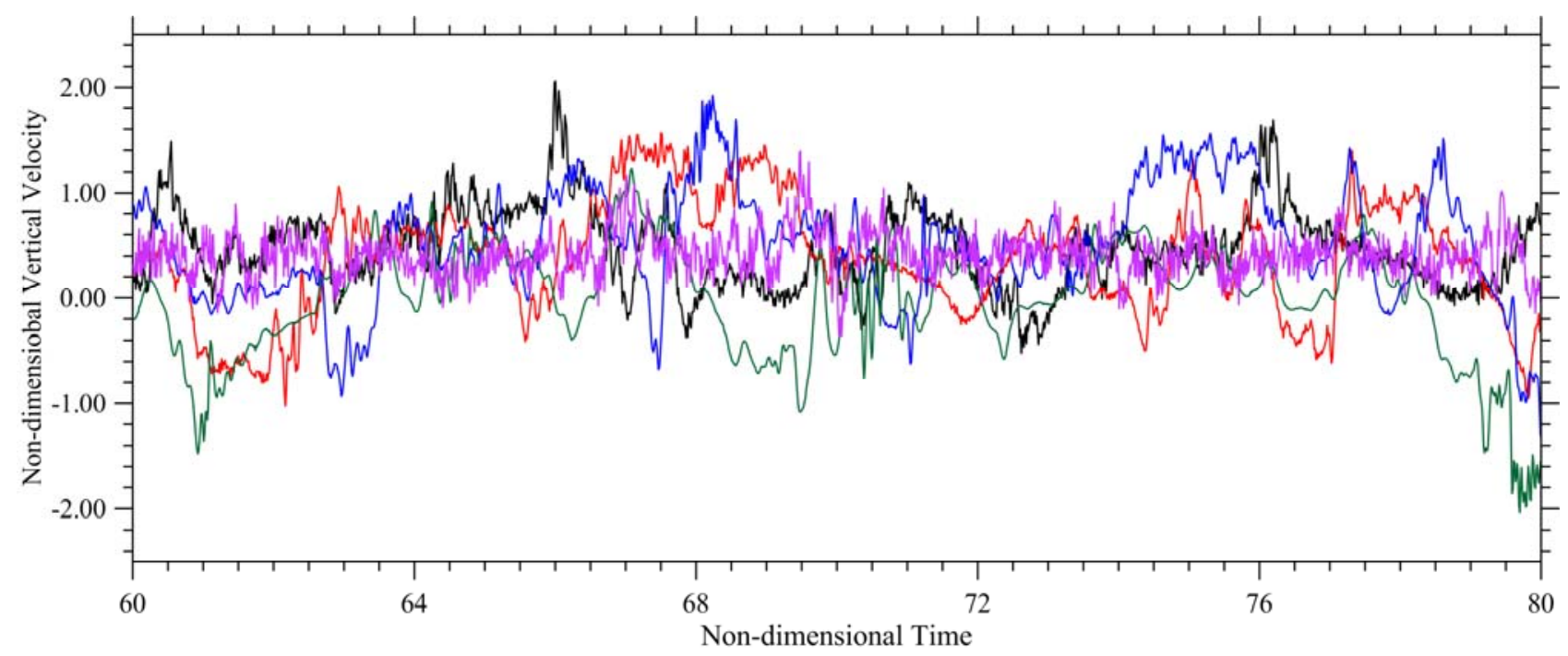

Figure 22: Signals provided by probes located above each heated item (purple, black, red, blue, green lines corresponding to probes $1,3,5,7,9$, respectively) for $R a=2.7 \times 10^{10}, R e=7.5 \times 10^{4}$ and $\eta=1$.

To elucidate further the significance of this observation, one should keep in mind that, even if the Richardson number may be regarded as the most influential parameter in terms of general features of the pattern and related evolutionary progress, the problem still retains a strong dependence on the effective values of the Rayleigh and Reynolds numbers. Indeed, an increase in these parameters, while keeping fixed Ri, has a two-fold effect. When the Rayleigh number is made higher, as already explained before, the frequency of production of plumes undergoes a remarkable increase (as it is also the case of standard RB convection). At the same time, the intensity of horizontal shear also grows, thereby making plume stems more susceptible to shear driven instabilities that can cause plume stalk deformation and plume cap detachment. On the other hand, an increase in Re, can cause plume instability due to the rise in the vertical shear. In practice, both mechanisms concur in determining plume splitting phenomena.

As a result (the reader being referred again to Figs. 19-21), the size of eddies visible in the temperature and velocity fields decreases significantly, this effect being associated with a significant rise in the typical frequency of signals measured by probes at fixed locations (Fig. 22).

Additional insights into this scenario can be gathered from an analysis of the typical frequency spectra, which have been reported in Fig. 23 for the same signals shown in Fig. $22\left(R a=2.7 \times 10^{10}\right.$, $\mathrm{Re}=7.5 \times 10^{4}$ and $\left.\eta=1\right)$. 

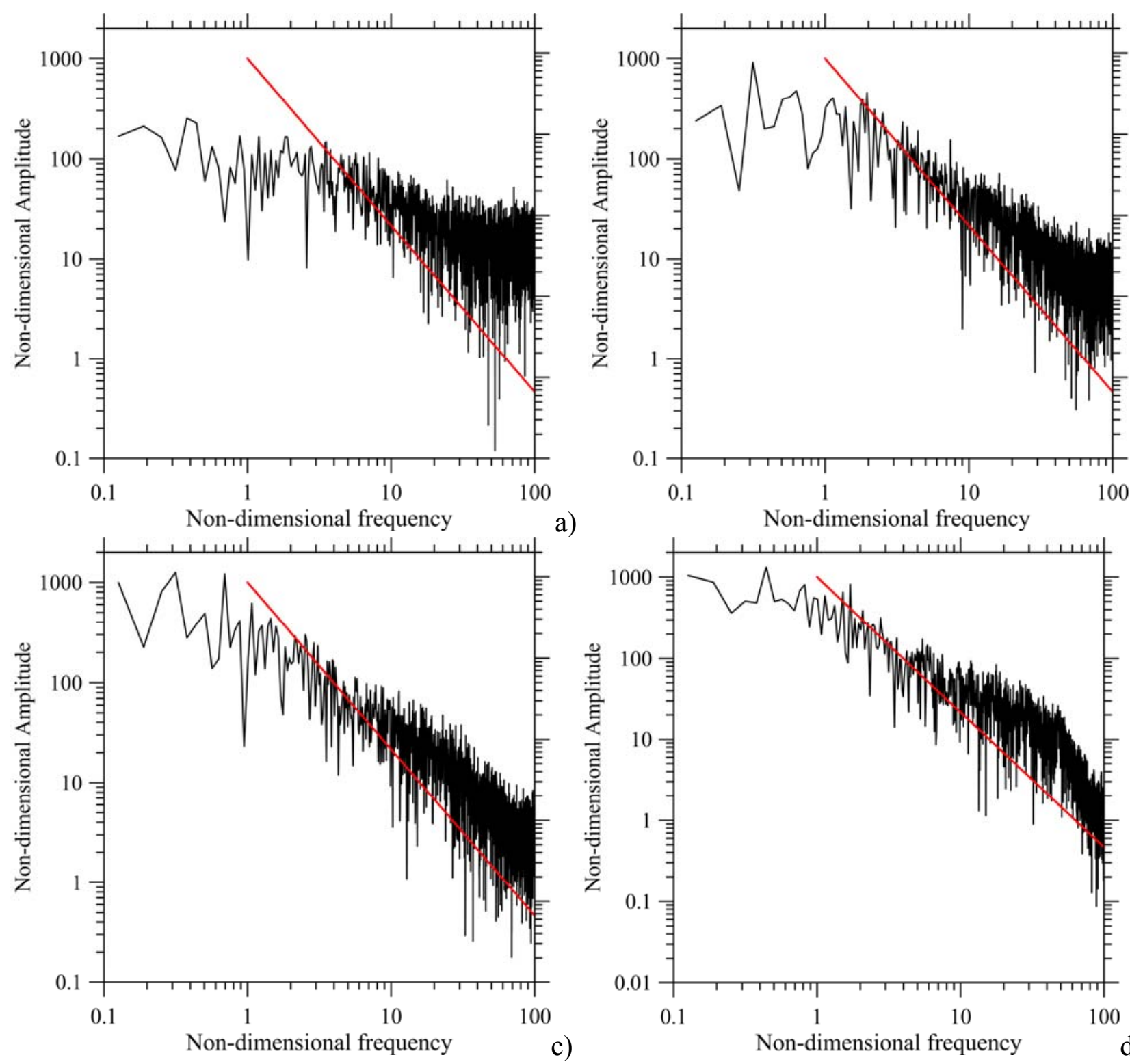

a)

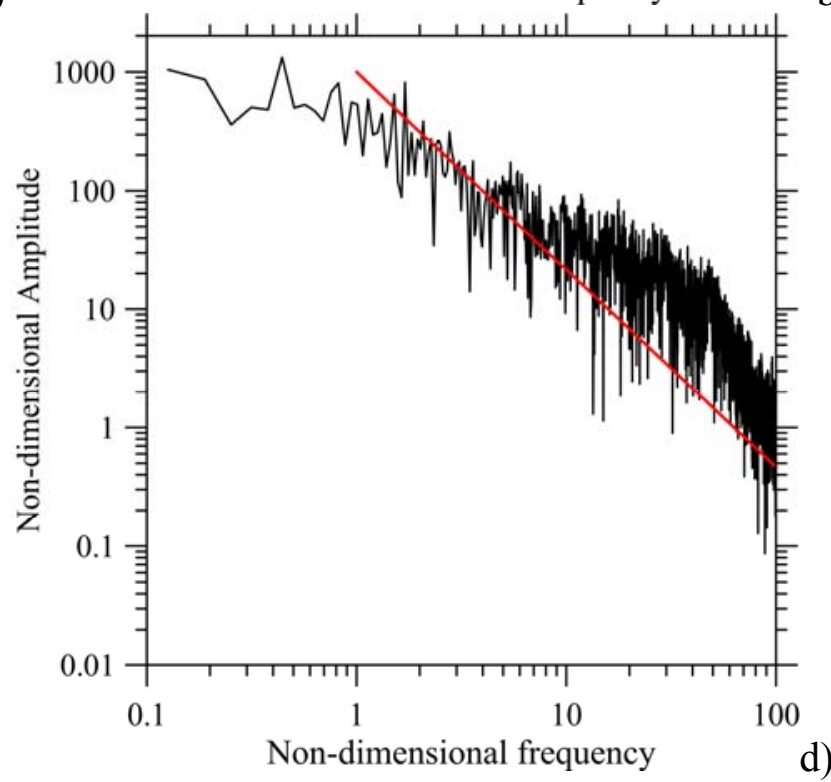

Figure 23: Frequency spectra for $R a=2.7 \times 10^{10}, \mathrm{Re}=7.5 \times 10^{4}$ and $\eta=1$ : a) probe 1 , b) probe 3 , c) probe $5, d$ ) probe 7 . The spectrum relating to probe 9 has not been included as it is almost identical to that for the probe 9. All the plots have been truncated at $\omega=100$. Consideration of these spectra at larger frequencies would make no sense as these frequencies are not directly resolved by LES (which implicitly account for them via the Smagorinsky-Lilly model).

The high-dimensional nature of the chaos relating to the state visible in Fig. 22 is confirmed by the agreement of the spectra with the behavior that one would expect on the basis of the theory by Kolmogorov ${ }^{77-80}$ about the development of isotropic turbulence (already discussed to a certain extent in Sect. 2.2). In order to make this specific aspect evident, following typical practice in the literature (see, e.g., De et al., ${ }^{98}$; Lappa and Gradiscak ${ }^{120}$; Oskouie et al., ${ }^{121}$ ), we have reported the frequency and related amplitude in these figures using logarithmic scales. Moreover, we have also plotted a power law $\mathrm{P}(\omega)=\left(\omega / \omega_{\mathrm{c}}\right)^{-5 / 3}$ (where $\omega_{\mathrm{c}}$ is a fitting parameter), just to help the reader to verify that, as predicted by the Kolmogorov's turbulence similarity hypothesis, the frequency spectrum of velocities aligns with the $\omega^{-5 / 3}$ law in an extended range of frequencies. 
Along these lines, it is worth recalling that Kolmogorov (Kraichnan ${ }^{82}$ ) originally elaborated a picture of steady, homogeneous, isotropic turbulence in which equilibrium conditions are attained because the energy injected in the flow per unit time is balanced precisely by the amount of energy dissipated per unit time. Such dissipation is due to molecular viscosity and, therefore, takes place at very short length scales while the injection occurs at macroscopic length scales. Indicating by $\varepsilon$ the common rate of injection and dissipation, according to this theory the energy cascades tightly at this (constant) rate from the large scales of injection to the short scales of dissipation, this process being mediated by the non-linear terms in the governing Navier-Stokes equation. In particular, the most interesting aspect of this theory is that an intermediate range of wavenumbers $(k)$ exists, which is well separated from large $\mathrm{k}$ (corresponding to small length scales) and also from small $\mathrm{k}$ (corresponding to large length scale), where the energy spectrum is not influenced by the specific mechanisms of energy input and loss and depends only on $\varepsilon$ and the local wave-number $\mathrm{k}$ (a dimensional analysis leading to $\left.\mathrm{E}(\mathrm{k}) \propto \mathrm{k}^{-5 / 3}\right)$.

From a practical standpoint, such an interval is limited at one side by the a length-scale $\cong H$ representative of the considered domain, which is the scale at which energy is injected into the fluid, and at the other side by the scale where internal energy is produced by frictional effects at expenses of the kinetic energy of the flow. In the present case, we have truncated the plots at $\omega=100$ as the simulation was no longer able to capture convective modes with frequencies lying above this threshold (the effect of these high wavenumber spatial modes, however, was implicitly taken into account in the framework of the approach described in Sects. 2.3-2.4, that is the intrinsic philosophy on which LES modes are based).

Interestingly, though all spectra follow with a good degree of approximation the Kolmogorov law, some weak departure can be seen for the behavior of the first two or three probes where the average inclination of the spectrum seems to be weaker. Since for $R a=2.7 \times 10^{10}$ and smaller value of Re $(\operatorname{Pr} R i=30)$ we found the slope of all the spectra to align with the Kolmogorov law regardless of the considered station (not shown), we conclude such a departure is essentially due to the mechanism of plume removal (described in Sect. 4.3) being operative there, which, by interfering with the growth and detachment of plumes, causes a modification in the behavior of turbulence driven by thermal buoyancy.

The trend remains essentially the same if another (larger) value of the spacing parameter $\eta$ is considered (see, e.g., Fig. 24 for $\eta=3$ ). As shown in this figure, for $\eta=3$ the appearance of the spectra (in terms of tide) is yet non-homogeneous, as they displays appreciable modifications weakly depending on the sequential order of probe position. However, in this case, interestingly, the spectra are simpler, which can be ascribed to the reduced number of plumes being present in the domain at a fixed time per unit length when the spacing among the sources is increased. 

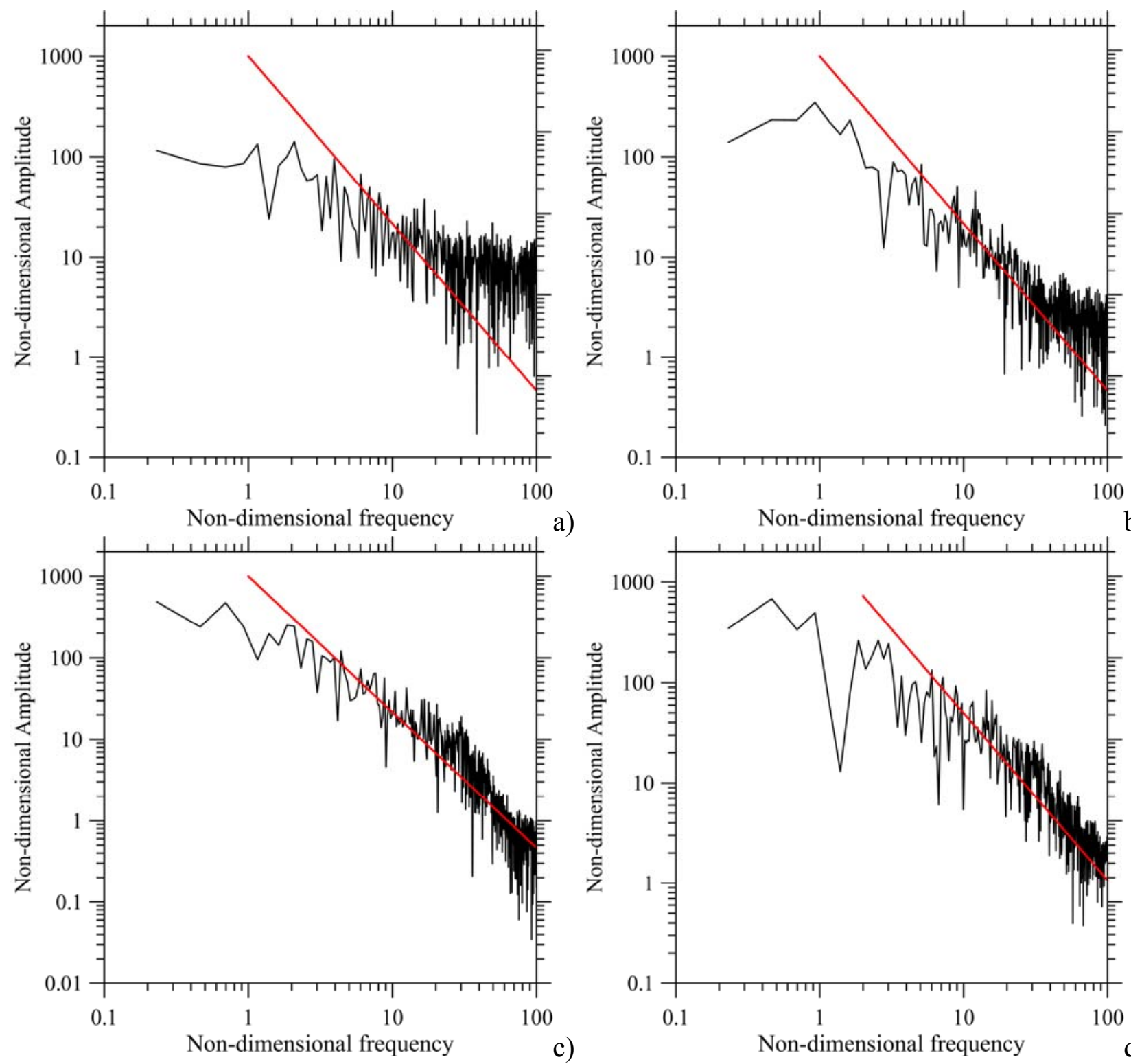

a)

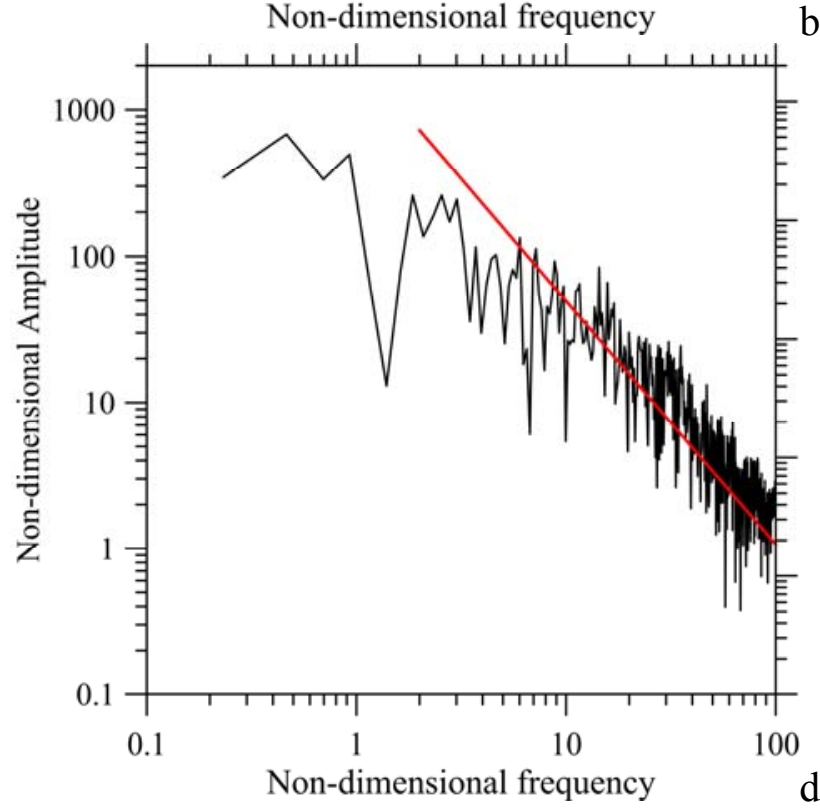

Figure 24: Frequency spectra for $R a=2.7 \times 10^{10}, \mathrm{Re}=7.5 \times 10^{4}$ and $\eta=3$ : a) probe 1 , b) probe 3 , c) probe $5, \mathrm{~d})$ probe 7 .

\subsection{Sheltering effect at high Ra and "bubble" formation}

Notably, the descriptions given in the earlier section about the dependence of plume dynamics on the considered station (together with the observed different spatio-temporal behavior relating to the first heat source) may be vaguely reminiscent of an effect already known for the case of jets interacting with a cross flow for conditions in which the overall flow is steady in mean, that is, the so-called "sheltering effect". Past analyses on this specific case have indeed revealed some anisotropy in the macroscopic patterning behavior. As illustrated, e.g., by Yu et al., ${ }^{122}$ and Lai and $\mathrm{Lee}^{26}$, given a set of jets in a cross-flow with dynamics steady in mean, the leading jet is typically deflected more by the ambient current, while the other (rear) jets are less affected (but all to a similar degree). The downstream jets, all having similar trajectories, typically experience a reduced 
effective crossflow velocity due to the blockage and sheltering effect of the leading jet ${ }^{26,122}$. There is some consensus in the literature that the shrinkage in the effective cross flow velocity for the rear jets becomes stronger when the jet spacing is decreased (however, it has been observed to depend weakly on the sequential order of jet position and the number of jets in the group).

Some analogies can be identified between these observations and the dynamics presented here. Even if the present plumes display a highly unsteady behavior, indeed, some kind of sheltering effects can yet be discerned with regard to the plumes originating from sources located at a certain distance from the first source of the succession. As discussed before, the convective structures produced by the coalescence of plumes apparently prevent the other plumes located downstream from interacting directly with the incoming horizontal wind. This effect allows plumes located downstream to develop and extend along the vertical extension to an appreciable extent (though affected by instability and swaying motion) before their stem is broken and/or cap detachment occurs. Some hierarchy can also be identified in terms of spectra. These display inclination slightly depending on the relative position of the considered source until they become all similar (and follow the Kolmogorov law just like plumes in RB convection would do) beyond a given station (which, in turn, we find to be a function of the spacing parameter $\eta$ ).

What is emphasized here is the possible inter-relatedness between the anisotropy in the frequency spectra behavior as a function of the position and the purely geometrical effect identified in past analyses for flows that are essentially steady in mean.

All these arguments may be repeated under a slightly different perspective by simply stating that while the classical sheltering effect manifests essentially in terms of geometrical features (steady plumes located downstream less deflected), it is somehow 'transferred' to the temporal features of the system when turbulent systems (such as those examined here) are considered. In our case, obviously, there is no (more deflected) plume stably located at the first station. Rather, as illustrated before, there the dynamics are dominated by the continuous interference of vertical shear (horizontal wind) and buoyancy (horizontal shear), as witnessed by the high frequency of local oscillation and their relatively small amplitude (see Sect. 4.3).

As a concluding remark for this analysis, we wish to highlight that, interestingly, the simulations performed for $R a=2.7 \times 10^{10}$ have revealed that the dynamics established in a given neighborhood of the first station might be even richer than those illustrated in Sect. 4.3 if relatively high values of the Reynolds number are considered. 


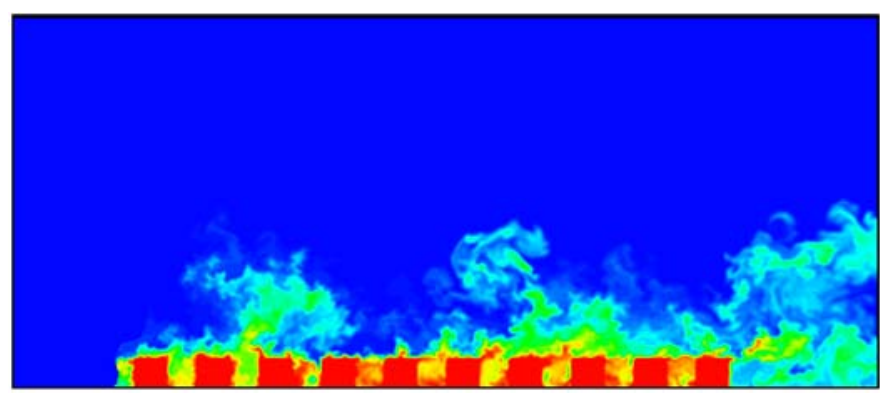

Figure 25: Snapshots for the case $R a=2.7 \times 10^{10}, \operatorname{Re}=3 \times 10^{5}, \operatorname{Pr} R i=0.3, \eta=1, \mathrm{t} \cong 50$.

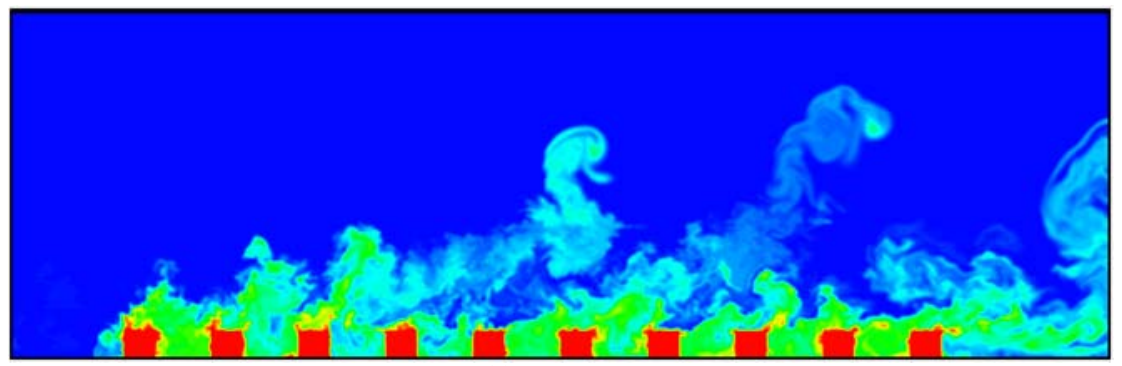

Figure 26: Snapshots for the case $R a=2.7 \times 10^{10}, \operatorname{Re}=3 \times 10^{5}, \operatorname{Pr} R i=0.3, \eta=2, \mathrm{t} \cong 45$.

For $\mathrm{Re}=3 \times 10^{5}(\mathrm{Pr} R i=0.3$, Figs. 25-27), the maximum vertical extension of the plume aggregates is relatively limited. For this value of $R i$, moreover, though large plumes resulting from the coalescence of eddies can be found in the right part of the domain, plumes tend to maintain the small-scale structure that they have when they are formed and detach from the top of the square heat sources (Fig. 25-26). Nevertheless, for $\eta=2$ and $\eta=3$ a localized recirculation with relatively large scale can be seen across the entire region in between the first and second source (this is very evident, e.g., in Fig. 27). This recirculation (horizontally extended above the first two sources) entraps heat, thereby leading to a local temperature that is slightly higher than the temperature established between other couples of heated elements. As shown in Fig. 27, sometimes this recirculation (hereafter we will refer to it as the heat 'bubble') breaks and releases heat to the external environment in the form of a localized 'eruption', i.e. a short-lived vigorous vertical movement, which creates locally a rising column of warm fluid that is then bended by the horizontal wind and forced to reattach (this behavior being reflected by the vigorous oscillations measured by the first numerical probe, see Fig. 28, which might be regarded as a clear distinguishing mark with respect to the behavior shown in Fig. 22).

Bubbles of such a kind are relatively common when a wind with relatively high value of the Reynolds number interacts with a topographic obstacle (see, e.g., Mason and Sykes, ${ }^{123}$ ) or inside isothermal channels where the flow meets a forward-facing step (Largeau and Moriniere ${ }^{124}$; Abdalla et al., ${ }^{125}$; Hattori and Nagano ${ }^{126}$ ). The present simulations reveal that bubble interaction with the inherently unsteady dynamics of thermal plumes can lead to a new kind of effect. 


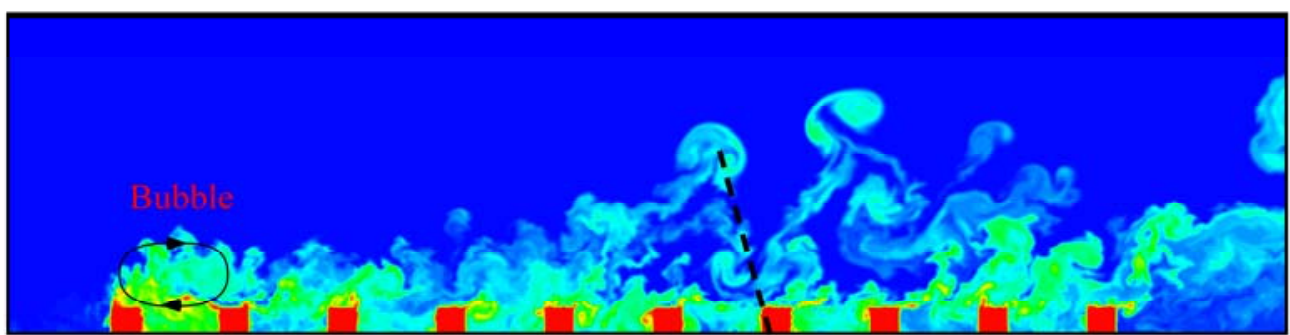

a)

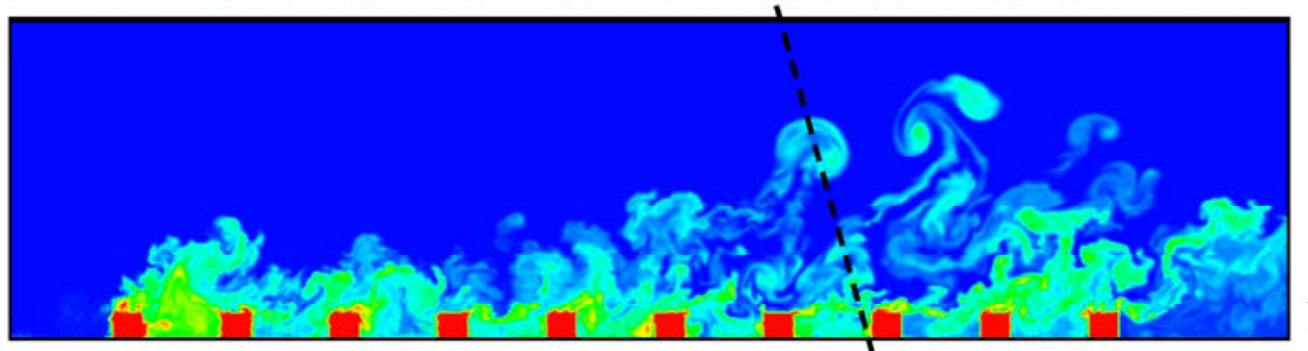

b)

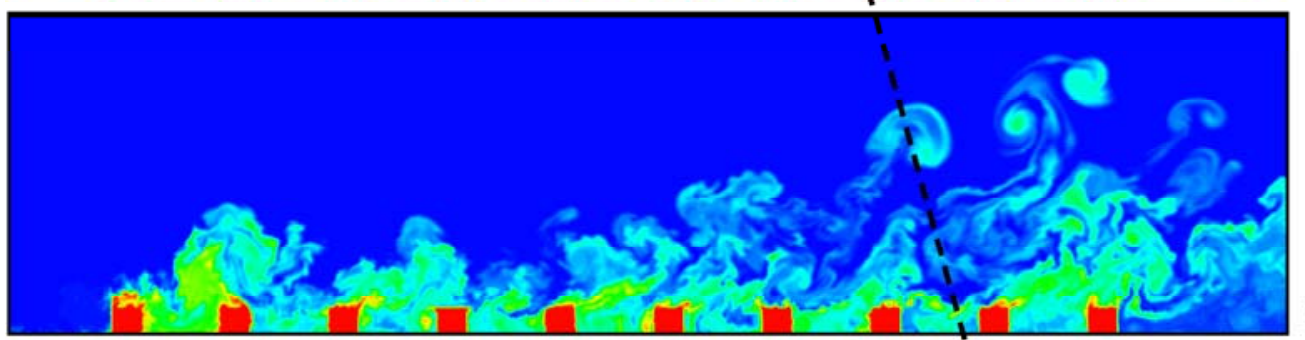

c)

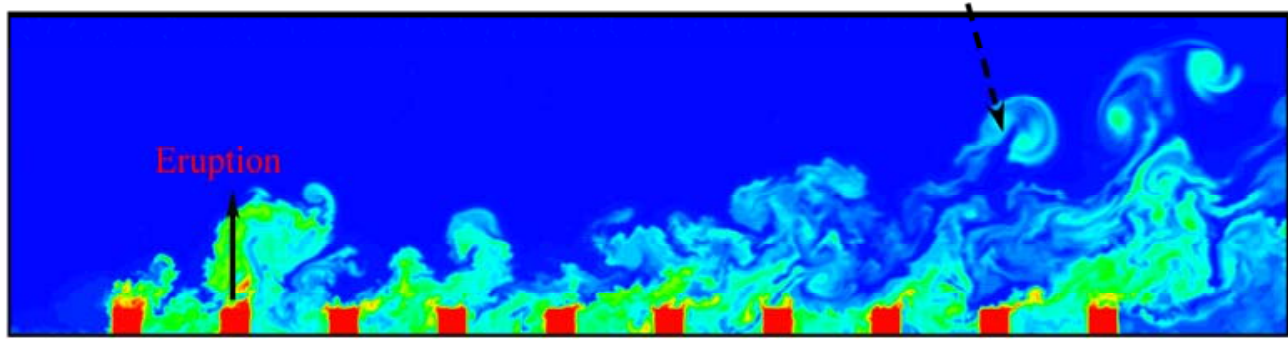

d)

Figure 27: Snapshots for the case $R a=2.7 \times 10^{10}, \operatorname{Re}=3 \times 10^{5}, \operatorname{Pr} R i=0.3, \eta=3$, initial time $=55$, $\Delta$ time $=1.5$, a recirculation is visible between the first and second sources while large vortical structures resulting from the coalescence of eddies can be seen in the right part of the domain.

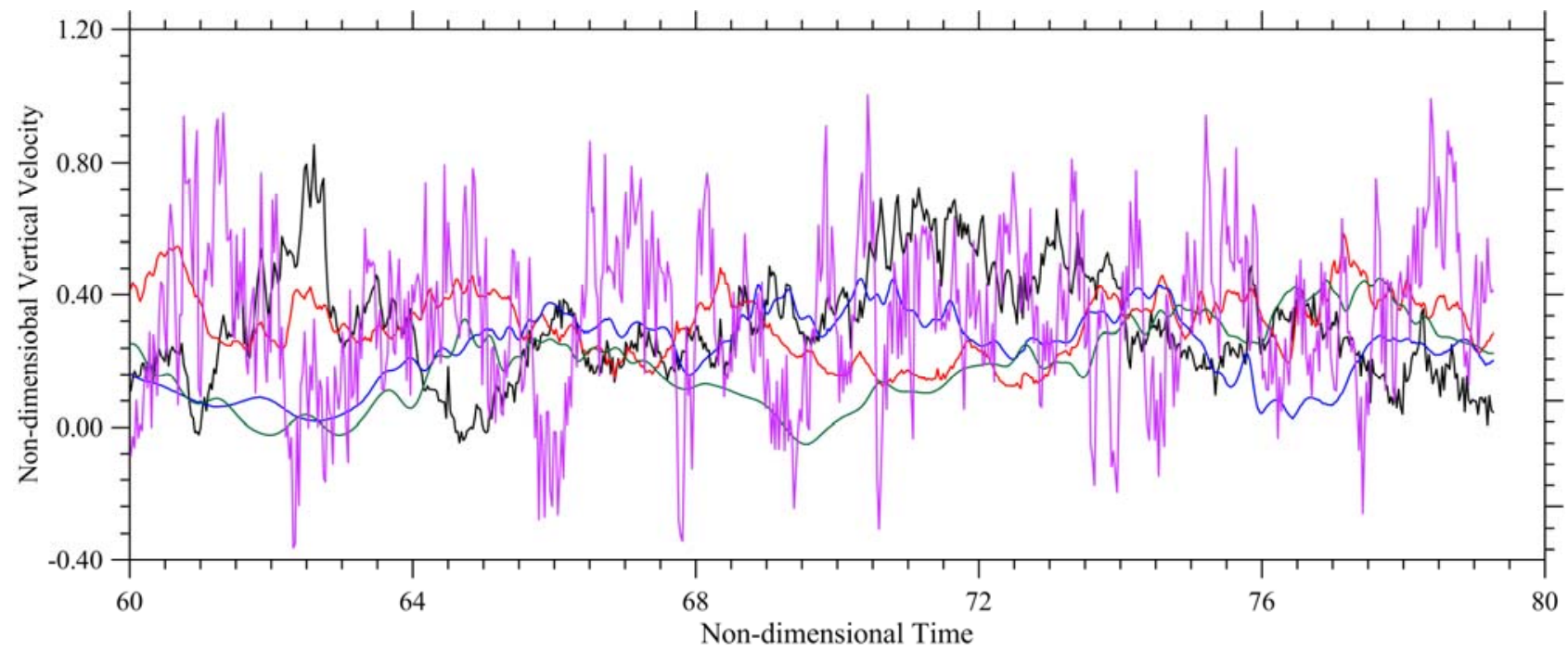

Figure 28: Signals provided by probes located above each heated item (purple, black, red, blue, green lines corresponding to probes $1,3,5,7,9$, respectively) for $R a=2.7 \times 10^{10}, \operatorname{Re}=3 \times 10^{5}$ and $\eta=3$. 

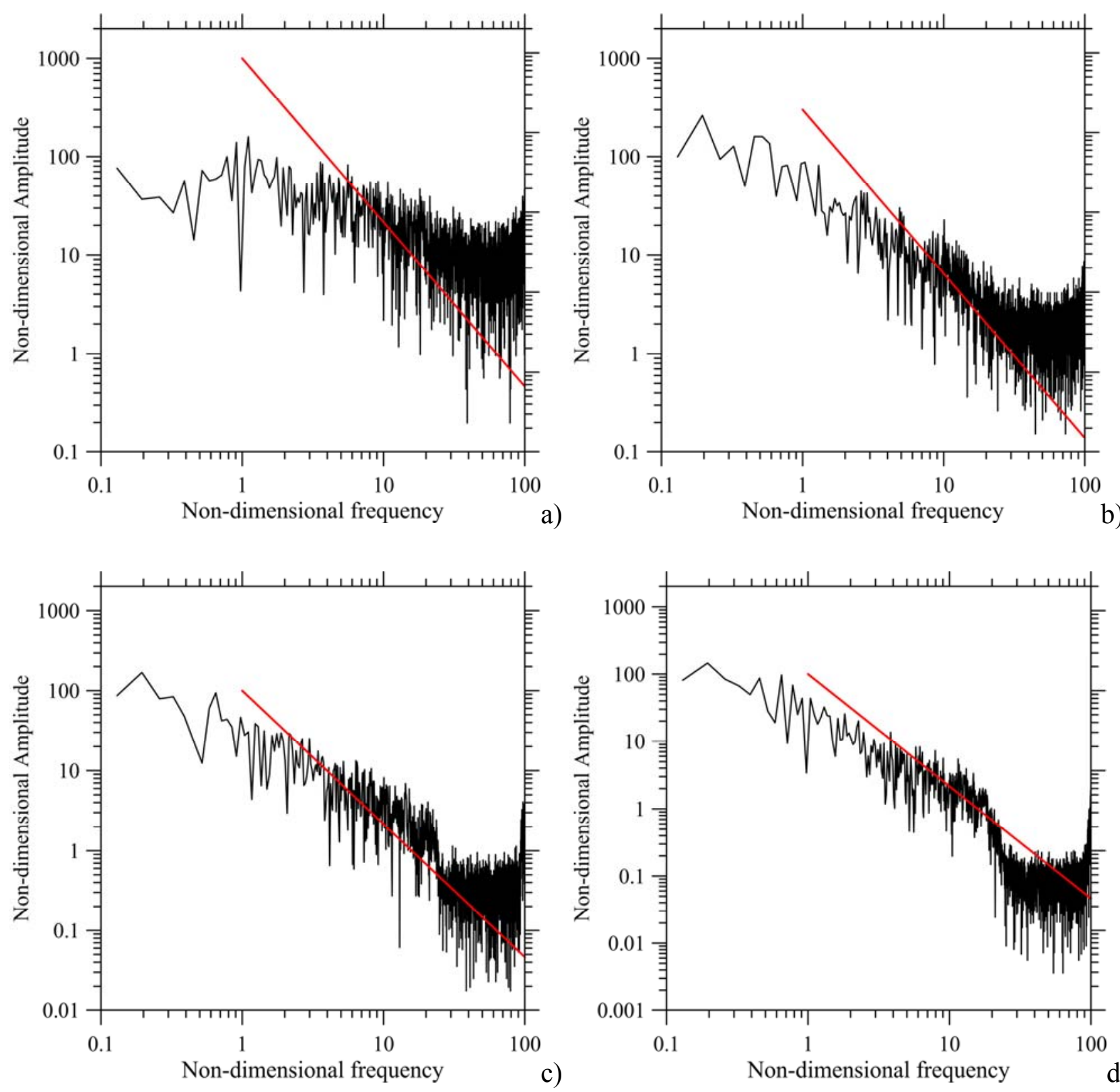

Figure 29: Frequency spectra for $R a=2.7 \times 10^{10}, \mathrm{Re}=3 \times 10^{5}$ and $\eta=3$ : a) probe $1, \mathrm{~b}$ ) probe 3 , c) probe 5 , d) probe 7 .

Most interestingly, as witnessed by the increased density of the spectrum in Fig. 29a with respect to that shown in Fig. 24a, the vigorous vertical movement created by the heat bubble rupture induced by thermal buoyancy, compensates the effects produced by the reduced number of plumes being present in the domain per unit length when the spacing among the sources is increased. Comparison with Fig. 24 also leads to the interesting conclusion that (as expected) for higher Re, a larger distance from the inflow section must be considered before the frequency spectrum aligns with the Kolmogorov law. 


\section{Conclusions}

Thermal plumes interacting with a cross flow (horizontal wind) represent a fundamental problem of great interest, standing at the intersection of many technological fields. Beyond practical applications, fascination with the spatiotemporal patterns that arise spontaneously in these nonequilibrium systems has stimulated detailed experimental and theoretical investigations resulting in a sound understanding of many aspects of the formation of related patterns. Despite the umbrella of on-going research on the subject, however, a large number of key issues and problems remain unresolved.

In the present study, starting from considerations relating to the so-called sheltering effect, that is, the known spatial (geometrical) anisotropy created in a group of thermal plumes by the interaction with the cross flow, we have elaborated a 'minimal' model to inquiry about the potential reverberations of this mechanism (known to be operative for cases in which plume trajectories do not depend on time) on flows which are extremely time dependent (turbulent).

Special care has been devoted to the assessment of the conditions which make the problem well posed from a computational point of view. Such assessment has been partially based on physical reasoning involving the known behavior of turbulence on certain scales on the one hand and on available estimates of such scales on the other hand. In particular, specific effort has been provided to investigate the triadic relationship among the different (typical) effects which determine the response of these systems, namely the spacing among sources, the intensity of buoyancy forces (measured by the Rayleigh number) and forced convection (governed by the Reynolds number) and identify possible universality classes of behaviors.

As revealed by the present simulations, the dynamics are rich and can produce different scenarios depending on the relative importance of the considered influential factors.

For sufficiently small value of the Reynolds number, the dynamics are formally similar to those of pure buoyancy convection. The main outcome of the plume coalescence process is a central thermal rising current. As a result of the significant amount of fluid leaving the physical domain through the upper boundary, by continuity new fluid is absorbed from both the left and right neighboring regions, producing as a natural consequence centerward directed horizontal currents (entering the domain from both the left and right sides). These winds further reinforce the mechanism leading thermal plumes originating from peripheral regions to cluster at the center thereby feeding the central pillar.

The (approximate) central symmetry of this configuration is obviously broken by the presence of the horizontal wind which interferes in different ways with the plume clustering process. More precisely, while buoyancy promotes the emergence of plumes and associated localized vorticity effects (horizontal shear), which lead to the spontaneous formation of large scale structures (in the form of vertically extended columnar regions of hot fluid), imposed wind (vertical shear) counteracts such tendency through a two-fold cause-and-effect mechanism: On the one hand it opposes to the natural coalescence process responsible for the formation of the large structures by 
promoting plume instabilities and splitting, on the other hand, it causes a continuous migration of the existing structures in the downstream direction. This effect prevents the formation of a single (static) thermal giant rising jet originating from the center of the configuration (which is replaced by "pillars" with intermediate size, dynamically travelling in the downstream direction).

The number of these features depends essentially on the heat source spacing (such spacing having obviously an impact on the distance that plumes originating at different locations have to travel before they can coalesce) and on the value taken by the Reynolds number. In particular, as Re grows, the number of pillars increases while their size becomes smaller. Since the wind prevents many small scale plumes from travelling in the upstream direction, for these cases most of pillar growth occurs essentially because the convective structures resulting from localized coalescence process travel in the downstream direction (thereby capturing newborn plumes along their way).

The departure from the situation which would be produced by pure buoyancy convection is not limited to the patterning behavior displayed by plumes and the spatial aggregation phenomena discussed above. It also occurs on a range of smaller scales, as made evident by the frequency spectra related to velocity signals taken at fixed (evenly spaced) positions. For the probes located in proximity to the inflow section (in correspondence of the first two or three heat sources) the average trend (inclination) of the frequency spectrum is slightly smaller than that predicted by the Kolmogorov similarity hypothesis, whereas the agreement is very good for stations located at a sufficient distance from that section. On the basis of the numerical simulations, we conclude that such a departure must be ascribed to a fast mechanism of plume removal/rebirth that is established in proximity to the inflow section. As soon as a plume is spread along the horizontal direction by the imposed wind and relatively warm fluid is replaced by cold fluid, a new plume is formed at that station. As a result, high-frequency components enter the velocity spectrum of frequencies. These components vanish when probes located further in the downstream direction are examined. This effect can be explained taking into account that the development of plumes originating from the top surface of the heat sources is somehow shielded against the incoming horizontal flow by the vortices located in the upstream direction (for this reason plumes located further downstream have more time to develop along the vertical direction).

At the highest considered value of the Rayleigh number, interestingly, for $\eta=2$ and $\eta=3$ the continuous mechanism of plume removal and regeneration generally affecting the first heat source station is taken over by an alternate phenomenon featuring the emergence of a localized recirculation with a relatively large scale (involving at least two neighboring heat sources). This circulation can temporarily entrap heat, thereby causing a local increase in the temperature, until it breaks and releases heat to the external environment in the form of a localized 'eruption', i.e. a short-lived vigorous vertical movement. These observations lead to a remarkable conclusion. The classical concept of sheltering mechanism (i.e. an increased deflection of the leading buoyant jet with respect to the trailing ones) might be properly 'extended' to highly unsteady (turbulent) dynamics to indicate a variety of new phenomena which somehow still make peculiar the behavior of the first station with respect to the other heat sources located downstream. An exciting prospect 
for the future is to expand the range of applicability of these arguments to real $3 \mathrm{D}$ configurations and/or to implement turbulence models more sophisticated with respect to that used for the present analysis.

\section{APPENDIX}

\section{A The eddy viscosity and thermal diffusivity}

As outlined in Sect. 2.2, with the LES the set of balance equations is parametrized in terms of a spatial operator known as "filter width", which defines the smallest scale under which the turbulent flow behavior must be expressly 'modelled' rather than computed. Accordingly, the resulting approach can capture a variety of scales, which range from the smallest physically relevant scale when the filter width tends to zero (the space-averaged equations tend to original flow equations) to the 'mean' large-scale turbulent flow when a very large filter width is assumed. With the classical Smagorinsky model (Smagorinsky ${ }^{86}$ ) the filter width is set approximately equal to the size of the numerical grid, which allows the eddy viscosity to respond to the existence and the strength of local shears, while retaining a structure that is "consistent with the existence of an inertial range surrounding the grid scale" (Wong and Lilly ${ }^{63}$ ).

By denoting by $v$ and $\alpha$ the kinematic viscosity and thermal diffusivity of the considered fluid, respectively (their ratio being the well-known Prandtl number $\operatorname{Pr}=v / \alpha$ ), with the Smagorinsky model, such quantities must be 'increased' by adding to them the corresponding turbulent counterparts, namely the so-called eddy (or subgrid-scale) viscosity and thermal diffusivity ( $v_{\mathrm{T}}$ and $\alpha_{\mathrm{T}}$ ), respectively.

Such quantities, in turn, depend on the local conditions established in the flow via the relationships:

$$
v_{T}=\tilde{\lambda}^{2} \sqrt{2 \widetilde{S}^{2}}
$$

where $\widetilde{S}$ is the so-called resolved strain rate (in dimensional form), which for a two-dimensional flow having velocity components $u$ and $v$ along $\mathrm{x}$ and $\mathrm{y}$, respectively, reads:

$$
\tilde{S}=\frac{1}{2}\left(\frac{\partial \tilde{u}}{\partial \tilde{y}}+\frac{\partial \tilde{v}}{\partial \tilde{x}}\right)
$$

(where the tilde is used to indicate dimensional quantities) and

$$
\tilde{\lambda}=C_{s} \sqrt{\Delta x \Delta y}
$$

\section{Moreover,}


$\alpha_{T}=\frac{v_{T}}{\operatorname{Pr}_{T}}$

where $\operatorname{Pr}_{\mathrm{T}}$ is known as the turbulent (or subgrid) Prandtl number.

Typically both the non-dimensional coefficient Cs and the subgrid-scale Prandtl number are approximated to be constants. The ranges of values of $C$ s and $\operatorname{Pr}_{\mathrm{T}}$ commonly used are $0.065 \leq \mathrm{Cs} \leq 0.2$ (Li and $\mathrm{Ma}^{71}$ ) and $0.4 \leq \operatorname{Pr}_{\mathrm{T}} \leq 1$ (Edison ${ }^{54}$; Kimmel and Domaradzki ${ }^{64}$ ).

The above practice has its theoretical foundation in the attempt to account for the sub-grid scales removed by the filtering operation by introducing a sub-grid-scale stress tensor and relating its deviatoric (trace-free) part to the resolved strain-rate tensor via $v_{\mathrm{T}}$. Similarly, the sub-grid-scale heat flux is related to the large-scale temperature gradient via $\alpha_{\mathrm{T}}$.

As with this approximation it is implicitly assumed that small thermal scales depend only on the resolved dynamic scales, such a model is in general 'corrected' for the case of natural convection where the flow is obviously produced by buoyant forces and not via a purely dynamic forcing (Salat et al., ${ }^{127}$ ). This idea was originally developed by Lilly ${ }^{87}$ and then further elaborated over the years by other researchers. Both $v_{\mathrm{T}}$ and $\alpha_{\mathrm{T}}$ must be revised to include a terms that takes into account mixing due to statistically unstable conditions (Mason ${ }^{128}$ ). Typically, such corrections are implemented as:

$$
\begin{aligned}
& v_{T}=\tilde{\lambda}^{2} \sqrt{2 \tilde{S}^{2}-g \beta_{T} \frac{\partial \tilde{T}}{\partial \tilde{y}}} \\
& \alpha_{T}=\frac{\tilde{\lambda}^{2}}{\operatorname{Pr}_{T}} \sqrt{2 \tilde{S}^{2}-g \beta_{T} \frac{\partial \tilde{T}}{\partial \tilde{y}}}
\end{aligned}
$$

where $\mathrm{g}$ is the gravity acceleration and $\beta_{\mathrm{T}}$ the fluid thermal expansion coefficient (Wong and Lilly ${ }^{63}$; Kimmel and Domaradzki ${ }^{64}$; Devenish et al., ${ }^{67}$ ). As observed, e.g., by Bastiaans et al., ${ }^{94}$, this is equivalent to assuming a sub-grid model able to parameterize the sub-grid stresses and sub-grid heat fluxes so that, the large flow scale can be accurately computed. The second (buoyancy production) term appearing inside the square root is generally less than, but comparable to the strain term ${ }^{51}$. With the present implementation the overall quantity inside the square root has been constrained as indicated by Eidson ${ }^{54}$ and Kimmel and Domaradzki ${ }^{64}$.

\section{B Numerical method (Projection method)}

In this section we first lay the general foundations of our numerical treatment of the Navier-Stokes equations cast in the framework of the LES model described in Sects. 2.3. In that section, some effort has been devoted to show that, in terms of an appropriate hydrodynamic description of the behavior of the flow on the small scales, the mathematical model can considerably be simplified (no 
need to solve additional equations as it is the case, e.g., of RANS models). The preceding statement, however, should not be misread as implying that the resulting numerical problem is innocuous and free of drawbacks. The numerical solution of the Navier-Stokes equations in the context of the Boussinesq approximation (density variations allowed only in the buoyancy production term) brings problems of its own, including (but not limited) to the issue of making the velocity field effectively divergence free (incompressible). This objective can be pursued by means of various strategies. As a common feature, many of them rely on the natural coupling between velocity and pressure set by the momentum balance equation. These techniques represent a class of methods generally known as primitive-variables methods, to distinguish them from other approaches where the original set of equations and related unknowns are replaced by alternate forms obtained by integration or derivation (e.g., application of the curl operator to the momentum equation to turn it into the balance equation for vorticity, see, e.g., Bucchignani and Stella ${ }^{129-130}$ ).

With the techniques based on primitive variables, initially the dependence on one of the unknowns, that is the pressure, is neglected in the momentum equation. The other primitive variable, namely, the velocity, obtained by integration of the truncated equation has, therefore, scarce physical meaning (the velocity field obtained in this stage is generally called "provisional" or "intermediate" field just to emphasize this concept).

$\underline{V}^{*}=\underline{V}^{n}+\Delta t\left\{-\underline{\nabla} \cdot[\underline{V} \underline{V}]+\left[\frac{1}{\operatorname{Re}}+\lambda^{2} \sqrt{2 S^{2}-\frac{R a}{\operatorname{PrRe}^{2}} \frac{\partial T}{\partial y}}\right] \nabla^{2} \underline{V}-\frac{R a}{\operatorname{Pr}^{2} \operatorname{Re}^{2}} T \underline{i}_{g}\right\}^{n}$

It does not satisfy the incompressibility constraint and does not even take into account the effect of pressure gradients. Nevertheless, it retains an important feature, that is, its 'vorticity' is the same that would be possessed by the equivalent physical velocity. Under a purely mathematical perspective, this property can be seen as the consequence of a well-known concept in vector analysis, i.e. that the application of the curl operator to the gradient of a scalar quantity (the pressure in this case) is zero (vorticity does not depend directly on the pressure gradient). Notably, from an algorithmic point of view, this property is extremely important as it paves the way to the implementation of another very important notion in mathematics, that is, the inverse theorem of vector calculus. Originally used by Ladyzhenskaya ${ }^{131}$ for her studies on the existence and uniqueness of the solutions of the Navier-Stokes equations, this theorem implies the remarkable possibility to make a velocity field unique provided its curl (vorticity) and divergence (and normal component at the boundary) are assigned or known. Following this argument, the intermediate velocity field can formally be expressed as the combination of a solenoidal (divergence-free) component and the gradient of a scalar field (a curl-free part):

$\underline{V}^{*}=\underline{V}+\underline{\nabla} \Phi$ 
If the scalar field is assumed to be proportional to the pressure $\Phi=\xi$ p (where $\xi$ is a constant), the provisional velocity field can formally be expressed as:

$\underline{V^{*}}=\underline{V}+\xi \underline{\nabla} p$

As $\underline{V}$ must be solenoidal, one may also write:

$$
\nabla^{2} p=\frac{1}{\xi} \underline{\nabla} \cdot \underline{V}^{*}
$$

In practice, the possibility to express $\underline{V}$ as a linear combination of $\underline{V}^{*}$ and $\underline{\nabla} p$ and the additional equation (B3) can be used to devise a time-marching procedure for the integration of the original set of Navier-Stokes equations in which viscous and incompressibility effects are accounted for in a segregated manner ${ }^{132-134}$. The initial approximation to the momentum equation (namely eq. (B1)) is initially advanced to determine $\underline{V}^{*}$, then eq. (B3) is solved that enforces the divergence constraint, and finally the physical velocity field is determined via eq. (B2) as $\underline{V}=\underline{V}^{*}-\xi \underline{\nabla} p$, which explains why these techniques are also known as projection methods, fractional-step methods or pressurecorrection methods. This approach was referred to as the Consistent Pressure Poisson Equation (CPPE) in Gresho and Sani ${ }^{135}$. For problem closure $\xi$ must be set equal to $\Delta$ t, that is, the timeintegration step assumed for the time-marching algorithm. The consistency of the approach can finally be verified by applying the curl operator to the divergence-free velocity field:

$\underline{\nabla} \wedge \underline{V}=\underline{\nabla} \wedge \underline{V}^{*}-\Delta t \underline{\nabla} \wedge \underline{\nabla} p=\underline{\nabla} \wedge \underline{V}^{*}$

As the curl of $\underline{V}$ is the same that would be obtained if the momentum equations were solved in its complete form (including the pressure gradient) and its divergence satisfies the continuity equation $\underline{\nabla} \cdot \underline{V}=0$, the obtained velocity field is unique from both mathematical and physical points of view. These techniques have enjoyed a widespread use in the literature especially for categories of flows intrinsically unsteady for which they enable cost-effective solutions by virtue of recent progresses in the capacity of computers and the increasing success of direct numerical simulation in capturing bifurcations and instability phenomena ${ }^{136,137}$. Additional information on this class of methods can be found in Refs [138-142], while for a review of recent advances with regard to their application to situations in which the density cannot be considered constant (Boussinesq approximation no longer valid), the reader may consider Refs $[137,120]$ and references therein.

In the present work, this method has been implemented using schemes explicit in time for the integration of both the momentum and energy equations. Convective terms have been treated using the QUICK scheme while standard central differences have been used to discretize the diffusive terms. Given the delicate role played by the coupling between pressure and velocity (see, e.g., Choi et al., ${ }^{143,144}$ ) a staggered arrangement has been implemented for the primitive variables, that is, 
while pressure occupies the center of each computational cells, the components of velocity $u$ and $v$ are located at the center of the cell face perpendicular to the $\mathrm{x}$ and $\mathrm{y}$ axes, respectively (see, e.g., Lappa $^{145}$ ). The ability of this algorithm to capture adequately the turbulent states of RB convection and related properties was demonstrated in a preceding work of the present authors ${ }^{50}$, which stands as a validation of the present simulations based on the DNS approach.

\section{$\underline{\text { C Boundary conditions }}$}

Even though some relevant information about the boundary conditions (BCs) which make the related IBVP (Initial Boundary Value problem) well posed from a mathematical point view is available (see, e.g., Gresho ${ }^{146}$; and Lappa ${ }^{147}$ ), the identification of proper BCs for open boundaries, however, is not as straightforward as one would imagine. Such boundaries emerge as a consequence of the need to truncate the (otherwise infinite) computational domain in order to make the problem tractable, i.e. representable with a finite number of grid points. Obviously, this modus operandi implicitly requires the introduction of additional numerical BCs. In practice, these are needed to drive the fluid into the domain with desired velocity intensity and direction (consistent with the flow that would have occurred considering an infinitely extended domain), allowing at the same time it to leave the domain without being disturbed by the presence of the boundary. The choice of these BCs has critically been discussed in the literature for a relatively long time and the selection of conditions which make the simulations numerically stable is still being debated ${ }^{148-155}$. These studies have led to a common framework nowadays known as the open boundary condition (OBC) problem. For the present problem, we have found the so-called "prognostic equation" for the outflow (OOBC) (right and top boundaries of the computational domain shown in Fig. 1) to provide reasonable algorithm stability. In the most general form it reads:

$$
\frac{\partial \phi}{\partial t}+C \frac{\partial \phi}{\partial n}+\frac{\partial}{\partial n}\left(D \frac{\partial \phi}{\partial n}\right)=0
$$

where $\phi$ is a generic thermofluid-dynamic quantity, i.e. T, u or v, $C$ is a constant assumed to be equal to the averaged velocity perpendicular to the boundary $C=\underline{V} \cdot \underline{\hat{n}}$ and $D$ is a diffusion coefficient, which can be either the kinematic viscosity for velocity or the thermal diffusivity for temperature. For $C=D=0$, eq. (C1) would reduce to a standard Dirichlet condition, which, however, is unsuitable as it would give rise to a boundary layer and ensuing unphysical results. Similarly, the popular Neumann condition $\frac{\partial \phi}{\partial n}=0$ should also be avoided as, in the case of unsteady flows, it could jeopardize the physical consistency of the solution by causing non-physical fluctuations in the pressure field upstream of the OOBC (reflecting towards the interior at the outflow boundary thereby making the algorithm prone to numerical instability). 
Though the inclusion of the diffusive term appearing in eq. (C1) is known to have beneficial effects on the stability of the time-marching procedure described in APPENDIX B (see, e.g., Stevens ${ }^{148}$ and Fregni et al., ${ }^{9}$ ), it has not been included in the present calculations as our simulations proved to be quite stable over the considered range of conditions by simply discretizing the Robin-type condition represented by the first two terms of eq. (C1) with a leap-frog scheme and an upwind space, respectively, that is:

$\phi_{b}^{n+1}=\left(\frac{1-r}{1+r}\right) \phi_{b}^{n-1}+\left(\frac{2 r}{1+r}\right) \phi_{b-1}^{n}$

where the superscript $\mathrm{n}$ denotes the time step, the subscript $\mathrm{b}$ obviously refers to the boundary cells and $\mathrm{r}=C(\Delta \mathrm{t} / \Delta \mathrm{x})$.

Moreover, we have imposed homogeneous Neumann (zero-gradient) conditions for the pressure on the bottom and left (inflow) boundary, whereas pressure has been set to zero at the open boundary to make the problem well-posed (this condition allows fluid to enter or leave the computational domain if the global divergence of the velocity field is not zero, which is consistent with the behavior of unconfined domains, Craske and van Reeuwijk ${ }^{153}$ ). Additional care, however, has been required to treat the problem relating to vortices leaving the domain through the right or top open boundary. Indeed, vortices implicitly give rise to reversed flow regions as they cross the open boundary, which may give rise to numerical instability if not addressed adequately. In order to do so, a specific procedure was implemented in our algorithm to switch from the conditions represented by eq. (C1) (when the fluid flows out of the domain) to 'backflow conditions' specified to account for flow reversal (fluid flowing into the domain through the open boundary). More specifically, the homogeneous Dirichlet condition for pressure has been locally replaced by the Neumann one, vice versa, for temperature, eq. (C1) has been replaced by a Dirichlet condition with $\mathrm{T}$ set equal to the average temperature along the considered portion of boundary.

\section{References}

[01] Valentine G.A., Wohletz K.H., (1989), Numerical models of Plinian eruption columns and pyroclastic flows, J. Geophys. Res. 94(B2), 1867-1887.

[02] Kaminski E., Tait S. and Carazzo G., (2005), Turbulent entrainment in jets with arbitrary buoyancy, J. Fluid Mech., 526, 361-376.

[03] McGrattan K.B., Baum H.R., Rehm R.G., (1998), Large eddy simulations of smoke movement, Fire Saf. J. 30, 161-178.

[04] Jirka G.H., (2004), Integral Model for Turbulent Buoyant Jets in Unbounded Stratified Flows. Part I: Single Round Jet, Environmental Fluid Mechanics 4: 1-56, 2004.

[5] Tomàs A.F., Poje A.C., Özgökmen T.M., and Dewar W.K., (2016), Dynamics of multiphase turbulent plumes with hybrid buoyancy sources in stratified environments, Phys Fluids, 28, 095109 (24 pp).

[06] Oliver C. J. Davidson M. J. and Nokes R. I., (2013), Removing the boundary influence on negatively buoyant jets, Environmental Fluid Mechanics, 13(6), 625-648 
[07] Martineau R.C., Berry R.A., Esteve A., Hamman K.D., Knoll D.A., Park R., Taitano W., (2010), Comparison of natural convection flows under VHTR type conditions modeled by both the conservation and incompressible forms of the Navier-Stokes equations, Nucl. Eng. Des. 240, 13711385.

[08] Lee J. H.W. and Asce F., (2012), Mixing of Multiple Buoyant Jets, J. Hydraul. Eng., 138(12), 1008-1021.

[09] Fregni A., Angeli D., Cimarelli A., Stalio E., (2019), Direct Numerical Simulation of a buoyant triple jet at low-Prandtl number, Int. J. Heat Mass Transfer, 143, 118466.

[10] Sun Z. and Jaluria Y., (2011), Conjugate thermal transport in gas flow in long rectangular microchannel, J. Electron. Packag. 133(2), 021008 (11 pages).

[11] Biswas N., Mahapatra P. S., Manna N.K. and Roy P.C., (2015), Influence of Heater Aspect Ratio on Natural Convection in a Rectangular Enclosure, Heat Transfer Engineering, 37(2), 125139.

[12] Isaac K.M. and Jakubouski A. K., (1985), Experimental study of the interaction of multiple jets with a cross flow, AIAA Journal, 23(11), 1679-1683.

[13] Baltasar J., Carvalho M.G., Coelho P., Costa M., (1997), Flue gas recirculation in a gas-fired laboratory furnace: measurements and modelling, Fuel , 76(10), 919-929.

[14] Viskanta R., (1993), Heat transfer to impinging isothermal gas and flame jets, Experimental Thermal and Fluid Science, 6(2), 111-134.

[15] Venkatasubbaiah K. and Jaluria Y., (2012), Numerical simulation of enclosure fires with horizontal vents, Numer. Heat Tran. 62, 179-196.

[16] Subudhi S., Sreenivas K.R., Arakeri J.H., (2013), Study of buoyant jets in natural ventilation of a model room, Int. J. Heat Mass Transf. 64, 91-97

[17] Morsli S., Ramenah H., El Ganaoui M. and Bennacer R., (2018), Effect of aligned and misaligned ventilation opening affecting energy demand and air quality in buildings, Eur. Phys. J. Appl. Phys. 83, 10901 (10 pages). https://doi.org/10.1051/epjap/2018180119

[18] Morsli S., Boussoufi M., Sabeur A., El Ganaoui M., Bennacer R., (2018) Small to large scale mixed turbulent convection: buildings application, International Journal of Numerical Methods for Heat \& Fluid Flow, 28(1), 188-205, https://doi.org/10.1108/HFF-10-2017-0392

[19] Harish R., (2018), Effect of heat source aspect ratio on turbulent thermal stratification in a naturally ventilated enclosure, Building and Environment, 143, 473-486.

[20] Smith S. H. and Mungal M. G., (1998), Mixing, structure and scaling of the jet in crossflow, $J$. Fluid Mech., 357: 83-122.

[21] Keramaris E. and Pechlivanidis G., (2016), The Behaviour of a Turbulent Buoyant Jet into Flowing Environment, Procedia Engineering 162, 120 - 127.

[22] Wright S. J., (1977), Mean behavior of buoyant jets in a cross-flow, J. Hydraul. Div., Am. Soc. Civ. Eng., 1035 , 499-513.

[23] Fischer, H. B., List, E. J., Koh, R. C. Y., Imberger, J., and Brooks, N. H. (1979), Mixing in inland and coastal waters, Academic, New York.

[24] Jirka G.H. and Fong H.L.M., (1981), Vortex dynamics and bifurcation of buoyant jets in crossflow, J. Engin. Mech. Div., ASCE 107, EM6.

[25] Kaye N. B. and Linden P. F., (2004), Coalescing axisymmetric turbulent plumes, J. Fluid Mech., 502, 41-63.

[26] Lai, A. C. H., and Lee, J. H.W. (2010), Multiple tandem jet interaction in a crossflow, J. Hydrodyn. Ser. B, 22(5), 639-643.

[27] Lai, A. C. H. and Lee, J. H. W., (2012), Dynamic interaction of multiple buoyant jets, J. Fluid Mech., 708, 539-575.

[28] Lee J. H. W. and Chu V. H., (2003), Turbulent Jets and Plumes: a Lagrangian, Approach. Kluwer, Boston.

[29] Isaac K.M. and Schetz J.A., (1982), Analysis of multiple jets in a cross flow, Journal of Fluids Engineering, 104(4): 489-492. 
[30] Frick W.E., (1984), Non-empirical closure of the plume equations, Atmos. Environ. 18, 653662.

[31] Lee J.H.W. and Cheung V., (1990), Generalized Lagrangian model for buoyant jets in current, J. Environ. Engin. 116, 1085-1106.

[32] Wood, I.R., Bell, R.G. and Wilkinson, D.L.: 1993, Ocean Disposal of Wastewater, World Scientific Publishers, Singapore.

[33] Chu V. H., and Lee J. H. W., (1996), A general integral formulation of turbulent buoyant jets in crossflow, J. Hydraul. Eng., 122(1), 27-34.

[34] Chu P. C. K., Lee J. H.W., and Chu, V. H., (1999). Spreading of turbulent round jet in coflow, J. Hydraul. Eng., 125(2), 193-204.

[35] Morton B.R., Taylor G. I. and Turner J. S., (1956), Turbulent gravitational convection from maintained and instantaneous sources, Proc. R. Soc. Lond. A 234, 1-23.

[36] Sparrow E.M and Minkowycz W. J., (1962), Buoyancy effects on horizontal boundary-layer flow and heat transfer, Int. J. Heat Mass Transfer, 5: 505-511.

[37] Hauptmann E. G., (1965), Laminar boundary-layer flows with small buoyancy effects, Int. J. Heat Mass Transfer, 8, 289-295.

[38] Hieber C. A. (1973), Mixed convection above a heated horizontal surface, Int. J. Heat Mass Transfer, 16, 769-785.

[39] Hussain T. (1988), Mixed convection boundary layer flow on a horizontal plate in a uniform stream, Int. J. Heat Mass Transfer 31(12), 2505-2516.

[40] Qiu X.-L., Yao S. H., and Tong P., (2000), Large-scale coherent rotation and oscillation in turbulent thermal convection, Phys. Rev. E 61(6), R6075-R6078

[41] Qiu X.-L. and Tong P., (2001), Large-scale velocity structures in turbulent thermal convection, Phys. Rev. E 64(3), 036304 [13 pages]

[42] Kadanoff L.P., (2001), Turbulent Heat Flow: Structures and Scaling, Physics Today, 54 (8): 34-39.

[43] Xia, K.-Q., Sun, C. and Zhou, S. Q., (2003) Particle image velocimetry measurement of the velocity field in turbulent thermal convection, Phys. Rev. E, 68, 066303 [18 pages].

[44] Niemela J. J. and Sreenivasan K. R., (2003), Rayleigh-number evolution of large-scale coherent motion in turbulent convection, Europhys. Lett., 62 (6): 829-833.

[45] Lappa M., (2009), Thermal Convection: Patterns, Evolution and Stability, John Wiley \& Sons, Ltd (2009, Chichester, England).

[46] Liou T.M., Hwang J.J. and Chen S.H. (1993), 'Simulation and measurement of enhanced turbulent heat transfer in a channel with periodic ribs on one principal wall', Int. J. Heat Mass Transfer, 36(2), 507-517.

[47] Miyake Y., Tsujimoto K., Nakaji M., (2001), Direct numerical simulation of rough-wall heat transfer in a turbulent channel flow, Int. J. Heat Fluid Flow 22, 237-244.

[48] Miyake, Y., Tsujimoto, K., Nagai, N., (2002), Numerical simulation of channel flow with a rib-roughened wall. J. Turbul. 3, 035.

[49] Chandra P.R., Alexander C.R., Han J.C., (2003), Heat transfer and friction behaviors in rectangular channels with varying number of ribbed walls, Int. J. Heat Mass Transfer 46, 481-495.

[50] Peng W., Jiang P.X., Wang Y.P., Wei B.Y., (2011), Experimental and numerical investigation of convection heat transfer in channels with different types of ribs, Applied Thermal Engineering, 31, 2702-2708.

[51] Fouladi F., Henshaw P., Ting D.S.-K., Ray S., (2017), Flat plate convection heat transfer enhancement via a square rib, Int. J. Heat Mass Transfer, 104, 1202-1216.

[52] Molochnikov V. M., Mazo A. B., Kalinin E. I., Malyukov A. V., Okhotnikov D. I., and Dushina O. A., (2019), Formation and turbulent breakdown of large-scale vortical structures behind an obstacle in a channel at moderate Reynolds numbers, Phys. Fluids 31, 104104 (11 pp). 
[53] Lappa M., (2011), Some considerations about the symmetry and evolution of chaotic Rayleigh-Bénard convection: The flywheel mechanism and the "wind" of turbulence, Comptes Rendus Mécanique, 339, 563-572.

[54] Eidson T.M., (1985), Numerical simulation of turbulent Rayleigh-Bénard convection using subgrid scale modelling, J. Fluid Mech. 158, 245-268.

[55] Yu T.S. and Li C.W., (1998), Instantaneous discharge of buoyant fluid in cross-flow, J. Hydr. Engrg., 124 (11), 1161-1176.

[56] Ma F.X. and Li C.W., (2001), 3D numerical simulation of ambient discharge of buoyant water, Appl. Numer. Model. 25 (5): 375-384.

[57] Xiao Y.. Tang H.W.. Liang D.F.. Zhang J.D., (2011), Numerical study of hydrodynamics of multiple tandem jets in cross flow, Journal of Hydrodynamics, Ser. B, 2(6), 806-813.

[58] Harish R., Venkatasubbaiah K., (2013), Numerical simulation of turbulent plume spread in ceiling vented enclosure, European Journal of Mechanics B/Fluids, 42,142-158.

[59] Harish R. and Venkatasubbaiah K.V, (2016), Non-Boussinesq approach for turbulent buoyant flows in enclosure with horizontal vent and forced inlet port, Applied Mathematical Model. 40, 927-941.

[60] Tsai W. B, Lin W. W, Chieng C. C., (2000), Computation of Enhanced Turbulent Heat Transfer in a Channel with Periodic Ribs, Int. J. of Numerical Methods for Heat and Fluid Flow, 10, $1,47-66$

[61] Weihing P., Younis B.A., Weigand B., (2014), Heat transfer enhancement in a ribbed channel: Development of turbulence closures, Int. J. Heat Mass Transfer 76, 509-522

[62] Lou Y., He Z., Jiang H., and Han X., (2019), Numerical simulation of two coalescing turbulent forced plumes in linearly stratified fluids, Phys Fluids, 31, 037111 (16 pp).

[63] Wong V. C. and Lilly D. K., (1994), A comparison of two dynamic subgrid closure methods for turbulent thermal convection, Phys. Fluids 6, 1016-1023.

[64] Kimmel S.J. and Domaradzki J. A., (2000), Large eddy simulations of Rayleigh-Bénard convection using subgrid scale estimation model, Phys. Fluids 12, 169-184.

[65] Pham M. V., Plourde F. and Doan K.S., (2007), Direct and large-eddy simulations of a pure thermal plume, Phys. Fluids, 19, 125103.

[66] Yan Z. H., (2007), Large-eddy simulations of a turbulent thermal plume, Heat Mass Transfer $43,503-514$.

[67] Devenish B., Rooney G., Thomson D., (2010), Large-eddy simulation of a buoyant plume in uniform and stably stratified environments, J Fluid Mech., 652,75-103.

[68] Pant C. S., Bhattachary A., (2016), A viscous sponge layer formulation for robust large eddy simulation of thermal plumes, Computers and Fluids 134-135, 177-189

[69] Morton B.R., Nguyen K.C., Cresswell R.W., (1994), Similarity and self-similarity in the motion of thermals and puffs, Recent Research Advances in the Fluid Mechanics of Turbulent Jets and Plumes, 1994, pp. 89-116.

[70] Li C.W. and Wang J.H.,(2000), Large eddy simulation of free surface shallow-water flow, Int. J. Numer. Meth. Fluids 34, 31-46.

[71] Li C.W.and Ma F.X., (2003), Large eddy simulation of diffusion of a buoyancy source in ambient water, Applied Mathematical Modelling 27, 649-663.

[72] Ciofalo M. and Collins M.W., (1992), Large-eddy simulation of turbulent flow and heat transfer in plane and rib-roughened channels, Int. J. Numer. Methods Fluids 15 (4), 453-489.

[73] Murataa A., Mochizuki S., (2000), Large eddy simulation with a dynamic subgrid-scale model of turbulent heat transfer in an orthogonally rotating rectangular duct with transverse rib turbulators, Int. J. Heat Mass Transfer 43 (7), 1243- 1259.

[74] Cui J., Patel V.C., Lin C., (2003), Large-eddy simulation of turbulent flow in a channel with rib roughness, Int. J. Heat Fluid Flow 24, 372-388.

[75] Lohász MM, Rambaud P, Benocci C., (2006), Flow features in a fully developed ribbed duct flow as a result of MILES, Flow Turbulence Combust, 77:59-76. 
[76] Labbé O., (2013), Large-eddy-simulation of flow and heat transfer in a ribbed duct, Computers \& Fluids 76,23-32

[77] Kolmogorov, A.N. (1941a) The local structure of turbulence in incompressible viscous fluids at very large Reynolds numbers, Dokl. Akad. Nauk. SSSR 30, 299-303. Reprinted in Proc. R. Soc. London A 434, 9-13 (1991).

[78] Kolmogorov, A.N. (1941b) On the degeneration of isotropic turbulence in an incompressible viscous fluids, Dokl. Akad. Nauk. SSSR 31, 538-541.

[79] Kolmogorov, A.N. (1941c) Dissipation of energy in isotropic turbulence. Dokl. Akad. Nauk. SSSR, 32, 19-21.

[80] Kolmogorov, A.N. (1942) Equations of turbulent motion in an incompressible fluid. Izv. Akad. Nauk. SSSR ser. Fiz. 6, 56-58.

[81] Bhattacharjee J.K., (2015), Kolmogorov argument for the scaling of the energy spectrum in a stratified fluid, Physics Letters A, 379, 696-699.

[82] Kraichnan R.H., (1974), On Kolmogorov's inertial-range theories, J. Fluid Mech., 62, 305-330

[83] Bolgiano R., (1959), Turbulent spectra in a stably stratified atmosphere, J. Geophys. Res. 64, 2226-2229.

[84] Obukhov A.M., (1959), On the influence of Archimedean forces on the structure of the Temperature field in a turbulent flow, Dokl. Akad. Nauk SSSR, 125, 1246-1248

[85] Kumar A., Chatterjee A.G., and Verma M.K., (2014), Energy spectrum of buoyancy-driven turbulence, Phys. Rev. E 90, 023016

[86] Smagorinsky J., (1963), General circulation experiments with the primitive equations. Part I: the basic experiment, Monthly Weather Rev. 91, 91-152.

[87] Lilly D. K., (1962), On the numerical simulation of buoyant convection, Tellus 15, 148-172.

[88] Dunn W.E., Policastro A.J., Paddock R.A., (1975), Surface Thermal Plumes: Evaluation of Mathematical Models for the Near and Complete Field, Report No. ANL/WR-75-3, Argonne National Laboratory, Argonne, Illinois, August 1975.

[89] Vincent A.P. and Yuen D.A., (1999), Plumes and waves in two-dimensional turbulent thermal convection, Phys. Rev. E 60(3), 2957-2963.

[90] Hier Majumder C. A., Yuen D. A. and Vincent A., (2004), Four dynamical regimes for a starting plume model, Phys. Fluids, 16(5), 1516-1531.

[91] Georgiadis N.J., Rizzetta D.P. and Fureby C., (2010), Large-Eddy Simulation: Current Capabilities, Recommended Practices, and Future Research, AIAA Journal, 48(8): 1772-1784.

[92] Choi H. and Moin P., (2012), Grid-point requirements for large eddy simulation: Chapman's estimates revisited, Phys. Fluids, 24, 011702.

[93] Farhangnia M., Biringen S, Peltier L. J., (1996), Numerical Simulation of Two-dimensional Buoyancy-driven Turbulence in a Tall Rectangular Cavity, Int. J. Numer. Meth. Fluids, 23(12): $1311-1326$.

[94] Bastiaans R. J.M., Rindt C. C. M., Nieuwstadt F. T. M. and van Steenhoven A. A., (2000), Direct and large-eddy simulation of the transition of two- and three-dimensional plane plumes in a confined enclosure, Int. J. Heat Mass Transfer 43, 2375-2393.

[95] Coceal O., Thomas T.G., Castro I.P. , Belcher S. E., (2006), Mean flow and turbulence statistics over groups of urban-like cubical obstacles, Boundary-Layer Meteorol., 121, 491-519

[96] Paolucci S., (1990), Direct numerical simulation of two-dimensional turbulent natural convection in an enclosed cavity, J. Fluid Mech., 215, 229-262.

[97] Kerr R.M., (1996), Rayleigh number scaling in numerical convection, J. Fluid Mech. 310, 139179.

[98] De A.K., Eswaran V., Mishra P.K., (2017), Scalings of heat transport and energy spectra of turbulent Rayleigh-Bénard convection in a large-aspect-ratio box, Int. J. Heat Fluid Flow, 67, 111124.

[99] Lohse D. and Xia K.Q., (2010), Small-scale properties of turbulent Rayleigh-Bénard convection, Ann. Rev. Fluid Mech., 42, 335-364 . 
[100] Cortese T. and Balachandar S., (1993), Vortical nature of thermal plumes in turbulent convection, Phys. Fluids A, 5, 3226-3232.

[101] Plourde F., Pham M. V., Kim S. D. and Balanchandar S., (2008), Direct numerical simulations of a rapidly expanding thermal plume: structure and entrainment interaction, J. Fluid Mech. 604, 99-123.

[102] Nakayama H. and Nagai H., (2010), Large-eddy simulation on turbulent flow and plume dispersion over a 2-dimensional hill, Adv. Sci. Res., 4, 71-76.

[103] Nakayama H., Takemi T., and Nagai H., (2011), LES analysis of the aerodynamic surface properties for turbulent flows over building arrays with various geometries, J. Appl. Meteorol. Clim., $6,79-86$.

[104] Nakayama H., Takemi T., and Nagai H., (2012), Large-eddy simulation of urban boundarylayer flows by generating turbulent inflows from mesoscale meteorological simulations, Atmos. Sci. Lett., 13, 180-186.

[105] Nakayama H., Jurcakova K., and Nagai H., (2013), Development of local-scale highresolution atmospheric dispersion model using large-eddy simulation Part 3: turbulent flow and plume dispersion in building arrays, J. Nucl. Soc. Technol., 50, 503-519.

[106] Nakayama H., Leitl B., Harms F., Nagai H., (2014a), Development of local-scale highresolution atmospheric dispersion model using large-eddy simulation Part 4: turbulent flows and plume dispersion in an actual urban area, J. Nucl. Soc. Technol., 51, 628-638.

[107] Nakayama H., Takemi T., and Nagai H., (2014b), Large-eddy simulation of plume dispersion under various thermally stratified boundary layers, Adv. Sci. Res., 11, 75-81.

[108] Lappa M., (2018), On the transport, segregation and dispersion of heavy and light particles interacting with rising thermal plumes, Phys. Fluids, 30(3), 033302 (23 pages).

[109] Lappa M., (2003) "Growth and Mutual Interference of Protein Seeds under reduced gravity conditions", Phys. Fluids, 15 (4): 1046-1057.

[110] Lappa M., (2005), "Discrete layers of interacting growing protein seeds: convective and morphological stages of evolution", Phys. Rev. E Statistical Nonlinear, and Soft Matter Physics, 71 (3): 031904 (12 pages).

[111] Lappa M., (2006), Oscillatory convective structures and solutal jets originated from discrete distributions of droplets in organic alloys with a miscibility gap, Phys. Fluids, 18 (4), 042105 (14 pages).

[112] Lappa M., (2007), "Single- and Multi-droplet configurations out of thermodynamic equilibrium: pulsating, traveling and erratic fluid-dynamic instabilities", Chapter 1 (pp. 1-58) in New Colloid and Surface Science Research, Editor Lawrence V. Schwartz, 2007 Nova Science Publishers Inc.

[113] Pera L. and Gebhart B., (1971), On the stability of laminar plumes: Some numerical solutions and experiments, Int. J. Heat Mass Transfer, 14(7), 975-982.

[114] Qiao M., Tian Z.F., Nie B., and Xu F., (2018), The route to chaos for plumes from a top open cylinder heated from underneath, Phys Fluids, 30, 124102 (12 pp).

[115] Desrayaud G. and Lauriat G., (1993), Unsteady Confined Buoyant Plumes, J. Fluid Mech., 252: 617-646.

[116] Vincent A.P. and Yuen D.A., (2000), Transition to turbulent thermal convection beyond $\mathrm{Ra}=10^{10}$ detected in numerical simulations, Phys. Rev. E, 61(5): 5241-5246.

[117] Shao D., Huang D., Jiang B., Law A.W.K., (2017), Flow patterns and mixing characteristics of horizontal buoyant jets at low and moderate Reynolds numbers, Int. J. Heat Mass Transf 105, $831-846$.

[118] Zhou S.Q. and Xia K.Q., (2002), Plume Statistics in Thermal Turbulence: Mixing of an Active Scalar, Phys. Rev. Lett., 89(18), 184502.

[119] Djenidi L., Antonia R.A., Talluru M.K. and Abe H., (2017), Skewness and flatness factors of the longitudinal velocity derivative in wall-bounded flows, Phys. Rev Fluids, 2, 064608. 
[120] Lappa M. and Gradiscak T., (2018), On the Oscillatory Modes of Compressible Thermal Convection in inclined differentially heated cavities, Int. J. of Heat and Mass Transfer, 121: 412436.

[121] Oskouie S.N., Yang Z., and Wang B.C., (2018), Study of passive plume mixing due to two line source emission in isotropic turbulence, Phys Fluids, 30, 075105 (14 pp).

[122] Yu D., Ali M.S. and Lee J. H.W., (2006), Multiple Tandem Jets in Cross-Flow, J. Hydraul. Eng., 2006, 132(9), 971-982

[123] Mason P. J. and Sykes R. I., (1979), Three-dimensional numerical integrations of the NavierStokes equations for flow over surface-mounted obstacles, J. Fluid Mech. 91(3), 433-450

[124] Largeau J.F. and Moriniere V., (2007), Wall pressure fluctuations and topology in separated flows over a forward-facing step, Exp Fluids, 42: 21-40.

[125] Abdalla I. E., Yang Z. and Cook M., (2009), Computational analysis and flow structure of a transitional separated-reattached flow over a surface mounted obstacle and a forward-facing step, International Journal of Computational Fluid Dynamics, 23:1, 25-57, DOI: $10.1080 / 10618560802566246$

[126] Hattori H, Nagano Y., (2010), Investigation of turbulent boundary layer over forward facing step via direct numerical simulation, Int J Heat Fluid Flow, 31:284-294.

[127] Salat J., Xin S., Joubert P., Sergent A., Penot F., Le Quere P., (2004), Experimental and numerical investigation of turbulent natural convection in a large air-filled cavity, Int. J. Heat Fluid Flow, 25: 824-832.

[128] Mason P. J., (1994), Large-Eddy Simulation of the Convective Atmospheric Boundary Layer, J. Atmos. Sci. 46, 1492-1516.

[129] Bucchignani E. and Stella F., (1999a), Rayleigh-Bénard convection in limited domains: Part 1 - Oscillatory flow, Num. Heat Tr. Part A, 36(1), 1-16.

[130] Bucchignani E. and Stella F., (1999b), Rayleigh-Bénard convection in limited domains: Part 2 - transition to chaos, Num. Heat Tr. Part A, 36(1), 17-34.

[131] Ladyzhenskaya O.A., (1969), The Mathematical Theory of Viscous Incompressible Flow, Gordon and Breach, 2nd Edition, New York - London, 1969.

[132] Harlow F.H. and Welch J.E. (1965), Numerical calculation of time-dependent viscous incompressible flow with free surface, Phys. Fluids, 8: 2182-2189.

[133] Temam R., (1968), Une méthode d'approximation de la solution des équations de NavierStokes, Bull. Soc. Math. France, 98: 115-152.

[134] Chorin A.J., (1968), Numerical solutions of the Navier-Stokes equations, Math. Comput., 22: 745-762.

[135] Gresho P.M., Sani R.T., (1987), On pressure boundary conditions for the incompressible Navier-Stokes equations, Int. J. Numer. Meth. Fluids, 7: 1111-1145.

[136] Lappa M., (2019), A mathematical and numerical framework for the simulation of oscillatory buoyancy and Marangoni convection in rectangular cavities with variable cross section, Chapter 12 (pp. 419-458) in Computational Modeling of Bifurcations and Instabilities in Fluid Mechanics (Springer Mathematical Series, 2018, Part of the Computational Methods in Applied Sciences book series - COMPUTMETHODS, volume 50), Editor Alexander Gelfgat - ISBN 978-3-319-91493-0. DOI: $10.1007 / 978-3-319-91494-7$.

[137] Lappa M., (2016), A Mathematical and Numerical Framework for the Analysis of Compressible Thermal Convection in Gases at very high Temperatures, Journal of Computational Physics, 313: 687-712.

[138] Guermond J.-L., (1996), Some practical implementations of projection methods for Navier Stokes equations, Model. Math. Anal. Num., 30: 637-667.

[139] Guermond J.-L. and Quartapelle L., (1998), On stability and convergence of projection methods based on pressure Poisson equation, Int. J. Numer. Meth. Fluids, 26: 1039-1053.

[140] Strikwerda J.C. and Lee Y.S., (1999), The accuracy of the fractional step method, SIAM J. Numer. Anal., 37: 37-47. 
[141] Petersson N.A., (2001), Stability of Pressure Boundary Conditions for Stokes and NavierStokes Equations, J. Comput. Phys., 172: 40-70.

[142] Guermond J.-L., Minev P. and Shen J., (2006), An Overview of Projection Methods for Incompressible Flows. Comput. Methods, Comput. Methods Appl. Mech. Eng., 195: 6011-6045.

[143] Choi S.K., Nam H.Y., Cho M. (1994a), Systematic comparison of finite-volume calculation methods with staggered and nonstaggered grid arrangement, Numer Heat Transfer, Part B 25 (2): 205-221

[144] Choi S.K., Nam H.Y., Cho M., (1994b), Use of staggered and nonstaggered grid arrangements for incompressible flow calculations on nonorthogonal grids, Numer Heat Transfer, Part B 25(2): 193-204.

[145] Lappa M., (1997), Strategies for parallelizing the three-dimensional Navier-Stokes equations on the Cray T3E, Science and Supercomputing at CINECA, 11: 326-340.

[146] Gresho P.M., (1991), Incompressible fluid dynamics: some fundamental formulation issues, Ann. Rev Fluid Mech., 23: 413-453.

[147] Lappa M., (2002), Well-posed problems for the Navier-Stokes equations in the microgravity environment, MSSU: Microgravity \& Space Station Utilization (ISSN: 0958-5036), 3(4), 51-62.

[148] Stevens D.P., (1990), On open boundary conditions for three dimensional primitive equation ocean circulation models, Geophys. Astrophys. Fluid Dyn. 51, 103-133.

[149] Miyauchi T , Tanahashi M, Suzuki M., (1996), Inflow and outflow boundary conditions for direct numerical simulations, JSME Int. J. Ser B, 39(2),305-314 .

[150] Comini G. and Nonino C., (1998), The Outflow Boundary Condition For Mixed Convection Problems, WIT Transactions on Engineering Sciences, 18, 10, DOI 10.2495/AFM980081.

[151] Ol'shanskii M.A. and Staroverov V.M., (2000), On simulation of outflow boundary conditions in finite difference calculations for incompressible fluid, Int. J. Numer. Methods Fluids, 33,499-534.

[152] Craske J. and van Reeuwijk M., (2013), Robust and accurate open boundary conditions for incompressible turbulent jets and plumes, Computers and Fluids, 86, 284-297.

[153] Hattori T , Norris S , Kirkpatrick M , Armfield S., (2013), Comparison of non-reflective boundary conditions for a free-rising turbulent axisymmetric plume, Int. J. Numer. Methods Fluids, 72(12), 1307-1320.

[154] Dong S., Karniadakis G.E., Chryssostomidis C., (2014), A robust and accurate outflow boundary condition for incompressible flow simulations on severely-truncated unbounded domains, J. Comput. Phys., 261, 83-105.

[155] Shekhar Pant C. , Bhattachary A., (2016), A viscous sponge layer formulation for robust large eddy simulation of thermal plumes, Computers and Fluids 134-135, 177-189. 